Copyright by

Eric Dexter Swenson 2006 
The Dissertation Committee for Eric Dexter Swenson certifies that this is the approved version of the following dissertation:

\title{
Efficient Frequency Response Analysis of Structures with Viscoelastic Materials
}

\author{
Committee:
}

Jeffrey K. Bennighof, Supervisor

Ronald O. Stearman

Linda J. Hayes

Robert A. van de Geijn

Clint N. Dawson 


\title{
Efficient Frequency Response Analysis of Structures with Viscoelastic Materials
}

\author{
by
}

Eric Dexter Swenson, B.S.; M.S.

\author{
Dissertation \\ Presented to the Faculty of the Graduate School of \\ The University of Texas at Austin \\ in Partial Fulfillment \\ of the Requirements \\ for the Degree of \\ Doctor of Philosophy
}

The University of Texas at Austin

December 2006 
To my wife and family 


\section{Acknowledgments}

I would like to express my gratitude to Dr. Bennighof for his advice, teaching, and guidance. I have benefited greatly from Dr. Bennighof's expertise, insight, and careful reading of my dissertation.

I would like to express my gratitude to my five other committee members. It has been a great honor to work with Dr. Craig, Dr. Stearman, Dr. Hayes, Dr. van de Geijn, and Dr. Dawson. I appreciate all of their guidance and advice both academic and non-academic.

I am very grateful to Dr. Mintae Kim and Mark Muller who have really helped me throughout the entire process. Our discussions on programming, theory, and mathematics have served as a cornerstone in my development. I would also like to thank Dr. Matthew Kaplan, Dr. Chang-Wan Kim, Jeremiah Palmer, Garrett Moran, and Tim Allison for their hard work on and dedication to AMLS.

I would also like to thank Roush Industries for samples, technical data, and interest in my work. I would also like to thank Mladen Chargin in $\mathrm{CDH} \mathrm{GmbH}$ for his support on this research. 
Finally, and most importantly, I would like to thank my family. I am grateful for my parent's support and interest in my work. To my wife and children, thank you for your unconditional love and support.

ERIC Dexter Swenson

The University of Texas at Austin

December 2006 


\section{Efficient Frequency Response Analysis of Structures with Viscoelastic Materials}

Publication No.

Eric Dexter Swenson, Ph.D.

The University of Texas at Austin, 2006

Supervisor: Jeffrey K. Bennighof

Noise and vibration levels in structures like automobiles and aircraft have been reduced through the application of viscoelastic materials (VEMs) as damping treatments for many years $[18,34,37]$. Adding a VEM to a structure makes accurate prediction of a structure's response to harmonic excitations challenging. This is because the VEM's properties, including the Young's modulus, damping coefficient, and shear modulus, vary significantly as functions of both frequency of excitation and temperature [34]. The solution algorithm presented in this research takes advantage of the fact that the VEM properties typically vary smoothly with frequency by interpolating VEM property variations between known values at perhaps a half dozen frequencies.

The typical finite element (FE) discretization targeted by this work has mil- 
lions of FE degrees of freedom in order to obtain acceptable accuracy over the frequency range of interest and is typically solved at hundreds of frequencies for tens to hundreds of load cases. Accurate approximate solutions to this large frequency response problem (FRP) can be computed efficiently on an approximating subspace. To decrease the cost of factoring the resulting reduced FRP at every frequency, the dimension of the approximating subspace is minimized by replacing hundreds to thousands of eigenvectors with a significantly smaller number of enrichment vectors called residual flexibility vectors (RFVs), damping deformation vectors (DDVs), and dynamic response vectors (DRVs). The RFVs and DDVs represent quasistatic response to loads and to dashpot forces, respectively, and including RFVs and DDVs in the approximating subspace is a common industrial practice.

The use of DRVs, which are corrections to approximate solutions of the FRP at select frequencies, is new. Because computing DRVs is very expensive on the FE subspace, we accurately approximate DRVs in a reduced subspace associated with the automated multilevel substructuring (AMLS) method. Also, we attempt to minimize the number of DRVs by computing those that will improve the accuracy of frequency response solutions the most. Overall, the cost of solving FRPs with VEMs can be reduced dramatically by including DRVs in the approximating subspace, because the accuracy obtained using DRVs could only be achieved otherwise by including a much larger number of global eigenvectors in the approximating subspace. 


\section{Contents}

Acknowledgments $\quad$ v

$\begin{array}{lll}\text { Abstract } & \text { vii }\end{array}$

List of Tables $\quad$ xiv

List of Figures $\quad$ xvi

$\begin{array}{lll}\text { Chapter } 1 & \text { Introduction } & 1\end{array}$

1.1 Frequency Response Problem . . . . . . . . . . . . . . . . 2

1.2 Computational Challenges . . . . . . . . . . . . . . . . 3

1.3 Representing the Frequency Dependence of Damping and Stiffness in

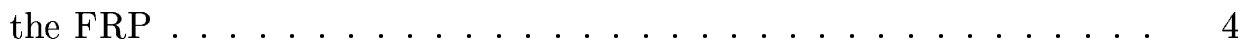

1.4 Solving the Frequency Response Problem . . . . . . . . . . . . . 6

1.5 Outline of Dissertation . . . . . . . . . . . . . . 8

Chapter 2 Properties and Configurations of Viscoelastic Materials 9

2.1 Viscoelastic Material Properties . . . . . . . . . . . . . . . . . . . 9 
2.2 VEM Configuration . . . . . . . . . . . . . . . . 13

2.3 Finite Element Modeling Approaches for Constrained Layer Damping Configuration ........................ 15

2.3.1 Equivalent Shell Approach . . . . . . . . . . . . . . . 17

2.3.2 "Shell/Volume/Shell" Approach . . . . . . . . . . . . 17

Chapter 3 Survey of Frequency Dependent Analysis Methods $\quad 20$

3.1 Representing Frequency Dependence in the FRP . . . . . . . . . . 20

3.1.1 Frequency Independent Properties . . . . . . . . . . . . 21

3.1.2 Frequency Ranges of Constant Properties . . . . . . . . . . 21

3.1.3 Modal Strain Energy Method . . . . . . . . . . . . . 22

3.1.4 Revised MSE Method . . . . . . . . . . . . . . . 24

3.1.5 Internal Variable Methods . . . . . . . . . . . . . 26

3.2 Survey of Previously Developed Solution Techniques . . . . . . . . . 26

3.2 .1 Direct Solution . . . . . . . . . . . . . . 27

3.2 .2 Iterative Solution . . . . . . . . . . . . . . 27

3.2.3 Model Reduction Methods . . . . . . . . . . . . . 28

Chapter 4 New Approach to Solving the FRP with Frequency De$\begin{array}{lr}\text { pendent Materials } & 32\end{array}$

4.1 Interpolation Scheme . . . . . . . . . . . . . . . . . 33

4.2 Approximating Subspace . . . . . . . . . . . . . . . . 37

4.2 .1 Eigenvectors . . . . . . . . . . . . . . 38 
4.2.2 Residual Flexibility Vectors . . . . . . . . . . . . . . . 39

4.2.3 Damping Deformation Vectors . . . . . . . . . . . 39

4.2.4 Dynamic Response Vectors . . . . . . . . . . . . . . . 40

\section{Chapter 5 Computation of the Approximating Subspace 41}

5.1 Partially Constrained Layer Plate Example . . . . . . . . . . . . . . 42

5.2 History of the Automated Multi-Level Substructuring (AMLS) Method 43

5.3 Overview of the AMLS method . . . . . . . . . . . . . 44

5.4 Overview of the Implementation . . . . . . . . . . . . . . 45

5.5 Phase 2: Define Substructure Tree . . . . . . . . . . . . . . 47

5.6 Phase 3: Project System Matrices onto AMLS Subspace . . . . . . . 50

5.7 Phase 4: Generating the Distilled Subspace . . . . . . . . . . . 61

5.7 .1 Computing the Eigenpairs . . . . . . . . . . . . 65

5.7.2 Computing the Residual Flexibility Vectors . . . . . . . . 65

5.7.3 Computing the Damping Deformation Vectors . . . . . . 66

5.7.4 Orthogonalization of the RFVs and DDVs . . . . . . 66

5.8 Phase 4d: Computing the Dynamic Response Vectors _ . . . . . . . 67

5.8.1 Projecting the Frequency Response Problem onto the Distilled Subspace ........................ 67

5.8.2 Computing Dynamic Response Vectors . . . . . . . . . . . 69

5.8.3 Orthogonalization of the Dynamic Response Vectors . . . . 73

5.8.4 Solving the Small Eigenvalue Problem . . . . . . . . . . . . 74 
5.8.5 Computation of Modal Mass Matrix . . . . . . . . . . 74

5.9 Phase 5: Backtransformation to the FE space $\ldots \ldots \ldots 75$

5.10 Phase 6d: Solving the FRP on the Approximating Subspace . . . . . 76

$\begin{array}{lll}\text { Chapter } 6 & \text { Numerical Results } & \mathbf{7 7}\end{array}$

6.1 Parameters ............................ 78

6.2 Cantilever Plate Model . . . . . . . . . . . . . . . . . . . . . . . 79

6.3 Group One Parameters . . . . . . . . . . . . . . . . . . . . 82

6.3.1 Effect of the Nominal Frequency . . . . . . . . . . . . . 82

6.3.2 Effect of the Residual Flexibility Vectors (RFVs) . . . . . . 84

6.3.3 Effect of the Damping Deformation Vectors (DDVs) . . . . 84

6.4 Group Two Parameters . . . . . . . . . . . . . . . . . . 87

6.4.1 Effect of the Global Cutoff Frequency . . . . . . . . . . 87

6.4.2 Effect of the Selection of Dynamic Response Vectors (DRVs) 90

6.4.3 Effect of the Type of Dynamic Response Vectors (DRVs) . . 91

6.4.4 Quarter Panel FE Model . . . . . . . . . . . . . . . . . . . 94

6.4.5 Effect of the Substructure Cutoff Frequency . . . . . . . . . 95

6.4.6 New Approach Applied to Quarter Panel Model . . . . . . . 98

6.5 "Body-in-White" Automobile FE Model . . . . . . . . . . . . . . . . 102

$\begin{array}{lll}\text { Chapter } 7 & \text { Conclusions and Future Work } & 112\end{array}$

7.1 Conclusions . . . . . . . . . . . . . . . . . . . . . . 113

7.2 Future Work . . . . . . . . . . . . . . . . . . 116 
Vita

127

xiii 


\section{List of Tables}

6.1 Effect of the global cutoff frequency $\omega_{g}$ on elapsed time for all steps of the solution algorithm for the cantilever plate model . . . . . . 89

6.2 Effect of the global cutoff frequency $\omega_{g}$ for the cantilever plate model 89

6.3 Effect of including DRVs in the approximating subspace on elapsed time for all steps of the solution algorithm for the cantilever plate model ............................ 92

6.4 Effect of substructure cutoff frequency $\omega_{A}$ on elapsed time for all steps of the solution algorithm for the quarter panel model . . . . . . . . 96

6.5 Effect of substructure cutoff frequency $\omega_{A}$ for the quarter panel model 98

6.6 Comparison of the elapsed time for all steps of the solution algorithm for the quarter panel model . . . . . . . . . . . . . . . . . . 100

6.7 Comparison of the elapsed time for all steps of the solution algorithm for the quarter panel model . . . . . . . . . . . . . . . . . . 100

6.8 Parameters of the solution approach for the quarter panel model . . 101

6.9 Parameters of the solution approach for the "body-in-white" model . 105 
6.10 Comparison of the elapsed time for all steps of the solution algorithm for the "body-in-white" model . . . . . . . . . . . . . . . . . 105

6.11 Effect of the substructure cutoff frequency $\omega_{A}$ on solution parameters for the "body-in-white" model . . . . . . . . . . . . . . . . . . 106

6.12 Effect of the substructure cutoff frequency $\omega_{A}$ on elapsed time for the "body-in-white" model . . . . . . . . . . . . . . . . . . . . . . . 109 


\section{List of Figures}

2.1 Cyclic stress-strain relationship vs time for (a) ideal elastic material (b) ideal viscous material (c) VEM $[29] \ldots \ldots$. . . . . . . . . 10

2.2 Frequency dependence of VEM properties of Young's modulus $E(\omega)$ and loss factor $\eta(\omega)$ for a typical VEM . . . . . . . . . . . . . 11

2.3 CLD product in bending . . . . . . . . . . . . . . . . 13

2.4 (a) Example of a PCL configuration, (b) Example of a CLD configuration ............................ 14

2.5 FE model of CLD products . . . . . . . . . . . . . . . 18

4.1 Example cubic spline interpolation functions for 4 sampling locations: $0,400,800$, and $1200 \mathrm{~Hz} \ldots \ldots \ldots \ldots \ldots$

4.2 Comparison of the interpolated Young's modulus and the actual Young's modulus . . . . . . . . . . . . . . . . . . . 35

4.3 Comparison of the interpolated and the actual damping factors . . . 36 
5.1 (a) Partially constrained layer (PCL) plate example, (b) Example plate partitioned into seven substructures . . . . . . . . . . . 42

5.2 Flowchart of the solution sequence . . . . . . . . . . . . . 46

5.3 Depiction of interior and shared nodes . . . . . . . . . . . . . . 47

5.4 (a) Sparsity pattern $M$ and $K$ after reorder in Phase 2, (b) Substructure tree diagram of $M$ and $K \ldots \ldots \ldots \ldots$

5.5 (a) Sparsity pattern of $K_{j}$ after reorder in Phase 2 (b) Substructure tree diagram of $K_{j} \ldots \ldots \ldots \ldots \ldots \ldots \ldots \ldots$

5.6 Subtree Diagram . . . . . . . . . . . . . . . . . . . 61

5.7 Substructure tree diagram of the distillation process for $K_{j D} \ldots \ldots 68$

6.1 Cantilever plate model . . . . . . . . . . . . . . . . . 79

6.2 RA925 Properties . . . . . . . . . . . . . . . 80

6.3 Effect of the nominal frequency $\omega_{n}$ for the cantilever plate model with inset in bottom graph . . . . . . . . . . . . . . 83

6.4 Effect of including a RFV in the approximating subspace on solution accuracy for the cantilever plate model with inset in bottom graph .

6.5 Effect of including a DDV in the approximating subspace on solution accuracy for the cantilever plate model with inset in bottom graph .

6.6 Effect of the global cutoff frequency $\omega_{g}$ on solution accuracy for the cantilever plate model with inset in bottom graph . . . . . . . 88 
6.7 Values of the energy seminorm ratios of the residuals for the cantilever plate problem $\ldots \ldots \ldots \ldots \ldots$. . . . . . . . . . . . . . 91

6.8 Effect of including DRVs in the approximating subspace on solution accuracy for the cantilever plate model with inset in bottom graph . 93

6.9 (a) FE representation of a automobile quarter panel (b) FE representation of the wheel house . . . . . . . . . . . . . . . 94

6.10 Effect of substructure cutoff frequency $\omega_{A}$ on solution accuracy for the quarter panel model with inset in bottom graph $\ldots . . . .$.

6.11 Comparison of four approximate solutions for the quarter panel model with inset in bottom graph . . . . . . . . . . . . . . 99

6.12 (a) FE representation of a "body-in-white" automobile body (b) FE representation of the dashboard, wheel houses, firewall, floor pan, and trunk well (c) Cross section of constrained layer damping sandwich . 103

6.13 Accuracy of approximate solutions for the "body-in-white" model with inset in bottom graph . . . . . . . . . . . . . . . . 104

6.14 Effect of the substructure cutoff frequency $\omega_{A}$ on solution accuracy for the "body-in-white" model with inset in bottom graph . . . . . . 107

6.15 Comparison of approximate solutions for the "body-in-white" model with inset in bottom graph . . . . . . . . . . . . . 108

6.16 Effect of the number of DRVs on solution accuracy for the "body-inwhite" model with inset in bottom graph . . . . . . . . . . . 111 


\section{Chapter 1}

\section{Introduction}

Noise and vibration in structures like automobiles and aircraft have been effectively reduced through the application of viscoelastic materials (VEMs) as damping treatments for many years $[37,34,18]$. A VEM has both the strain energy storage property of an elastic material and the energy dissipation property of a viscous material. Noise and vibration in these structures are generated from sources such as the engine, power train, pumps, and gears. VEMs, such as plastics, rubbers, acrylics, and epoxies, can be directly sprayed or glued to an existing structural surface, such as a door or roof panel, to damp unwanted vibrations. A more effective damping configuration is to constrain the VEM by adding an additional layer of stiff material like steel or aluminum making a built-up sandwich with a VEM core. This configuration, referred to as the constrained layer damping (CLD) configuration, is more effective because the VEM core dissipates more vibrational energy due to large 
shear strains in the VEM core.

Adding a VEM to a structure makes accurate prediction of a structure's response to harmonic excitations challenging to compute in a reasonable amount of time. The VEM's properties, including the Young's modulus, damping coefficient, and shear modulus, vary significantly as functions of both frequency of excitation and temperature [34], so neglecting these variations can lead to unacceptable levels of error. In this dissertation, the response problem for structures with VEMs is solved at a constant temperature in the frequency domain; only the VEM's dependence on frequency is taken into consideration when solving the frequency response problem (FRP). The target application of this research is a FRP where the frequency dependence of the VEM properties must be taken into account in the formulation of the FRP to obtain an accurate solution.

\subsection{Frequency Response Problem}

The finite element (FE) discretized harmonic response problem for complex structures with frequency dependent damping and stiffness can be written as

$$
\left[-\omega^{2} M+i \omega B(\omega)+K(\omega)\right] X(\omega)=F(\omega)
$$

where $\omega$ is the frequency of the time-harmonic excitation and response, and $i=$ $\sqrt{-1}$. The real-valued mass matrix, $M \in \mathbb{R}^{n_{F} \times n_{F}}$, is symmetric and frequencyindependent. The matrix row dimension $n_{F}$ is typically more than one million in order to obtain acceptable accuracy over the frequency range of interest. The 
viscous damping matrix, $B(\omega) \in \mathbb{R}^{n_{F} \times n_{F}}$, and complex-valued stiffness matrix, $K(\omega) \in \mathbb{C}^{n_{F} \times n_{F}}$, are dependent on frequency. All of the system matrices $M, K(\omega)$, and $B(\omega)$ are very sparse and symmetric. The number of load cases $n_{L}$ in the matrix of load vectors, $F(\omega) \in \mathbb{R}^{n_{F} \times n_{L}}$, is typically between ten and several hundreds. The displacement vectors in the matrix $X(\omega) \in \mathbb{C}^{n_{F} \times n_{L}}$ contain responses, which are to be computed at typically hundreds of frequencies between excitation frequency limits $\omega_{\min }$ and $\omega_{\max }$. The coefficient matrix $A(\omega) \equiv-\omega^{2} M+i \omega B(\omega)+K(\omega)$ is complex-valued and indefinite.

\subsection{Computational Challenges}

Computing solutions to Eq. (1.1) is challenging for two primary reasons. The first reason is that the viscous damping matrix $B(\omega)$ and stiffness matrix $K(\omega)$ are likely to vary with frequency. For the case where damping and stiffness do not depend on frequency, solutions of the FRP for all desired load cases and frequencies are typically computed in several hours to possibly overnight depending on the complexity of the problem, solution method, and computer hardware. Generating $B(\omega)$ and $K(\omega)$ matrices from scratch for every frequency would require an unacceptable amount of time and disk space, especially in the automotive industry where results are required in hours instead of days to keep both design schedules and costs reasonable.

The second reason is that solving Eq. (1.1) for tens to hundreds of load cases at hundreds of frequencies either with a direct or iterative method is prohibitively 
expensive for FRPs with millions of FE DOF. Solving Eq. (1.1) on an approximating subspace, that reduces the dimension of the problem while maintaining accuracy of the solution, is the most common approach to reducing the solution cost. So, the challenge is to inexpensively generate a very efficient approximating subspace on which accurate FRFs can be computed over a large frequency range when the material properties are not constant with respect to frequency.

The computational challenges listed above have served as the motivation for this dissertation. The goal of this research is to develop an efficient and accurate approach to solve Eq. (1.1) in terms of accuracy and elapsed time. The solution approach is introduced in two parts in this chapter. First, in Section 1.3, traditional approaches to representing the frequency dependence of damping and stiffness in the FRP are discussed followed by the introduction of an interpolation approach that eliminates the need to generate damping and stiffness matrices at every frequency. Second, in Section 1.4, existing approximating subspaces are introduced followed by the introduction of the approximating subspace used in this research.

\subsection{Representing the Frequency Dependence of Damp- ing and Stiffness in the FRP}

The frequency dependence of the damping and stiffness matrices in Eq. (1.1) is often either ignored by assuming they are constant for the entire frequency range of interest or approximated crudely by assuming they are constant over portions of the 
frequency spectrum, e.g., 0-200 Hz, 200-400 Hz, etc. Assuming the damping and stiffness matrices are constant or piecewise constant functions of frequency can yield inaccurate solutions. A variation of this approach, called the modal strain energy (MSE) method, assumes the stiffness matrix is constant with respect to frequency and corrects the equations of motion with modal damping terms to account for the frequency dependence in the FRP. The MSE method is the most popular approach in industry today because it is easy to implement and generates solutions quickly, but the results can be fairly inaccurate $[34,33]$. Other approaches commonly referred to as internal variable approaches increase the size of the FRP by adding non-physical "dissipation" coordinates. The more commonly known internal variable approaches are the Golla, Hughes, and McTavish (GHM) and anelastic displacement field (ADF) methods. Internal variable approaches are not attractive for computing solutions for FE models with millions of degrees of freedom (DOF) because these approaches can significantly increase the dimension of the FRP [30].

The approach taken in this dissertation is to interpolate the frequency dependent damping and stiffness matrices between their values at perhaps a half dozen sampling frequencies. The number of sampling frequencies required depends on how much the damping and stiffness matrices vary with frequency. The advantage to this interpolation approach is that complex structures with many different types of frequency dependent materials can be accurately represented with a half dozen damping and stiffness matrices. Overall, using interpolation functions reduces the number of FE matrices generated to a number much smaller than the number of 
frequencies at which solutions are needed.

\subsection{Solving the Frequency Response Problem}

The most common approximating subspace in vibration analysis contains a truncated set of eigenvectors. The eigenvectors are typically computed from the realvalued frequency independent eigenvalue problem

$$
\operatorname{Re}\left\{K\left(\omega_{n}\right)\right\} \Phi=M \Phi \Lambda
$$

where the diagonal matrix $\Lambda$ contains real-valued eigenvalues and $\Phi$ contains realvalued eigenvectors. The real-valued stiffness matrix $\operatorname{Re}\left\{K\left(\omega_{n}\right)\right\}$ is evaluated at a particular or nominal frequency $\omega_{n}$ whose choice can affect the accuracy of the FRP solutions. Approximate solutions to Eq. (1.1) can be inaccurate when computed on an approximating subspace that contains only a truncated set of eigenvectors. One approach to improve the accuracy of these approximate solutions is to enrich the approximating subspace.

Approaches developed by Balmès are the most similar to the approach presented in this research $[5,7,9]$. He generated various approximating subspaces that in some cases resulted in accurate FRFs. In his approaches, he creates approximating subspaces directly from the FE subspace. The cost of generating an approximating subspace on the FE subspace would be prohibitive for FE models with millions of FE DOF. Also, his enrichment techniques increase the dimension of the approximating subspace to at least twice the dimension of the eigenspace. 
Doubling the dimension of the approximating subspace dramatically increases the cost of factoring a modal coefficient matrix. For the solution approach developed in this dissertation, we want to avoid these cost prohibitive computations on the FE subspace and large increases in the dimension of the approximating subspace.

The solution approach taken here is to reduce the cost of factoring and solving the reduced FRP by minimizing the dimension of the approximating subspace without losing solution accuracy. Hundreds to thousands of eigenvectors are replaced with enrichment vectors that represent the quasistatic response to loads and to dashpot forces, and approximate dynamic response to loads. The computational costs of generating these enrichment vectors is significantly reduced by computing them on a reduced subspace associated with the automated multilevel substructuring (AMLS) method. The number of enrichment vectors is also minimized by only computing them when they will improve the final solution. Overall, the use of enrichment vectors allows the approximating subspace to be much smaller than if the same accuracy is obtained by raising the cutoff frequency for global eigenpairs. Computing approximate solutions on a smaller approximating subspace dramatically reduces the cost of solving frequency response problems at many frequencies.

The main motivation behind reducing the dimension of the approximating subspace is that projections of frequency dependent damping and stiffness matrices onto an eigenspace generated from Eq. (1.2) become fully populated at most of the frequencies in the FRP. For the largest FRP in this dissertation, factoring these reduced coefficient matrices at 800 frequencies took over $90 \%$ of the total elapsed 
time because the cost of computing a direct solution at any frequency is proportional to the cube of the dimension of the approximating subspace.

\subsection{Outline of Dissertation}

The properties of VEMs, configurations of VEM applications, and FE modeling approaches of VEM configurations are discussed in Chapter 2. Chapter 3 surveys previously developed methods to represent the frequency dependence in the FRP and solve the frequency dependent FRP. Chapter 4 discusses the interpolation approach and the various vectors contained in the approximating subspace. Chapter 5 explains how the approximating subspace is generated. In Chapter 6, the new solution approach is evaluated on several frequency response problems that have VEMs in terms of solution accuracy and elapsed time. Finally, Chapter 7 discusses conclusions and possible future work. 


\section{Chapter 2}

\section{Properties and Configurations of Viscoelastic Materials}

In this chapter, the discussion starts with how the viscoelastic material (VEM) properties like the Young's modulus, shear modulus, and damping coefficient depend on the frequency of excitation. The next section discusses how VEMs are configured in complex structures like automobiles and aircraft to damp vibrations. The last section explains how the VEMs are typically modeled using the finite element (FE) method.

\subsection{Viscoelastic Material Properties}

VEMs exhibit both elastic (strain energy storage) and viscous (energy dissipation) characteristics. An ideal elastic solid returns to its original shape immediately after 


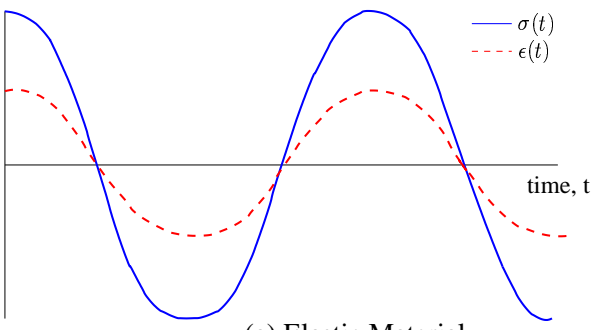

(a) Elastic Material
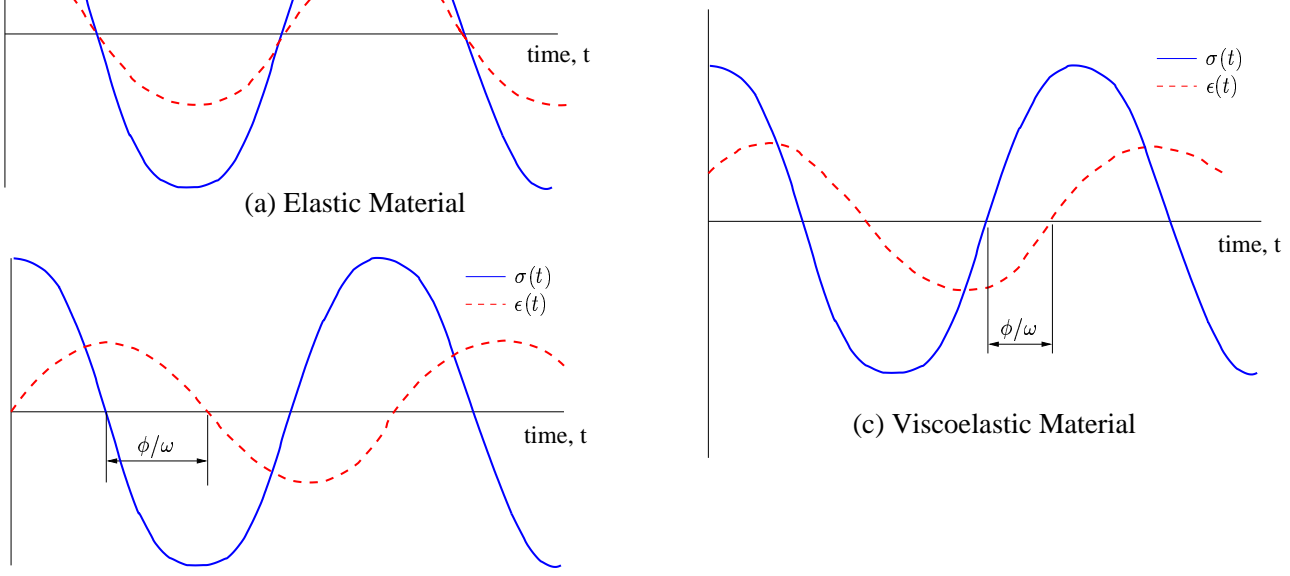

(c) Viscoelastic Material

(b) Viscous Material

Figure 2.1: Cyclic stress-strain relationship vs time for (a) ideal elastic material (b) ideal viscous material (c) VEM [29]

a small load has been removed, so the stress $\sigma(t)$ and the strain $\epsilon(t)$ are in phase with each other (see Fig. 2.1(a)). The stress-strain relation of an ideal viscous material lags by a quarter cycle resulting in strain building up slowly from an applied load (see Fig. 2.1(b)). Since VEMs exhibit both properties to some degree, a time or phase lag $\phi$ between the applied stress and the resulting strain gives VEMs their damping properties (see Fig. 2.1(c)). It is this phase lag that explains why the VEM returns to its original shape slowly enough to at least partially oppose the next cycle of vibration [37]. This opposition removes mechanical energy of vibration from the system by converting the energy to heat. The degree to which most VEMs act viscously or elastically depends primarily on the temperature of the VEM and the frequency of excitation $[34,29]$. 

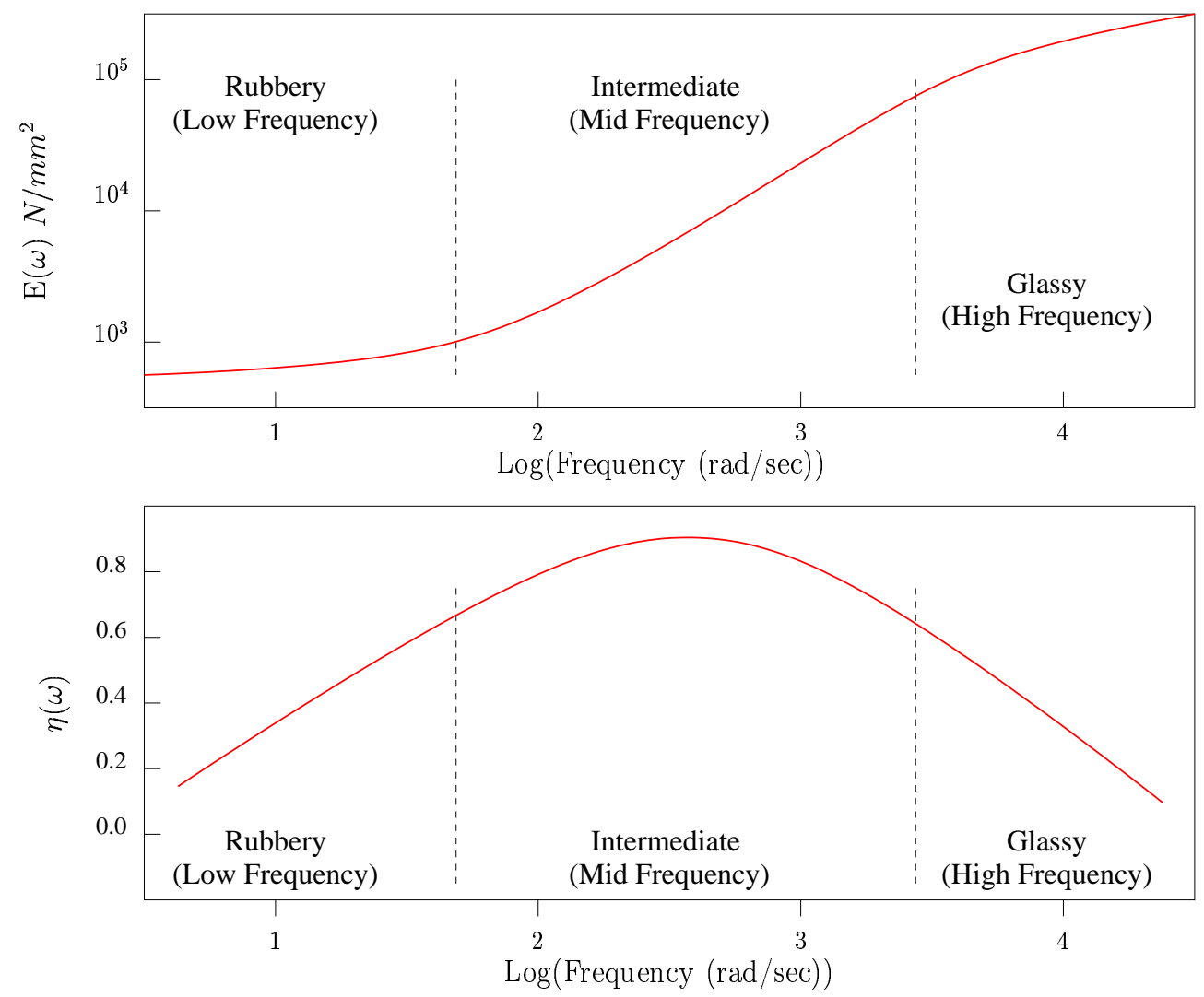

Figure 2.2: Frequency dependence of VEM properties of Young's modulus $E(\omega)$ and loss factor $\eta(\omega)$ for a typical VEM

Figure 2.2 shows the Young's modulus $E(\omega)$ and loss factor $\eta(\omega)$ as functions of frequency for a typical VEM. The loss factor is attributed to the hysterisis phenomenon where energy is removed from the system by internal friction in the material [31]. This non-constant relationship between material properties and frequency makes computing a quick and accurate solution to Eq. (1.1) challenging because the parts of the FE damping and stiffness matrices that represent the VEM are likely to be different at every frequency.

The relationship between normal stress $\sigma$ and normal strain $\epsilon$ for an ideal 
elastic solid is real-valued, linear, and simple:

$$
\sigma=E \epsilon
$$

where $E$ is Young's modulus [34]. For most elastic solids, $E$ does not vary significantly with the frequency of excitation.

For VEMs, the relationship between stress and strain depends on the time history of stress. In the time domain, the stress can be computed as a convolution with the strain history. Using a Fourier transform, one can simply relate stress and strain with an equivalent linear relationship in the frequency domain by

$$
\sigma(\omega, T)=E(\omega, T) \epsilon(\omega)
$$

where $E(\omega, T)$ is complex-valued and depends on the frequency of excitation and temperature. The real part of $E(\omega, T)$ defines the stiffness and the imaginary part of $E(\omega, T)$ defines the damping of the VEM.

The Young's modulus, shear modulus, and damping factor values are measured experimentally by the VEM manufacturer or a testing lab. Numerous functions, such as rational fractions or fractional derivatives, have been developed to fit smooth curves through the experimental data $[9,34,18]$. For this research, the Young's modulus or shear modulus and damping factor values are read directly from tabular data provided by the VEM manufacturer to generate the complex-valued stiffness matrix $K(\omega)$ at the different sampling frequencies that are required for the interpolation approach (see Section 4.1). Next, we will discuss various configurations in which VEMs are incorporated into a structure to damp vibrations. 


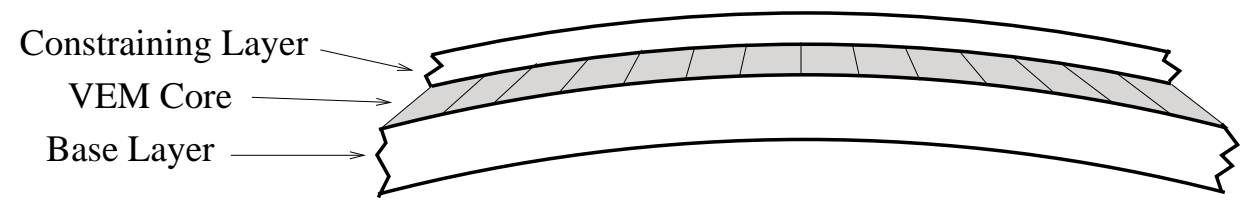

Figure 2.3: CLD product in bending

\subsection{VEM Configuration}

A tuned viscoelastic damper (TVD) configuration is a block of VEM, such as an engine mount, that eliminates unwanted vibrations through shear and extension/compression. VEMs can also be directly sprayed or glued to an existing structural surface, such as a door or roof panel, to damp unwanted vibrations. This damping configuration is called free-layer damping (FLD) and dissipates vibrational energy through extension/compression in the VEM. The FLD configuration tends to be inefficient from a damping to weight point of view, but the material and application costs are generally the lowest of all configurations [34].

For a better damping to weight ratio, an elastic constraining layer or top face can be added to the FLD configuration creating a configuration called the shear or constrained-layer damping (CLD) configuration (see Fig. 2.3 and Fig. 2.4(b)) [34]. CLD products are commercially available (e.g., Quiet Steel ${ }^{\circledR}$, Dynalam, LVDS) and consist of stiff elastic outer skins, such as steel or aluminum, that sandwich a thin viscoelastic core [37]. The CLD products are supplied as rolls and can be stamped, pressed, welded, and punched into a variety of shapes for various components in automobiles or aircraft. Current applications include oil pans, valve covers, door 


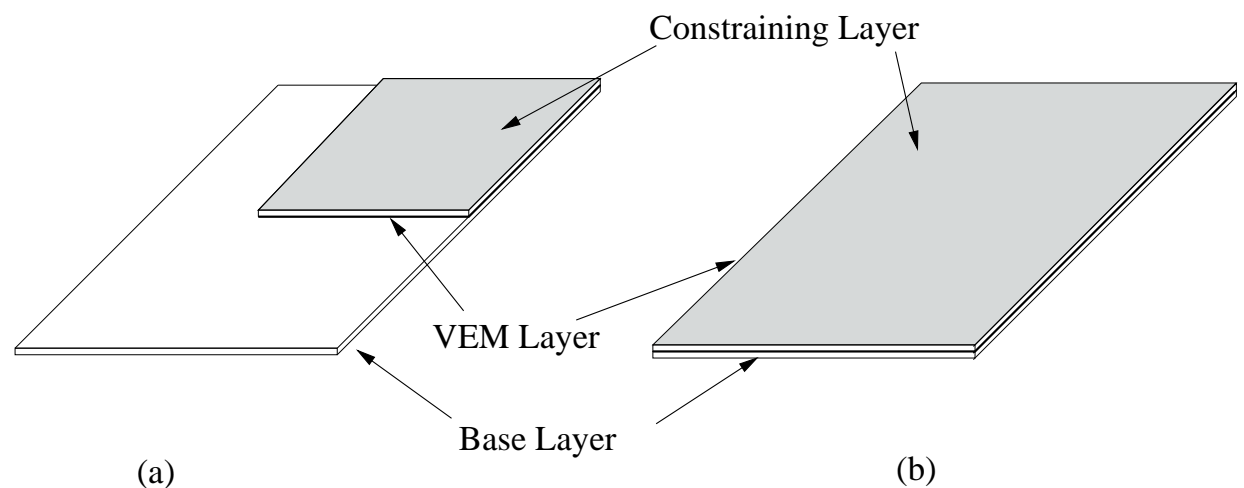

Figure 2.4: (a) Example of a PCL configuration, (b) Example of a CLD configuration

panels, dash panels, windows, stringers, etc. For the best damping results, the core is commonly about $1 / 10^{\text {th }}$ as thick as either constraining layer, and the constraining layers can differ in thickness from each other. This slight difference in thickness between the upper and lower elastic layers increases shear deformation in the VEM when the structure is in bending.

If the elastic top layer and VEM core do not cover the entire base layer, this configuration is referred to as a partially constrained layer (PCL) (see Fig. 2.4(a)). Typically, because the elastic top layer is not fully constrained, the top layer can have larger displacements relative to the bottom layer resulting in larger shear strains in the VEM core. It is these larger shear strains in the VEM core that improve damping in the PCL configuration when compared to the CLD configuration in which both layers are typically constrained on all edges [34].

In both the CLD and PCL configurations, the shear deformation in the VEM core dissipates vibrational energy by converting the energy to heat. At the lower 
frequencies in the rubbery range (see Fig. 2.2), the VEM core is soft (relatively low shear modulus) and undergoes large shear strains resulting in low shear losses which results in a low damping factor. At the higher frequencies the deformation behavior is in the glassy range, the VEM core is stiff and shear strains are resisted more so that relatively smaller shear strains occur, also resulting in a low damping factor. In the mid or intermediate frequency range, there is significant energy dissipated due to shear deformation resulting in a high damping factor [40].

Since the PCL and CLD configurations are very effective and commonly used in industry, the examples and discussions in this dissertation focus on these two configurations. Additionally, any discussions on either CLD or PCL configurations will apply to both configurations because the CLD or PCL configurations are so similar. It is also important to note that the solution approach presented in this dissertation applies to all VEM damping configurations discussed in this chapter. In the next section, the more popular techniques for representing the CLD configuration with finite elements are discussed.

\subsection{Finite Element Modeling Approaches for Constrained Layer Damping Configuration}

Over the last 30 years, researchers have developed numerous FE techniques to model a CLD configuration $[1,34,40,3,33]$. The following is a list of commonly used FE modeling techniques for CLD configurations: 
1. model the elastic faces and VEM core with three-dimensional solid elements,

2. model the upper and lower elastic faces with shell elements and the VEM core with beam elements,

3. model the upper and lower elastic faces with offset shell elements and the VEM core with solid elements, and

4. model the upper and lower elastic faces and VEM core with a shell element.

This list of FE modeling techniques is by no means complete, and the modeling techniques differ primarily on how accurately they represent the dynamic behavior of the VEM core and the number of FE DOF required.

To figure out which FE modeling approach to use, we should look at what is currently being used in the automotive industry. Early in the design cycle of a new automobile, the first FE model typically does not include the effects of damping treatments except for the non-structural mass they add. This is because the global body modes are typically not affected by the damping treatments [3]. Later in the design process, analysts become more concerned with higher frequency effects like local modes, and the effects of the damping treatments can no longer be ignored. At this point, analysts must decide how to modify their FE models to include the VEMs. 


\subsubsection{Equivalent Shell Approach}

The easiest approach for including the dynamic effects of the CLD configuration in an existing FE model is to simply modify the material properties of the existing shell elements to represent the entire CLD sandwich $[3,2,20]$. An equivalent flexural modulus of the CLD configuration is computed to determine how much the material will bend under a given load at several frequencies. The equivalent flexural modulus depends on both the boundary conditions and mode shapes [34] and can be approximated using the best known and simplest to use Ross-Kerwin-Ungar (RKU) equations $[34,3,38]$. The RKU equations apply to beam-like members with pinned boundary conditions, but can be modified to satisfy other section properties and boundary conditions [34]. Better approximations have been developed by Rao, but require correction factors to be applied to each mode of vibration and this approach would make the analysis too difficult for modally dense structures that have thousands of modes [34]. The key advantages of this equivalent shell approach are that the number of FE DOF in the FE model does not increase and the approach can be implemented quickly on an existing model.

\subsection{2 "Shell/Volume/Shell" Approach}

A more accurate, but more difficult to implement, approach is to model the upper and lower elastic faces with offset shell elements and the VEM core with a solid element. This approach is sometimes referred to as the "shell/volume/shell" approach [8]. In an offset shell element, the nodes are displaced away from the neutral surface 


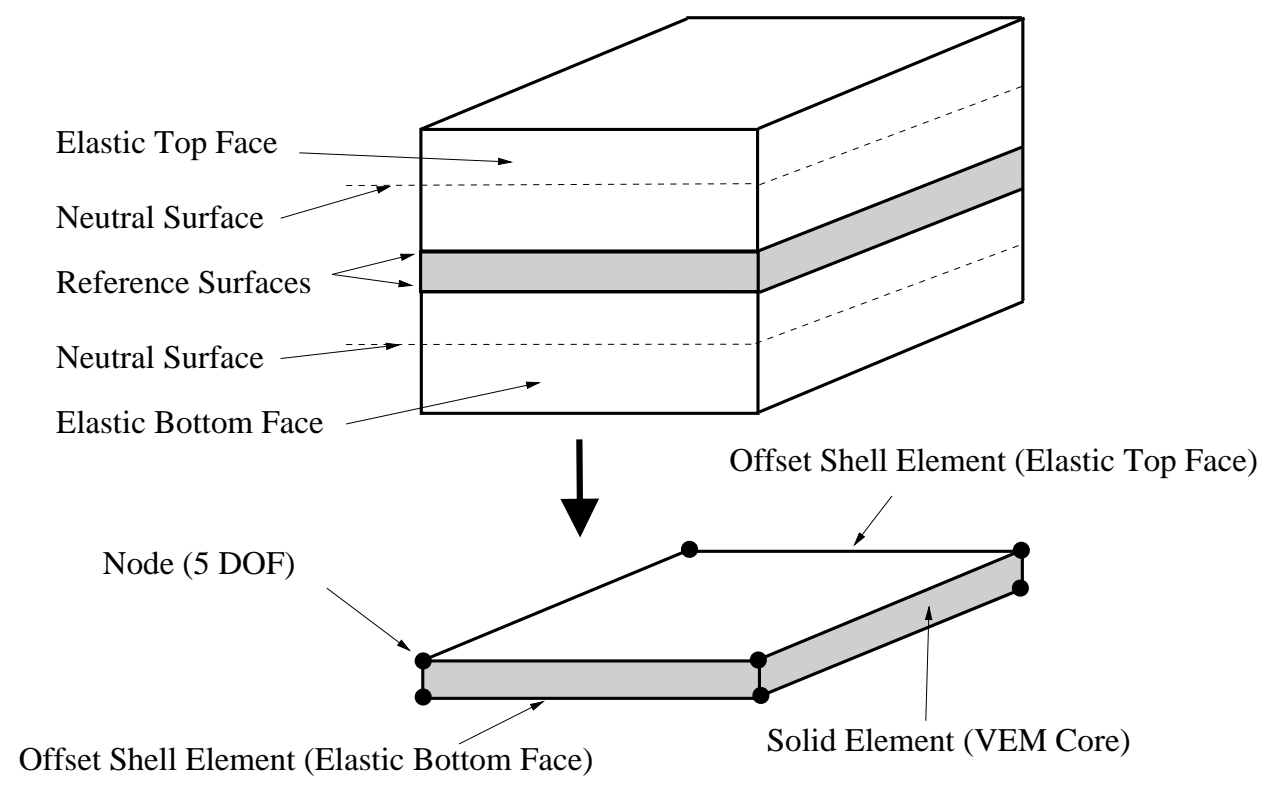

Figure 2.5: FE model of CLD products

by an offset so that they lie on the top and bottom of the VEM core which contains the reference surface (see Fig. 2.5). The offset is defined as the perpendicular distance between the neutral surface of the shell and the reference surface [40].

The advantage of the shell/volume/shell approach is that the nodes on the upper and lower surfaces are allowed to have different horizontal displacements relative to each other. These different horizontal displacements create a shearing effect in the VEM core and are the primary mechanism to damp vibrations in the CLD configuration. The number of nodes in the shell/volume/shell approach increases by a factor of two when compared to the equivalent shell approach. Various commercially available software packages have been developed to exchange existing shell elements with a sandwich of shell/volume/shell elements $[32,6]$. Using these packages can 
significantly decrease the amount of time it takes an analyst to replace single shell elements with a shell/volume/shell sandwich of elements. However, the increase in the number of FE DOF makes the shell/volume/shell approach unattractive if there is a significant amount of CLD material in the structure. For the example FRPs in Ch. 6, both the equivalent shell and shell/volume/shell FE modeling approaches are used. 


\section{Chapter 3}

\section{Survey of Frequency Dependent}

\section{Analysis Methods}

In this chapter, existing methods for representing the frequency dependence of the damping and stiffness in the frequency response problem (FRP) are discussed. The last section of this chapter discusses previously developed methods for solving FRPs with viscoelastic materials (VEMs).

\subsection{Representing Frequency Dependence in the FRP}

Many techniques for representing frequency dependence in a FRP have been developed of which the most common are discussed in this section. For each method, the advantages and disadvantages are discussed in the context of large modally dense FRPs where the frequency dependent properties of VEM must be taken into account 
to obtain accurate solutions.

\subsubsection{Frequency Independent Properties}

It is easiest to assume that the stiffness and damping matrices are constant for the entire frequency spectrum of interest, but the solutions may have unacceptable accuracy. For example, if the Young's modulus for the VEM is selected from the low frequency or rubbery range, as shown in Fig. 2.2, and kept constant, the resulting FRF would be shifted to the left in the higher frequencies compared to a FRF that takes the frequency dependence of the VEM into account. This frequency shift occurs because the resonance frequencies are reduced because the value of the Young's modulus used in the computation of the response is smaller than the actual Young's modulus. For frequency dependent problems, the approach of keeping the frequency dependent properties constant should be limited to frequency ranges narrow enough that there is not a significant loss in accuracy.

\subsubsection{Frequency Ranges of Constant Properties}

Another approach commonly used in industry is to assume that the stiffness and damping matrices are piecewise constant for smaller ranges of the frequency spectrum; like 0-200 Hz, 200-400 Hz, 400-600 Hz, .., etc. If FRFs are computed in each of these frequency regions and pieced together for a complete FRF covering the entire frequency spectrum of interest, there could be discontinuities in the FRFs where the frequency regions are joined together. These discontinuities give an indication 
of the inaccuracy of the approach and a need for smaller frequency ranges.

The advantage to assuming that the damping and stiffness matrices are piecewise constant is that the overall solution is more accurate than a solution obtained assuming the damping and stiffness matrices are constant over the entire frequency range. The disadvantages of assuming piecewise constant matrices, besides the errors is in the solution due to the discontinuities, are that stiffness and damping matrices must be generated for each frequency range and a new eigensolution must be computed for each frequency range if modal analysis is employed.

The computational costs of the eigensolution in each frequency range can be significant especially in the higher frequency ranges, for which many modes must be computed. For example, the computational costs increase because the contribution of eigenvectors whose natural frequencies $\omega_{j}$, where $\omega_{j}$ is the square root of the $j^{\text {th }}$ eigenvalue $\lambda_{j}^{1 / 2}$, are below the frequency range of interest is typically significant and cannot be ignored. For example, to obtain accurate solutions in the frequency range 400 to $600 \mathrm{~Hz}$, the eigenvectors whose natural frequencies are between 0 and 900 $\mathrm{Hz}$ should be computed. Additionally, computational costs climb quickly because the number of modes whose natural frequencies are below a given cutoff frequency is roughly proportional to the square of the cutoff frequency.

\subsubsection{Modal Strain Energy Method}

In 1962, the Modal Strain Energy (MSE) method was introduced by Kerwin and Ungar [24]. The MSE method requires a truncated set of real-valued eigenvectors 
computed from Eq. (1.2). For each eigenvector $\phi_{r}$, the ratio of strain energy in the entire structure and in the VEM are computed. A loss factor $\eta_{r}$ is estimated for each mode from the ratio of the strain energies as shown by

$$
\eta_{r}=\eta\left(\omega_{r}\right) \frac{\phi_{r}^{T} K_{v}\left(\omega_{n}\right) \phi_{r}}{\phi_{r}^{T} K\left(\omega_{n}\right) \phi_{r}} \quad \text { where } \quad r=1, \ldots, n_{E}
$$

where $\omega_{r}=\lambda_{r}^{1 / 2}$ and $n_{E}$ is the number of eigenvectors computed. The loss factor $\eta\left(\omega_{r}\right)$ of the VEM is computed from a function like the fractional derivative or extracted from a table of measured data as discussed in Section 2.1. The value of $\omega_{n}$ can be kept constant when computing the stiffness matrix for the viscoelastic elements $K_{v}\left(\omega_{n}\right)$ and the global stiffness matrix $K\left(\omega_{n}\right)$ which includes the elastic and viscoelastic elements. Otherwise, for more accurate estimations of $\eta_{r}, \omega_{n}$ can be adjusted to a value near $\lambda_{r}^{1 / 2}$ for each eigenvector. The modal loss factors $\eta_{r}$ are computed for $n_{E}$ eigenvectors and applied in an uncoupled modal FRP

$$
-\omega^{2} q_{r}+i \omega \eta_{r} \omega_{r} q_{r}+\omega_{r}^{2} q_{r}=N_{r}
$$

where $q_{r}$ is the $r^{t h}$ modal coordinate, $\omega_{r}$ is the $r^{t h}$ radian natural frequency of the $r^{t h}$ mode, and $N_{r}$ is the modal force for the $r^{t h}$ mode. The advantage to this approach is that the equations of motion are uncoupled by assuming a proportional damping model where the damping in the entire structure is proportional to the mass and/or the stiffness of the structure. Unfortunately, this proportional damping model can be quite unrealistic and lead to inaccurate results.

For more accurate solutions, the eigenvectors of Eq. (1.2) can be computed by setting the nominal frequency $\omega_{n}$ equal to a computed natural frequency $\omega_{r}=\lambda^{1 / 2}$, 
solving again for the eigenpair, and iterating until a convergence criterion is met. This iterative approach has been shown to result in more accurate solutions to the FRP, but the computational costs are significantly higher [41]. The increase in computational costs is due to the cost of extra iterations on the eigenpairs and the fact that the eigenvectors computed from Eq. (1.2) with one value of $\omega_{n}$ are not orthogonal to eigenvectors computed with different values of $\omega_{n}$. The loss of orthogonality makes the FRP in Eq. (3.2) coupled and more expensive to solve. Even though the eigenpairs are arguably more accurate, the accuracy of the frequency response analysis is limited by the proportional damping assumption.

Overall, the MSE method is relatively easy to implement when compared to all of the other methods discussed in this chapter. The MSE method has been implemented in several commercial FE software packages resulting in making the MSE method the primary tool in industry for VEM damping design [28]. The main disadvantages are that the MSE method tends to overpredict the damping and underpredict natural frequencies, leading to significant loss of accuracy when damping is heavy $[33,41]$.

\subsubsection{Revised MSE Method}

In 2002, Xu, Liu, and Wang presented the revised modal strain energy (RMSE) method that improves accuracy in models with high levels of damping. Additionally, the RMSE method does not require iterative analysis to achieve the same level of accuracy as the MSE approach [41]. The RMSE method starts with comput- 
ing a truncated set of eigenvectors from a variation of the real-valued frequency independent eigenvalue problem, Eq. 1.2, written as

$$
\operatorname{Re}\{K(\omega)\} \Phi=M \Phi \Lambda
$$

Each eigenpair is computed with a different stiffness matrix $K(\omega)$ where the value of $\omega$ is determined in a computationally expensive process explained by $\mathrm{Xu}$, Liu, and Wang in their paper [41]. However, the damping factors computed from Eq. (3.1) using the RMSE method are more accurate. When the eigenvalues of the MSE and RMSE methods were compared to eigensolutions for simple beam models, the MSE had relative errors up to 5 percent and RMSE had relative errors up to 3 percent in the first 7 eigenvalues [41].

The MSE methods are popular because they have lower computational costs than most other methods. However, the MSE methods can become computationally expensive if an iterative scheme is used to improve the accuracy of the eigenvectors. The MSE methods do have value in the early stages of product design or optimization when accuracy is not as important. Several other variations of the MSE methods have been developed, but accuracy has always been a major issue. The primary source of error is that the MSE methods typically require proportional damping models to ensure the modal equations of motion are uncoupled. For this research, higher levels of FRF accuracy and little to no user interaction are sought, so the MSE methods are not pursued. 


\subsubsection{Internal Variable Methods}

In the early 90's, internal variable methods were introduced that use additional internal variables to account for frequency dependent damping and stiffness. The Golla, Hughes, and McTavish (GHM) [30, 23, 35] and the Anelastic Displacement Field (ADF) methods are the most common internal variable methods [39]. The internal variable methods do not require modifications to the FE model and can be used for calculating system response to transient loads in both the time and frequency domains. The equations of motion are modified by creating additional degrees of freedom to represent the variation in the VEM properties.

The disadvantage of internal variable methods is that they increase the dimension of the FRP by a factor of at least two [30]. The increase in computational time due to this increase in dimension can be acceptable for smaller FRPs because the solutions using internal variable methods are typically more accurate than the MSE methods. However, these methods are not pursued in this research because the increase in the order of the model would make solving the target problem too computationally intensive to be solved in a reasonable amount of time.

\subsection{Survey of Previously Developed Solution Techniques}

The FRP presented in Chapter 1 is

$$
\left[-\omega^{2} M+i \omega B(\omega)+K(\omega)\right] X(\omega)=F(\omega)
$$


where the coefficient matrix, $A(\omega)=-\omega^{2} M+i \omega B(\omega)+K(\omega)$, is complex-valued, indefinite, and symmetric. Solving the FRP can be computationally demanding even if Eq. (3.4) can be formulated in such a way that the frequency dependent stiffness and damping matrices do not have to be generated at every frequency. Techniques for solving Eq. (3.4) are discussed next.

\subsubsection{Direct Solution}

To compute a direct solution to Eq. (3.4), the coefficient matrix is factored at every frequency. Typically, the sparse coefficient matrices are reordered to reduce fillin, which significantly reduces the amount of required memory to solve the FRP. Computing direct solutions to a FRP with millions of FE DOF can be challenging for most of today's computers.

\subsubsection{Iterative Solution}

Iterative methods are attractive because they have the advantage that they typically do not require factorization of the coefficient matrix. Iterative methods use successive approximations to obtain more accurate solutions at every step until some measure of convergence can be achieved. The main concerns are whether convergence can be achieved and the rate of convergence.

There are two classes of iterative methods: stationary and nonstationary

methods. Jacobi, Gauss-Seidel, successive over relaxation (SOR), and symmetric SOR are the most common examples of stationary methods, but they typically take 
much longer to converge than nonstationary methods. Minimum residual (MINRES) and symmetric LQ (SYMMLQ) are two nonstationary methods that can be applied to complex symmetric indefinite systems of equations [10]. Because the dimension of Eq. (3.4) is typically in the millions, using these iterative methods would be computationally too expensive when solving for tens to hundreds of load cases. However, iterative methods could be considered for computing solutions on reduced subspaces.

\subsubsection{Model Reduction Methods}

Model reduction methods require generating an approximating subspace, projecting the FRP onto the approximating subspace using the Galerkin approach, solving the reduced FRP, and backtransforming the approximate solutions to the FE subspace. The most important requirements for an approximating subspace are that it must be able to accurately represent the response over the entire frequency range of interest, it must be as small as possible, and it must be inexpensive to generate. Numerous model reduction techniques have been developed over the years, but we will only discuss the reduction techniques that are relevant to industrial frequency-dependent FRPs.

\section{Multi-Model Approximation Approach}

In 1996, Balmès introduced the multi-model approximation approach, in which the approximating subspace contains eigenvectors computed from two or more eigen- 
value problems $[5,7]$. The frequency range of interest is broken up into $n$ ranges, for example $0-200 \mathrm{~Hz}, 200-400 \mathrm{~Hz}, \ldots$, etc. Next, the following eigenvalue problem

$$
\operatorname{Re}\left\{K\left(\omega_{j}\right)\right\} \Phi_{j}=M \Phi_{j} \Lambda_{j} \quad \text { for } j=1, . ., n
$$

is solved where $K\left(\omega_{j}\right)$ is kept constant for each frequency range and $\omega_{j}$ is the frequency in the middle of the frequency range. Only the eigenvectors whose natural frequencies are between the lower and upper frequency limits are computed. The approximating subspace is defined by a matrix of eigenvectors $\Phi$ formed by combining the $n \Phi_{j}$ matrices

$$
\Phi=\left[\Phi_{1}, \Phi_{2}, \ldots, \Phi_{n}\right]
$$

Balmès implementation of the multi-model approximation approach did not result in accurate FRFs because $\Phi^{T} \operatorname{Re}\{K(\omega)\} \Phi$ was assumed to be diagonal when it actually was not diagonal. All of the columns of $\Phi$ are not orthogonal to each other with respect to the stiffness matrix because each $\Phi_{j}$ matrix is computed from Eq. (3.5) where the stiffness matrix $K\left(\omega_{j}\right)$ is only held constant for each frequency range $[36,4]$.

\section{Pseudo-Normal Mode Approach}

In 1998, Balmès introduced the pseudo-normal mode approach as an improvement to the multi-model approach [9]. The pseudo-normal mode approach starts with computing a truncated set of eigenvectors from Eq. (1.2) where $\omega_{n}$ remains fixed. 
Vectors, called "pseudo-normal" vectors, are computed by perturbing each eigenvector $\phi_{j}$ to account for the frequency dependence of the problem. The pseudo-normal vectors $\phi_{p_{j}}$ are calculated from

$$
\phi_{p_{j}}=\left[\operatorname{Re}\left\{K\left(\omega_{j}\right)\right\}\right]^{-1} \operatorname{Re}\left\{K_{v}\left(\omega_{j}\right)\right\} \phi_{j} \quad \text { for } j=1, . ., n
$$

where $n$ is the number of eigenvectors computed from Eq. (1.2) and $\operatorname{Re}\left\{K_{v}\left(\omega_{j}\right)\right\}$ is the real-valued stiffness matrix for the elements with VEM. In this approach, an inverse of the stiffness matrix $\operatorname{Re}\left\{K\left(\omega_{j}\right)\right\}$ is computed for every pseudo-normal vector $\phi_{p_{j}}$ where $\omega_{j}$ is close to $\lambda_{j}^{1 / 2}$.

All of the pseudo-normal vectors $\phi_{p_{j}}$ are orthogonalized against the frequency independent eigenvectors $\phi_{j}$ and each other with respect to the mass matrix $M$. The approximating subspace contains both the pseudo-normal vectors $\phi_{p}$ and the frequency independent eigenvectors $\phi_{j}$.

In his 1998 paper, approximate solutions computed using the pseudo-normal mode approach were significantly more accurate than those solutions computed using the multi-model approach. However, the cost of computing the inverses in Eq. (3.7) for all of the pseudo-normal vectors makes the pseudo-normal mode approach prohibitive. Balmès did reduce the overall cost of the pseudo-normal mode approach by reusing the inverse $\left[\operatorname{Re}\left\{K\left(\omega_{j}\right)\right\}\right]^{-1}$ in Eq. (3.7) to compute several pseudo-normal vectors whose natural frequencies were closely spaced. For the example problem in the 1998 paper, the dimension the eigenspace was 49 and using the pseudo-normal mode approach resulted in the approximating subspace being enriched to 109 [36]. 
The pseudo-normal mode approach is not considered for this research for two reasons. First, there is typically at least a two-fold increase in the dimension of the approximating subspace. For the target FRPs in this research that requires thousands of eigenvectors for accurate solutions, a two-fold increase in the dimension of the approximating subspace would make the cost of generating the enrichment to the approximating subspace and solving the FRP on the approximating subspace unacceptable. The second reason the pseudo-normal mode approach is not considered is that the pseudo-normal vectors are computed on the FE subspace. For FRPs with FE dimensions in the millions, computing pseudo-normal vectors from Eq. (3.7) would be computationally too expensive.

Like the approaches created by Balmès, the solution approach presented in this dissertation also enriches the approximating subspace. However, in our approach we minimize the dimension of the eigenspace and the cost of computing the enriching vectors. In the next chapter, we discuss this new approach to solving the FRP with frequency dependent materials. 


\section{Chapter 4}

\section{New Approach to Solving the}

\section{FRP with Frequency Dependent}

\section{Materials}

In the first section of this chapter, the interpolation scheme that was introduced in Chapter 2 is discussed in detail. The second section of this chapter describes the various vectors contained in the approximating subspace. The details of how the approximating subspace is actually generated on the reduced subspaces associated with the automated multilevel substructuring (AMLS) method are left to Chapter 5. 


\subsection{Interpolation Scheme}

The FRP, Eq. (1.1) from Chapter 1, is

$$
\left[-\omega^{2} M+i \omega B(\omega)+K(\omega)\right] X(\omega)=F(\omega)
$$

The interpolation scheme starts with a representation of $K\left(\omega_{n}\right)$ written as

$$
K\left(\omega_{n}\right)=(1+i \gamma) \operatorname{Re}\left\{K\left(\omega_{n}\right)\right\}+i K_{s}\left(\omega_{n}\right)
$$

where $\omega_{n}$ is a chosen nominal frequency. The representative global structural damping coefficient $\gamma$ is typically the damping coefficient of the predominant material in the structure, like steel or aluminum. The real part of the complex-valued symmetric stiffness matrix $\operatorname{Re}\left\{K\left(\omega_{n}\right)\right\}$ represents the stiffness of the structure. The structural damping matrix $K_{s}\left(\omega_{n}\right)$ represents local deviations from $\gamma \operatorname{Re}\left\{K\left(\omega_{n}\right)\right\}$ at the element level.

To approximate $K(\omega)$ as a function of any frequency, the deviation of $K(\omega)$ from $K\left(\omega_{n}\right)$ is defined as

$$
\Delta K(\omega)=K(\omega)-K\left(\omega_{n}\right)
$$

This deviation is sampled at several frequencies $\omega_{j}$ and interpolated using realvalued interpolation functions $g_{j}(\omega)$ (see Fig. 4.1). Each sample $K_{j}$ of the deviation $\Delta K(\omega)$ is expressed as

$$
K_{j}=K\left(\omega_{j}\right)-K\left(\omega_{n}\right)=K\left(\omega_{j}\right)-\left[(1+i \gamma) \operatorname{Re}\left\{K\left(\omega_{n}\right)\right\}+i K_{s}\left(\omega_{n}\right)\right]
$$

Then, $K(\omega)$ is approximated as 


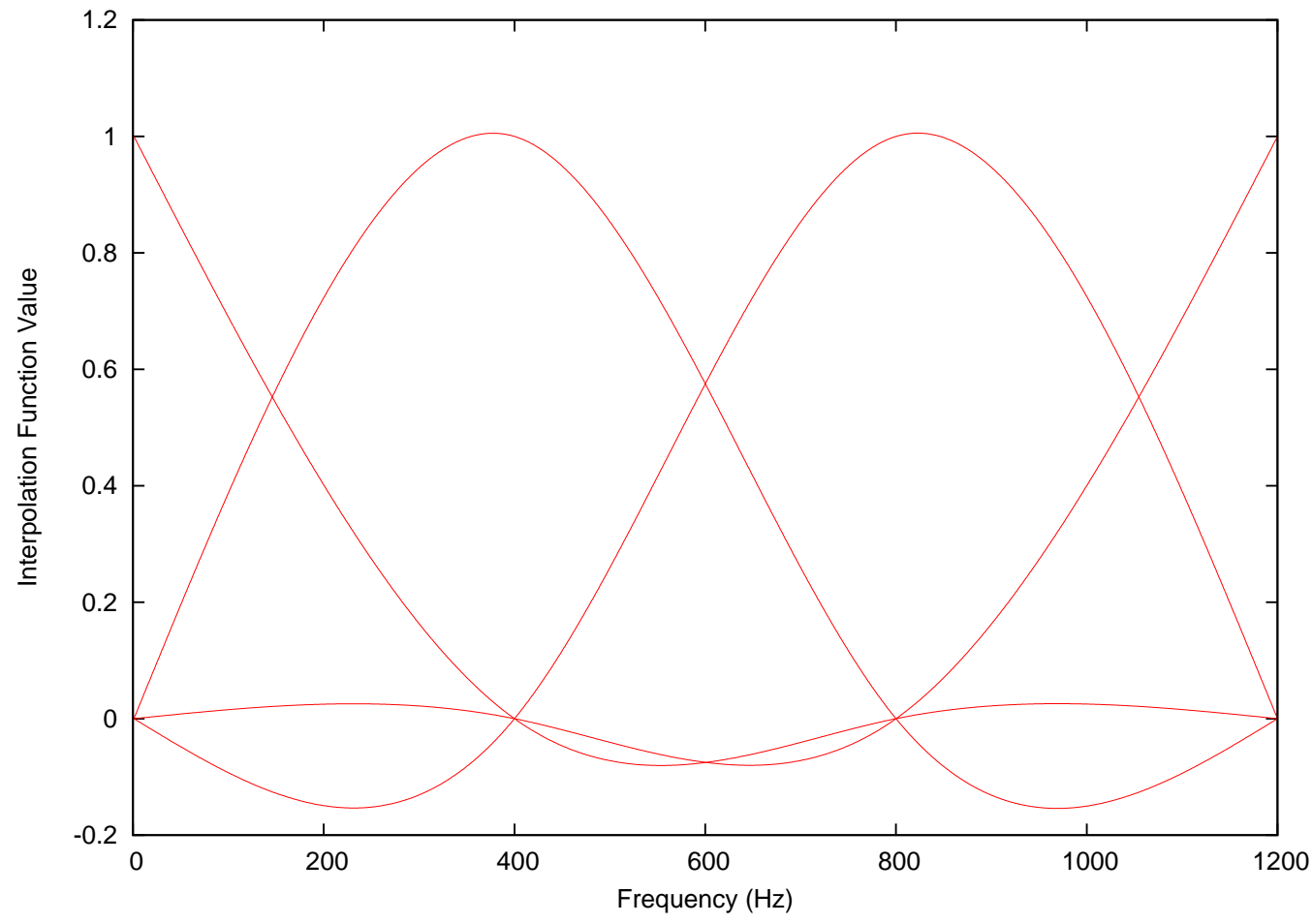

Figure 4.1: Example cubic spline interpolation functions for 4 sampling locations: $0,400,800$, and $1200 \mathrm{~Hz}$

$$
K(\omega) \approx(1+i \gamma) \operatorname{Re}\left\{K\left(\omega_{n}\right)\right\}+i K_{s}\left(\omega_{n}\right)+\sum_{j=1}^{q} g_{j}(\omega) K_{j}
$$

where $q$ is the number of interpolation points. Any smooth interpolation function can be selected that meets the following conditions

$$
\sum_{j=1}^{q} g_{j}(\omega)=1 \quad \text { for any } \omega \text { in the range }
$$

and

$$
g_{j}\left(\omega_{k}\right)=\delta_{j k} \quad \text { for } j, k=1, \ldots, q
$$

where the Kronecker delta is defined as $\delta_{j k}=1$ when $j=k$ and $\delta_{j k}=0$ when $j \neq k$. 


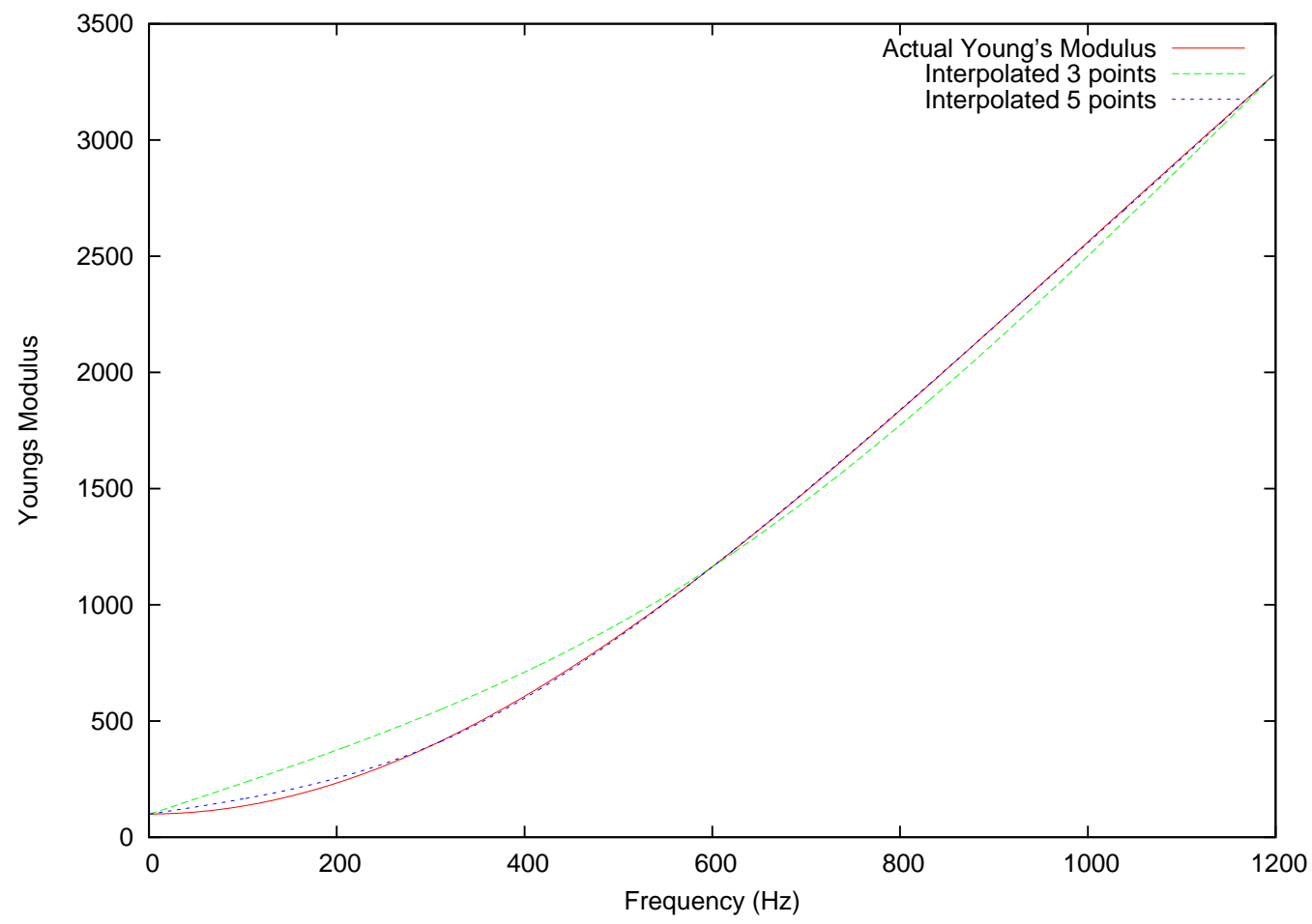

Figure 4.2: Comparison of the interpolated Young's modulus and the actual Young's modulus

Figure 4.1 shows the cubic spline interpolation functions selected for this research.

Figure 4.2 compares the Young's modulus of the interpolated function for three and five equally spaced sampling points against the actual Young's modulus of the VEM. At the three sampling points $(0,600$, and $1200 \mathrm{~Hz})$, the interpolated Young's modulus will have the same value as the actual VEM. This approach of equally spacing the sampling frequencies seems good until one compares the interpolated and actual damping factors (see Fig. 4.3). The most accurate interpolated function is one where the user chooses the sampling frequencies to get the best fit as 


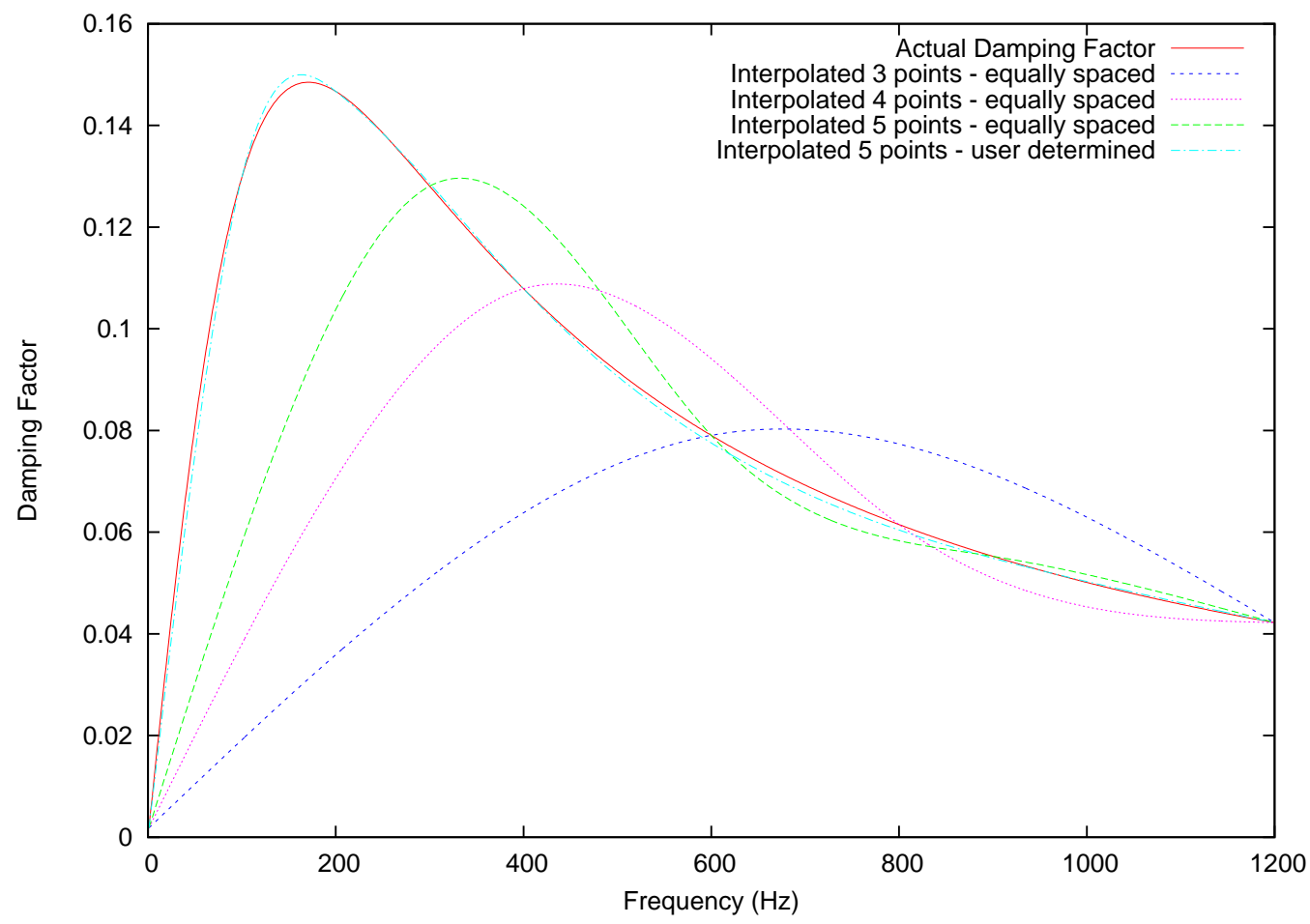

Figure 4.3: Comparison of the interpolated and the actual damping factors

compared to the interpolated functions with equally spaced sampling points. So, if one is careful in selecting the sampling points, the number of required interpolation locations can be reduced.

The approach of interpolating between known values for a stiffness matrix is also applied to the viscous damping matrix, where the frequency dependent viscous damping matrix can be rewritten as

$$
B(\omega) \approx B\left(\omega_{n}\right)+\sum_{j=1}^{p} h_{j}(\omega) B_{j}
$$


The $p$ constant real-valued viscous damping matrices are calculated from

$$
B_{j}=B\left(\omega_{j}\right)-B\left(\omega_{n}\right)
$$

By substituting Eq. (4.5) and Eq. (4.8) into Eq. (4.1), the interpolated FRP can be written as

$$
\begin{gathered}
{\left[-\omega^{2} M+(1+i \gamma) \operatorname{Re}\left\{K\left(\omega_{n}\right)\right\}+i \omega B\left(\omega_{n}\right)+i K_{s}\left(\omega_{n}\right)\right.} \\
\left.+i \omega \sum_{j=1}^{p} h_{j}(\omega) B_{j}+\sum_{j=1}^{q} g_{j}(\omega) K_{j}\right] X(\omega)=F(\omega) .
\end{gathered}
$$

The advantages of this interpolation approach are that the $B_{j}$ and $K_{j}$ matrices only have to be generated one time at their respective sampling frequencies and that numerous VEMs can be represented in the same $K_{j}$ matrices if the sampling frequencies are the same. Complex structures like automobiles typically are made from many different types of materials of which there can be many different types of VEMs. The next step is to generate an approximating subspace on which we can solve the interpolated FRP, Eq. (4.10), accurately and efficiently.

\subsection{Approximating Subspace}

To reduce the cost of solving the FRP on an approximating subspace, we want to minimize the dimension of the approximating subspace without reducing solution accuracy. This dimensional reduction is achieved by replacing hundreds to thousands of eigenvectors with a significantly smaller number of enrichment vectors called the residual flexibility vectors (RFVs), damping deformation vectors (DDVs), and 
dynamic response vectors (DRVs). The computation of the eigenvectors, RFVs, DDVs, and DRVs is discussed next.

\subsubsection{Eigenvectors}

The eigenspace is defined by a truncated set of eigenvectors obtained from Eq. (1.2). Normally, the global cutoff frequency $\omega_{g}$ is set to the industrial standard $1.5 \omega_{\max }$ where $\omega_{\max }$ is the highest excitation frequency. To minimize the number of eigenvectors computed, $\omega_{g}$ is reduced to $1.1 \omega_{\max }$. The smallest value of $\omega_{g}$ that we could accept was determined by incrementally reducing $\omega_{g}$ to the point that accurate solutions could no longer be computed on the approximating subspace, even with enrichment.

Including eigenvectors whose natural frequencies are slightly above $\omega_{\max }$ in the approximating subspace is important to the accuracy of approximate solutions because these eigenvectors typically make a large contribution in the response. It is important to note that even with the reduction in $\omega_{g}$, the number of eigenvectors is significantly larger than the number of enrichment vectors. For the largest FRP presented in this dissertation, the ratio of the number of eigenvectors compared to the number of enrichment vectors is greater than 150:1.

One of the key issues concerning the eigenspace is the selection of the nominal frequency $\omega_{n}$. Approximate solutions to Eq. (4.10) computed on eigenspaces generated with different values of $\omega_{n}$ can vary greatly. The sensitivity of accuracy of the approximate solutions to $\omega_{n}$ will be analyzed and discussed in Chapter 6 . 


\subsubsection{Residual Flexibility Vectors}

Residual flexibility vectors (RFVs) compensate for the lost contribution of truncated eigenvectors whose natural frequencies are above the global cutoff frequency $\omega_{g}$. The RFVs capture localized deformation in the structure caused by the load. RFVs are computed from static responses $X_{s}$ defined by

$$
X_{s}=\left[R e\left\{K\left(\omega_{n}\right)\right\}\right]^{-1} F_{p}(0) \quad \text { at } p=1, \ldots, n_{L}
$$

where $F_{p}(0)$ is the load matrix for the FRP at $0 \mathrm{rad} / \mathrm{sec}$. The subscript "s" is added to the matrix of static responses for RFVs to differentiate $X_{s}$ from the other response matrices in this dissertation. The cost of computing $X_{s}$ is significantly reduced using a procedure explained in the next chapter. The static response vectors will be called RFVs after they have been orthogonalized against the eigenvectors with respect to $\operatorname{Re}\left\{K\left(\omega_{n}\right)\right\}$

\subsubsection{Damping Deformation Vectors}

Damping deformation vectors (DDVs) account for the effects that the viscous damping elements have on the system response. A matrix of force vectors, $F_{b} \in \mathbb{R}^{n_{F} \times n_{B}}$, where $n_{B}$ is the number of $\mathrm{FE}$ viscous damping elements, is created by replacing each of the viscous damping elements with a pair of opposing forces. The matrix of static response vectors is computed from

$$
X_{b}=\left[\operatorname{Re}\left\{K\left(\omega_{n}\right)\right\}\right]^{-1} F_{b}
$$


A subscript " $\mathrm{b}$ " is added to the matrix of static responses $X_{b}$ for DDVs to differentiate it from the other response matrices. The static response vectors will be called DDVs after they have been orthogonalized against the eigenvectors with respect to $\operatorname{Re}\left\{K\left(\omega_{n}\right)\right\}$. The DDVs, like the RFVs, are inexpensive to compute for the same reasons.

\subsubsection{Dynamic Response Vectors}

Reducing $\omega_{g}$ from $1.5 \omega_{\max }$ to $1.1 \omega_{\max }$ typically decreases solution accuracy most noticeably in the frequency range $0.8 \omega_{\max }$ to $\omega_{\max }$. The matrix of dynamic response vectors $(\mathrm{DRVs}) X_{r}$ is a collection of vectors that are corrections to the approximate solutions to Eq. (4.10) at various frequencies in the frequency range $0.8 \omega_{\max }$ to $\omega_{\max }$. DRVs are included in the approximating subspace to maintain the same level of accuracy as approximate solutions computed when $\omega_{g}$ is $1.5 \omega_{\max }$. Since the cost of computing a solution of Eq. (4.10) is not trivial, the DRVs are only approximated, but to a high level of accuracy. The details of how the eigenvectors, RFVs, DDVs, and DRVs are computed are explained in the next chapter. 


\section{Chapter 5}

\section{Computation of the}

\section{Approximating Subspace}

In this chapter, the algorithms for computing the eigenvectors, the residual flexibility vectors (RFVs), the damping deformation vectors (DDVs), and the dynamic response vectors (DRVs) from reduced subspaces made available by the automated multilevel substructuring (AMLS) method are explained in detail. First, a partially constrained layer (PCL) plate problem is introduced to provide a simple example as

the basis for discussions in this chapter. Then, a short synopsis of the history of the AMLS method is followed by an overview of the AMLS method and software. The remaining sections of this chapter discuss the implementation of the new approach for solving frequency response problems (FRPs) with viscoelastic materials (VEMs). 


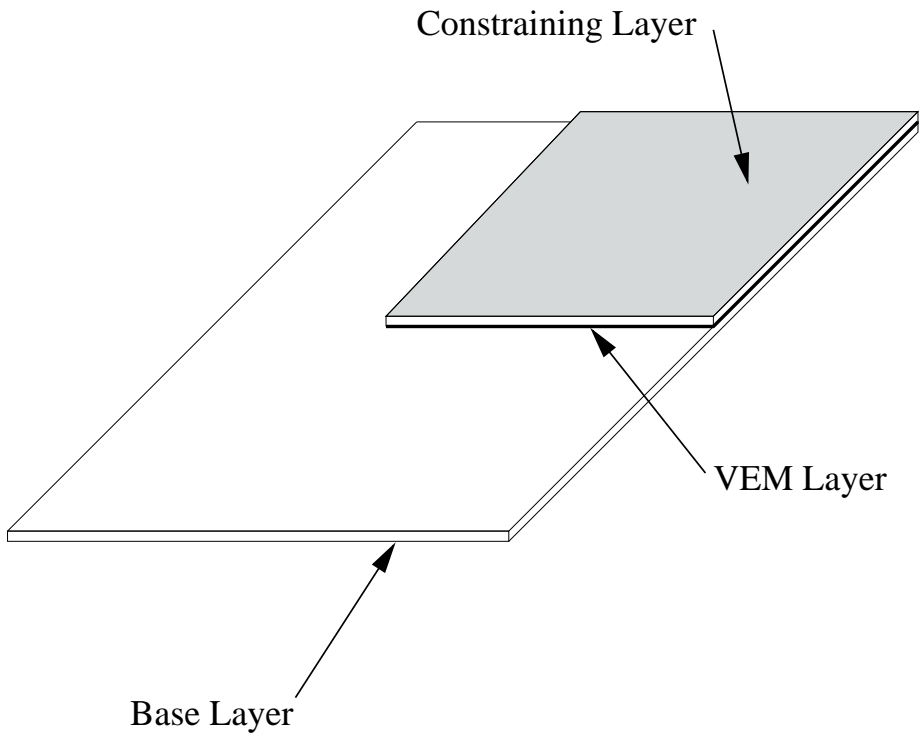

(a)

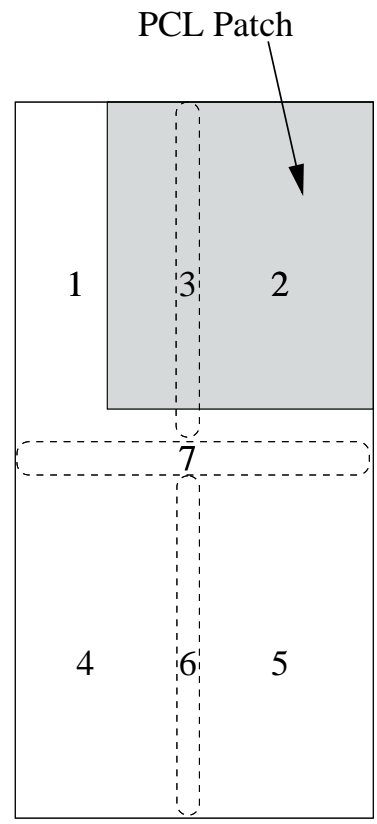

(b)

Figure 5.1: (a) Partially constrained layer (PCL) plate example, (b) Example plate partitioned into seven substructures

\subsection{Partially Constrained Layer Plate Example}

A PCL plate example, shown in Fig. 5.1, is used to clarify the discussions in this chapter. This simple plate example was selected to make the differences between the various reduced damping and stiffness matrices more obvious. This example's VEM core has material properties whose frequency dependence is similar to the material properties shown in Fig. 2.2. 
The interpolated FRP, Eq. (4.10), from Chapter 4 is

$$
\begin{gathered}
{\left[-\omega^{2} M+(1+i \gamma) \operatorname{Re}\left\{K\left(\omega_{n}\right)\right\}+i \omega B\left(\omega_{n}\right)+i K_{s}\left(\omega_{n}\right)\right.} \\
\left.+i \omega \sum_{j=1}^{p} h_{j}(\omega) B_{j}+\sum_{j=1}^{q} g_{j}(\omega) K_{j}\right] X(\omega)=F(\omega) .
\end{gathered}
$$

To simplify the discussion and reduce the number of equations in the first few sections of this chapter, only the transformation of the $M, \operatorname{Re}\left\{K\left(\omega_{n}\right)\right\}$, and $K_{j}$ matrices is discussed because the transformation of the $B\left(\omega_{n}\right), B_{j}$, and $K_{s}\left(\omega_{n}\right)$ matrices is similar to the transformation of the $K_{j}$ matrices. Also, the $\operatorname{Re}\left\{K\left(\omega_{n}\right)\right\}$ matrix will simply be written as $K$ until Section 5.8.1. Additionally, subscript capital letters denote the subspace a matrix or vector has been projected onto. For example, $M_{D}$ is the FE mass matrix projected onto the "distilled" subspace $(\mathcal{D})$. If a capital letter subscript is not present, the matrix or vector is expressed on the FE $\operatorname{subspace}(\mathcal{F})$.

\subsection{History of the Automated Multi-Level Substructur- ing (AMLS) Method}

In 1992, the adaptive multi-level substructuring method was proposed by Bennighof and C. K. Kim [17]. In this 1992 version of AMLS, the partitioning into substructures was done manually, rather than automatically, and the emphasis was on adaptively selecting the substructure modes to include in the model to get a desired level of frequency response accuracy. By 1995, the process of automating the division into substructures had been started, and the name of the method was changed to 
the automated multilevel substructuring (rather than adaptive, but still AMLS) method. Bennighof and Kaplan $[12,13]$ extended the capabilities of the software implementation of the method, and by 1998, automated substructure partitioning had been implemented, and Bennighof, Kaplan, Muller, M. Kim, and C. W. Kim were computing FRFs from a transformed model.

The AMLS method and software implementation continued to mature and became commercially available summer 2001 . Bennighof $[17,11,12,13,16]$, Kaplan [25], C. W. Kim [26], Muller [15, 14], and M. Kim [27] have all made significant improvements to the AMLS method and software over the years, and this has resulted in making the AMLS method a widely used eigensolver in the automotive industry.

The AMLS method extends the Craig-Bampton component mode synthesis approach to multiple levels, with automated division of the structure model into substructures $[22,21,27]$. The reduced subspaces associated with the AMLS method used in this research are called the AMLS subspace or substructure mode subspace $(\mathcal{A})$ and the $\mathcal{D}$ subspace. The AMLS method efficiently reduces the dimension of the FE matrices by projecting them onto the $\mathcal{A}$ and $\mathcal{D}$ subspaces, and it is on these subspaces that the approximating subspace is generated for this research.

\subsection{Overview of the AMLS method}

In this section, we will discuss the dimensional reduction of a typical industrial FRP

that has 10 million FE DOF. It is important to note that the dimensions of the 
AMLS subspaces discussed in this paragraph are just representative round numbers rather than specified subspace sizes. The reduction from the $\mathcal{F}$ subspace to the $\mathcal{A}$ subspace reduces the dimension of the industrial model from 10 million to around 100 thousand. The reduction from the $\mathcal{A}$ subspace to the $\mathcal{D}$ subspace reduces the dimension of the industrial model from 100 thousand to around 40 thousand. About 10 thousand eigenpairs, whose eigenvalues are below $\omega_{g}^{2}$ where $\omega_{g}$ is the global cutoff frequency, can then be approximated from $K_{D} \Phi=M_{D} \Phi \Lambda$ [27].

\subsection{Overview of the Implementation}

An AMLS analysis begins with an FE code generating the mass, stiffness, damping, and load matrices. This process is considered "Phase 1" of the analysis. Then, in Phase 2, the AMLS software reads the matrix data generated in Phase 1 and determines how to divide the FE model into substructures on multiple levels based on the sparsity structure of the matrices. In Phase 3, substructure eigenvalue problems are solved to reduce the FE model to the $\mathcal{A}$ subspace. In Phase 4 , the $\mathcal{D}$ subspace is defined, the solution of the eigenvalue problem $K_{D} \Phi=M_{D} \Phi \Lambda$ is approximated, and the RFVs and DDVs are computed on the $\mathcal{A}$ subspace. Phase 5 expresses the eigenvectors found in Phase 4 in terms of the FE degrees of freedom and transforms damping and load matrices to the modal subspace for solving the modal FRP. If the modal FRP is solved by the AMLS software, it is solved in Phase 6 .

These phases must be extended or replaced to handle frequency dependent 


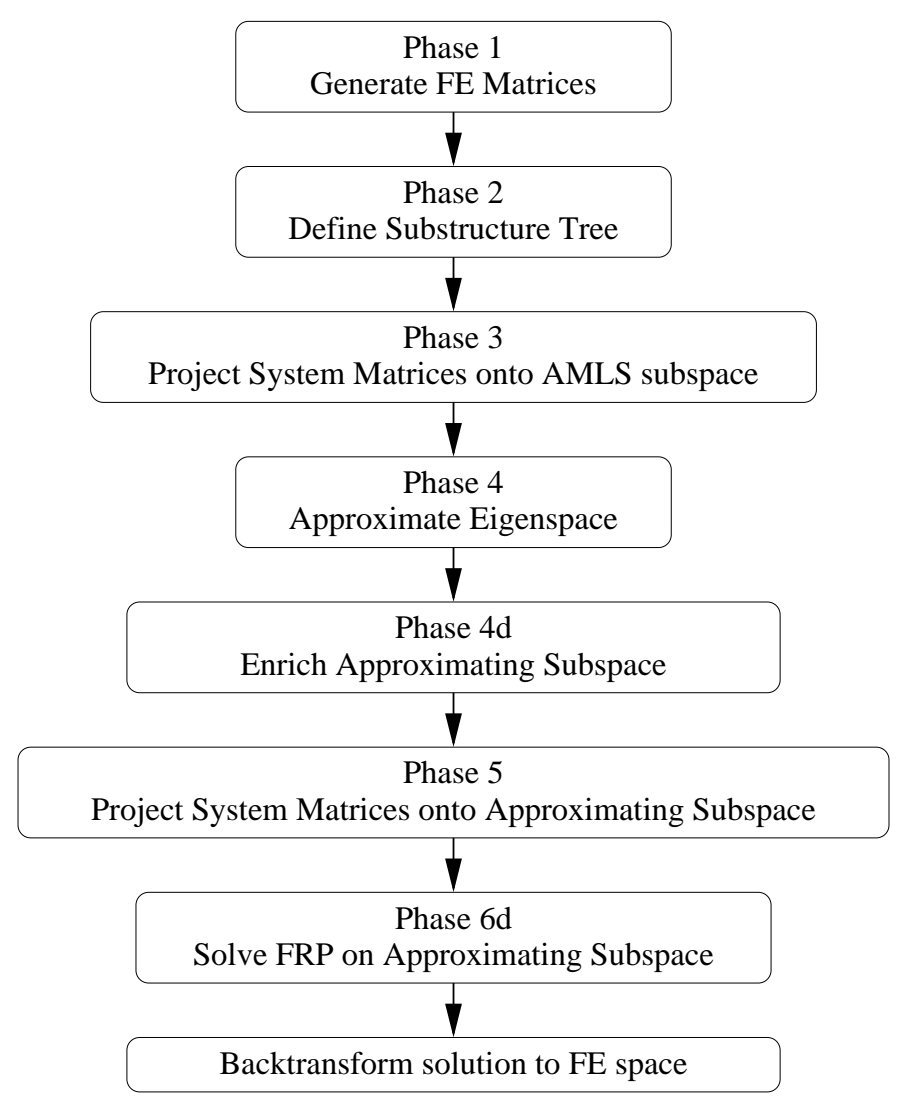

Figure 5.2: Flowchart of the solution sequence

FRPs. In Phase 1 , the $K_{j}$ matrices are generated in the FE subspace. In Phase 3, the $K_{j}$ matrices need to be transformed to the $\mathcal{A}$ subspace along with $M$ and $K$. Phase 4 is modified to obtain DDVs along with the RFVs and the eigenvectors to form the initial approximating subspace $\left(\mathcal{W}_{0}\right)$. Phase $4 \mathrm{~d}$ is added to obtain DRVs in the $\mathcal{D}$ subspace so that they can be included in the approximating subspace $(\mathcal{W})$. Computing DRVs requires reducing the $K_{j}$ matrices to $\mathcal{D}$ and $\mathcal{W}_{0}$ subspaces and reducing the $M$ and $K$ matrices to the $\mathcal{W}_{0}$ subspace. In Phase 5 , the $K_{j}$ matrices are reduced to the $\mathcal{W}$ subspace so that they can be used in Phase $6 \mathrm{~d}$. Finally, Phase $6 \mathrm{~d}$ 


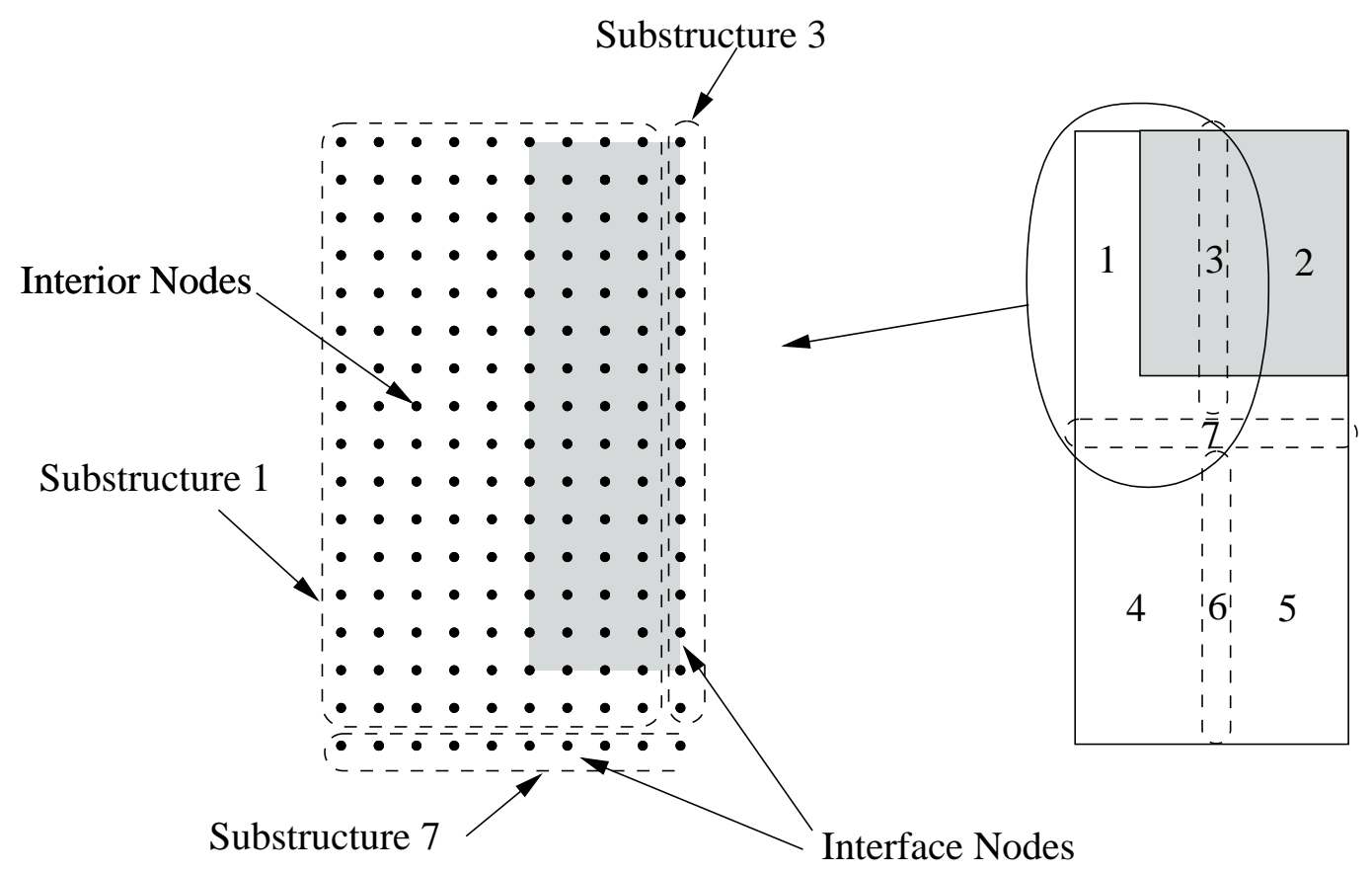

Figure 5.3: Depiction of interior and shared nodes

solves the frequency dependent FRP on the approximating subspace rather than the modal subspace and passes the FRP approximate solutions back to the FE software so that the approximate solutions can be returned to the user in terms of FE DOFs. The results of this approach are discussed in the next chapter, and the details of each phase are explained next, starting with Phase 2.

\subsection{Phase 2: Define Substructure Tree}

Phase 2 uses an open source program called METIS to compute a reordering scheme that applies to both the $M$ and $K$ matrices. METIS partitions the FE mesh to pro- 


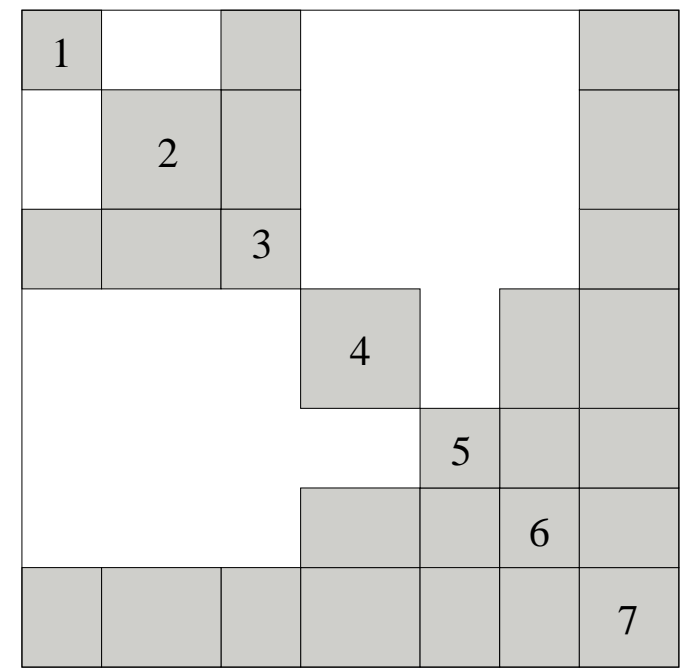

(a)

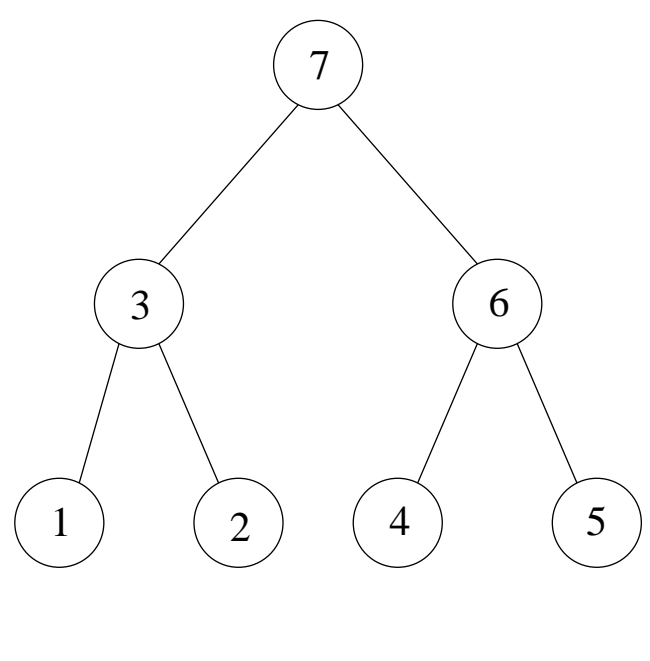

(b)

Figure 5.4: (a) Sparsity pattern $M$ and $K$ after reorder in Phase 2, (b) Substructure tree diagram of $M$ and $K$

duce a fill-reducing ordering based on a multilevel recursive bisection for sparse matrices. For example, Fig. 5.3 shows the model plate divided into seven substructures after recursive bisectioning, and Fig. 5.4(a) shows the sparsity pattern of the reordered $M$ and $K$ matrices. Each shaded block in Fig. 5.4(a) represents the nonzeros in the matrices, and the blocks are typically not the same size.

Next, Phase 2 infers a substructure tree from the reordered FE matrices. Figure 5.4(b) is the substructure tree diagram of $M$ and $K$ that conveniently illustrates the relationships between the substructures. The root substructure is number 7, and all other substructures are descendants of the root.

Substructure 1 is defined to consist of nodes in the interior of the upper left quadrant of the plate, as shown in Fig. 5.3. Substructure 3 consists of the nodes 
at the interface between Substructure 1 and Substructure 2, which corresponds to the upper right quadrant. The nodes or degrees of freedom for Substructure 3 are referred to as "interface" or "shared" nodes or degrees of freedom. Substructures 4, 5, and 6 are similarly defined in the lower half of the plate, and Substructure 7 consists of the nodes at the interface between the upper and lower halves of the plate. This reordering of the system matrices results in the stiffness matrix $K$ having the form

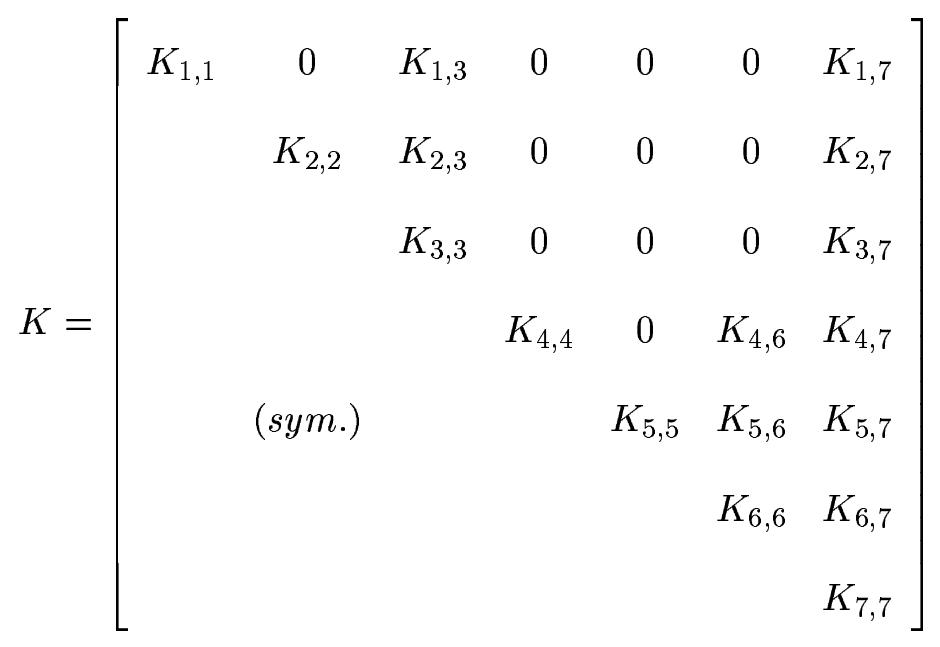

and the mass matrix $M$ having the form

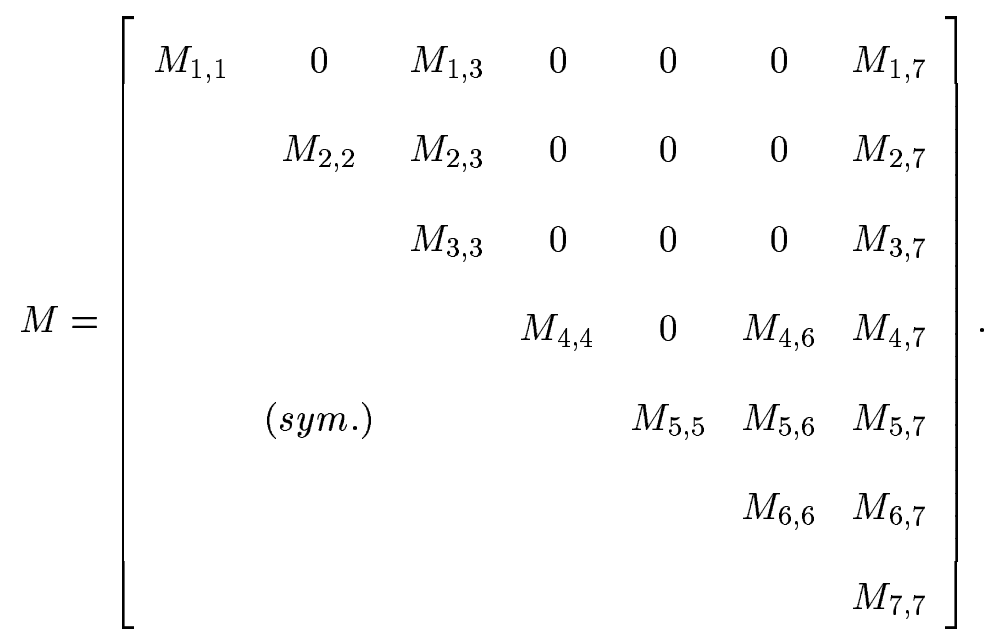


The $K_{j}$ matrix is also reordered and has the form

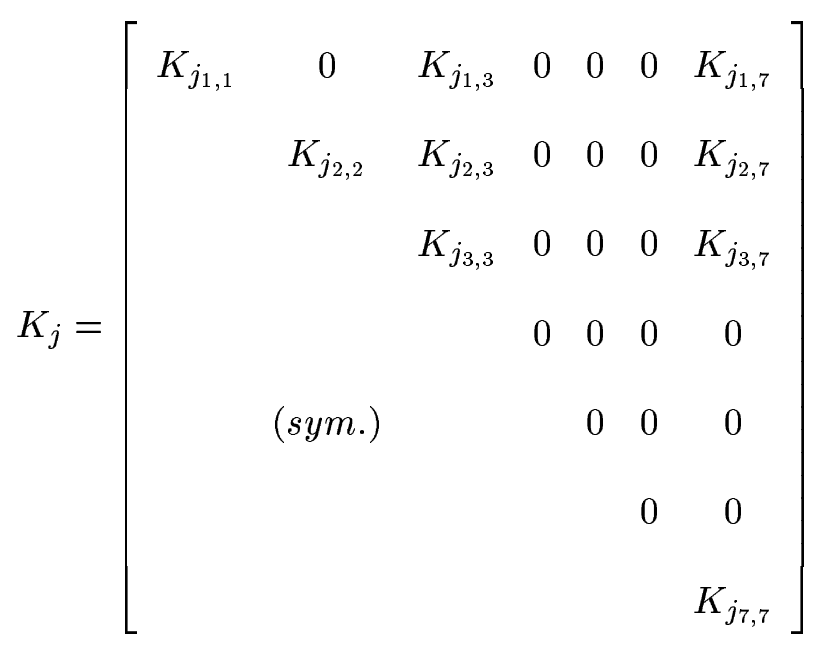

where the submatrices corresponding to substructures 4,5 , and 6 are zero for this example plate problem (see Fig. 5.5a). The purpose of selecting a PCL example that only has VEM on the upper half of the plate is to demonstrate how the partially filled $K_{j}$ matrices are transformed (see Fig. 5.3).

\subsection{Phase 3: Project System Matrices onto AMLS Sub- space}

As mentioned earlier, the purpose of Phase 3 is to solve the substructure eigenvalue problems and reduce the $M, K$, and $K_{j}$ matrices to the $\mathcal{A}$ subspace. First, we will discuss how the $\mathcal{A}$ subspace transformation matrix $T_{A}$ is generated from $T_{A}=$ $T^{(1)} T^{(2)} T^{(3)} \ldots T^{(7)}$ where $T^{(1)}$ is the first substructure transformation matrix, and similarly for substructure transformation matrices 2 through 7 . The final goal of this section is to discuss how the $K, M$, and $K_{j}$ matrices are transformed to the 


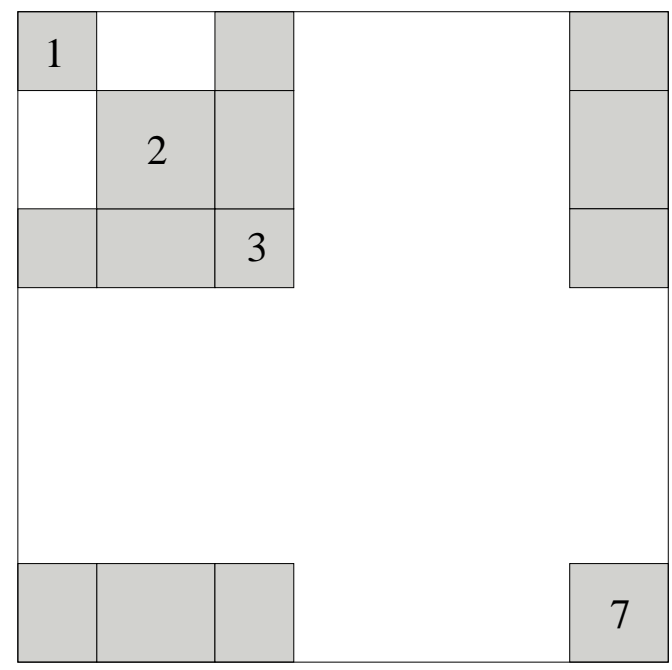

(a)

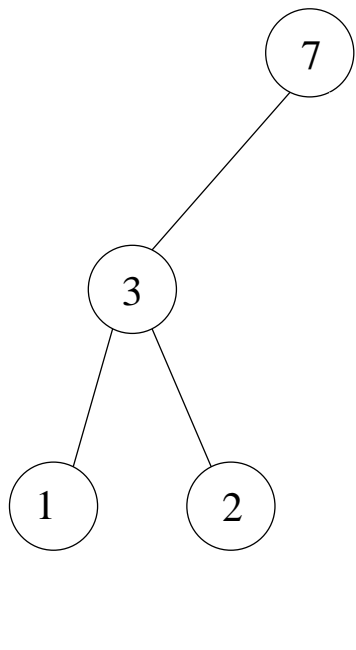

(b)

Figure 5.5: (a) Sparsity pattern of $K_{j}$ after reorder in Phase 2 (b) Substructure tree diagram of $K_{j}$

$\mathcal{A}$ subspace by the transformations $K_{A}=T_{A}^{T} K T_{A}, M_{A}=T_{A}^{T} M T_{A}$, and $K_{j A}=$ $T_{A}^{T} K_{j} T_{A}$, respectively.

The transformation matrix for the first substructure $T^{(1)}$ is formed for the example problem as

$$
T^{(1)}=\left[\begin{array}{ccccccc}
\Phi_{1,1} & 0 & \Psi_{1,3} & 0 & 0 & 0 & \Psi_{1,7} \\
0 & I_{2,2} & 0 & 0 & 0 & 0 & 0 \\
0 & 0 & I_{3,3} & 0 & 0 & 0 & 0 \\
0 & 0 & 0 & I_{4,4} & 0 & 0 & 0 \\
0 & 0 & 0 & 0 & I_{5,5} & 0 & 0 \\
0 & 0 & 0 & 0 & 0 & I_{6,6} & 0 \\
0 & 0 & 0 & 0 & 0 & 0 & I_{7,7}
\end{array}\right] .
$$


This transformation matrix projects substructure 1 onto a reduced subspace defined by the matrix of truncated substructure modes $\Phi_{1,1}$ and the matrix of constraint modes $\Psi_{1,3}$ and $\Psi_{1,7}$. The matrix of substructure eigenvectors $\Phi_{1,1}$ for substructure 1 is computed from the eigenvalue problem

$$
K_{1,1} \Phi_{1,1}=M_{1,1} \Phi_{1,1} \Lambda_{1,1}
$$

where the eigenvectors are mass normalized such that $\Phi_{1,1}^{T} M_{1,1} \Phi_{1,1}=I$. Only the eigenvectors whose eigenvalues are smaller than $\omega_{A}^{2}$ are computed, where $\omega_{A}$ is the substructure cutoff frequency. Since substructure 1 consists of nodes only in the interior of the upper left quadrant of the plate, the eigenvectors describe the motion of the interior DOF relative to the fixed boundaries [22].

Constraint modes are defined as quasi-static responses of the substructure with a unit displacement in one interface DOF and zero displacement in all other interface DOF [22]. The constraint modes for substructure 1 associated with the substructure 3 interface, $\left[\begin{array}{llllll}\Psi_{1,3}^{T} & 0 & I_{3,3} & 0 & 0 & 0\end{array}\right]^{T}$, represent the quasi-static dependence of substructure 1's local DOF on substructure 3's interface DOF, and similarly for the constraint modes of substructure 1 associated with substructure 7 interface, $\left[\Psi_{1,7}^{T} 00000000 I_{7,7}\right]^{T}$. The constraint modes are computed from

$$
\Psi_{1, j}=-K_{1,1}^{-1} K_{1, j}, \quad \text { for } j=3,7 \text {. }
$$


After the first transformation, the resulting stiffness matrix is

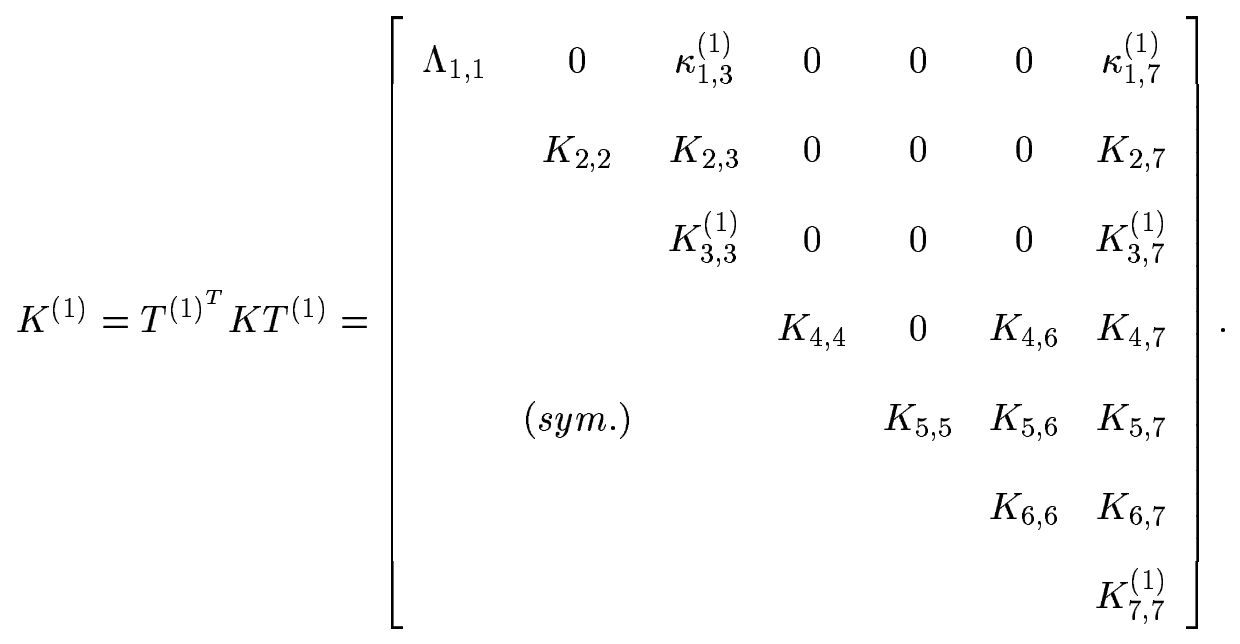

The intermediate products, $\kappa_{1,3}^{(1)}$ and $\kappa_{1,7}^{(1)}$, are defined as

$$
\kappa_{1, j}^{(1)}=\Phi_{1,1}^{T}\left(K_{1,1} \Psi_{1, j}+K_{1, j}\right) \quad \text { for } j=3,7,
$$

but these are equal to zero because of the definition of $\Psi_{1, j}$ in Eq. (5.7). The only other submatrices in $K^{(1)}$ that are different from the corresponding submatrices in Eq. (5.2) are in rows and columns 3 and 7, and similar cancellations come into play in their definitions:

$$
K_{i, j}^{(1)}=K_{i, j}+K_{1, i}^{T} \Psi_{1, j}, \quad \text { for } i, j=3,7 .
$$

For the $M$ and $K_{j}$ matrices, these cancellations do not apply. The transformed submatrices of the mass matrix are given by

$$
\begin{aligned}
& M_{1, j}^{(1)}=\Phi_{1,1}^{T}\left(M_{1,1} \Psi_{1, j}+M_{i, j}\right), \quad \text { for } j=3,7, \quad \text { and } \\
& M_{i, j}^{(1)}=\Psi_{1, i}^{T}\left(M_{1,1} \Psi_{1, j}+M_{1, j}\right)+M_{1, i}^{T} \Psi_{1, j}+M_{1, j}, \quad \text { for } i, j=3,7,
\end{aligned}
$$


and the transformed mass matrix after the first substructure's transformation is

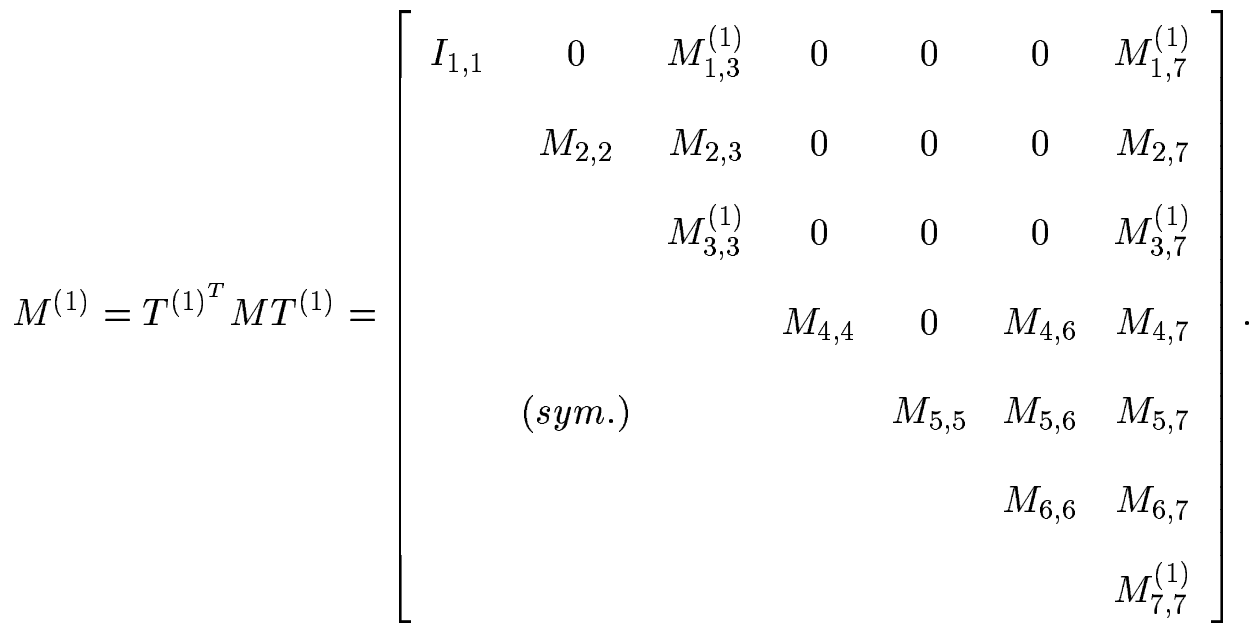

The components of the $K_{j}^{(1)}$ matrix after one transformation are computed from

$$
\begin{aligned}
\left(K_{j}\right)_{1,1}^{(1)} & =\Phi_{1,1}^{T} K_{j_{1,1}} \Phi_{1,1} \\
\sigma_{1, j}^{(1)} & =\Phi_{1,1}^{T}\left(K_{j_{1,1}} \Psi_{1, j}+K_{j_{i, j}}\right) \quad \text { for } j=3,7, \text { and } \\
K_{j_{i, p}}^{(1)} & =\Psi_{1, i}^{T}\left(K_{j_{1,1}} \Psi_{1, p}+K_{j_{1, p}}\right)+K_{j_{1, i}}^{T} \Psi_{1, p}+K_{j_{1, p}} \quad \text { for } i, p=3,7 .
\end{aligned}
$$

The transformed $K_{j}$ matrix is

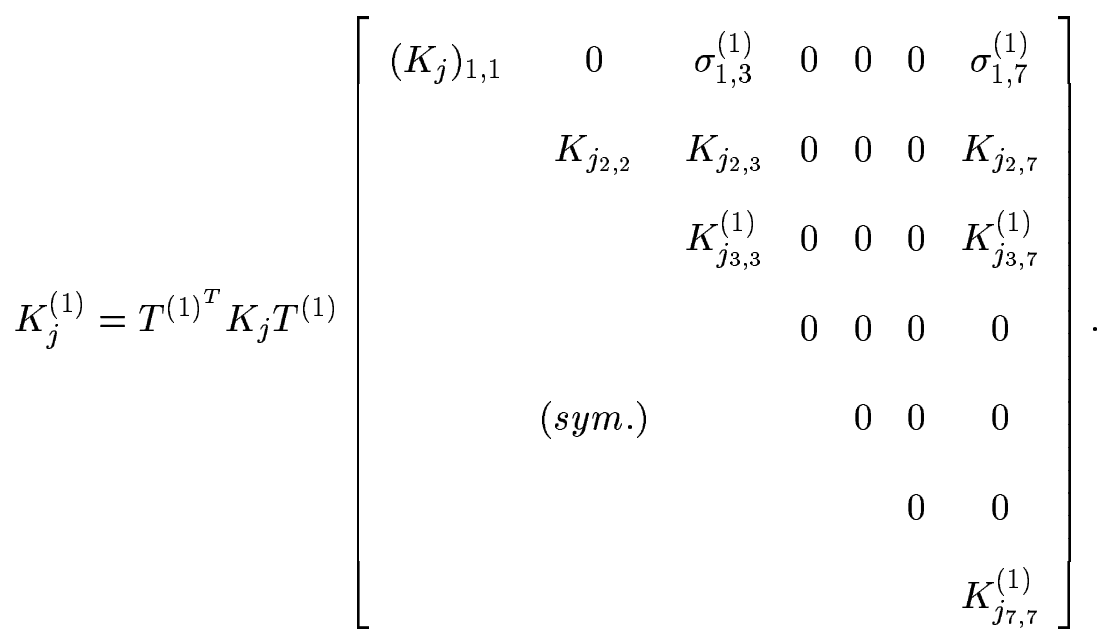


Since the transformation of substructure 2 is very similar to the transformation of substructure 1 , we will not discuss it in detail. The intermediate step of the transformation of substructure 3 shows details that are important to the overall transformation. The transformation matrix $T^{(3)}$ is given by

$$
T^{(3)}=\left[\begin{array}{ccccccc}
I_{1,1} & 0 & 0 & 0 & 0 & 0 & 0 \\
0 & I_{2,2} & 0 & 0 & 0 & 0 & 0 \\
0 & 0 & \Phi_{3,3} & 0 & 0 & 0 & \Psi_{3,7} \\
0 & 0 & 0 & I_{4,4} & 0 & 0 & 0 \\
0 & 0 & 0 & 0 & I_{5,5} & 0 & 0 \\
0 & 0 & 0 & 0 & 0 & I_{6,6} & 0 \\
0 & 0 & 0 & 0 & 0 & 0 & I_{7,7}
\end{array}\right]
$$

where $\Psi_{3,7}$ and $\Phi_{3,3}$ satisfy

$$
\begin{aligned}
\Psi_{3,7} & =-K_{3,3}^{(2)^{-1}} K_{3,7}^{(2)} \quad \text { and } \\
K_{3,3}^{(2)} \Phi_{3,3} & =M_{3,3}^{(2)} \Phi_{3,3} \Lambda_{3,3} .
\end{aligned}
$$

Applying transformation matrix $T^{(3)}$ to $K^{(2)}$ and $M^{(2)}$ and taking into account the 
cancellation that occurs, the transformed $K^{(3)}$ is

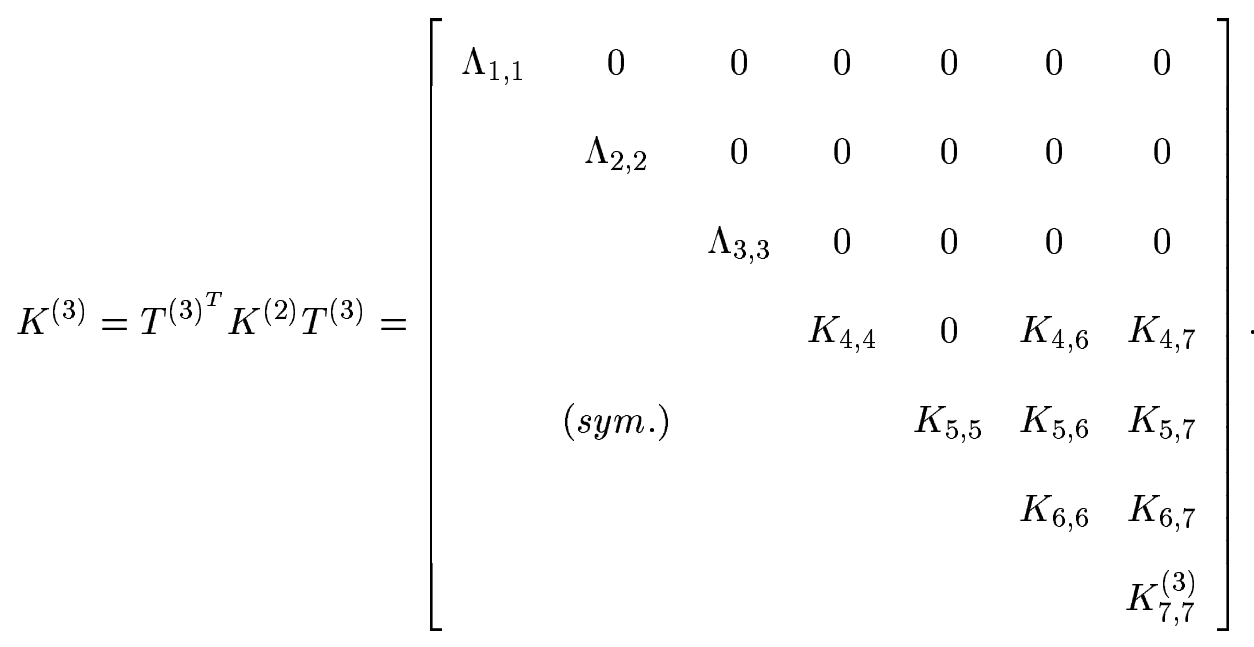

The transformed mass matrix $M^{(3)}$ is

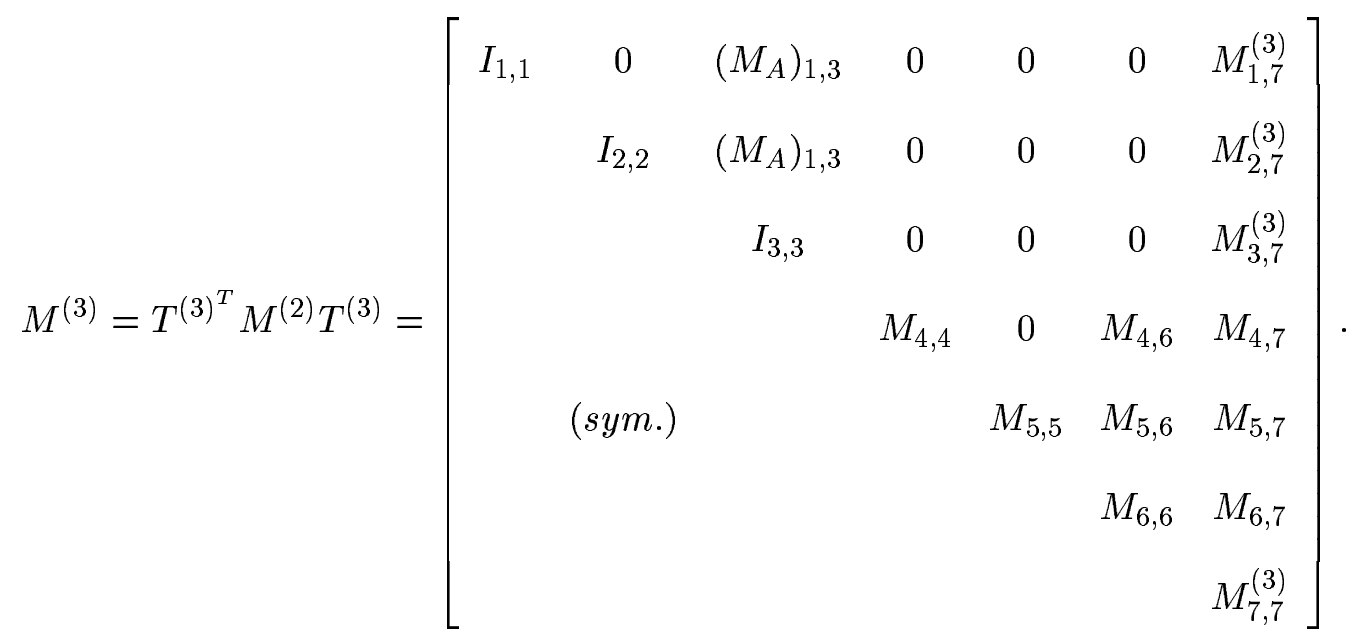


The transformed $K_{j}$ matrix is

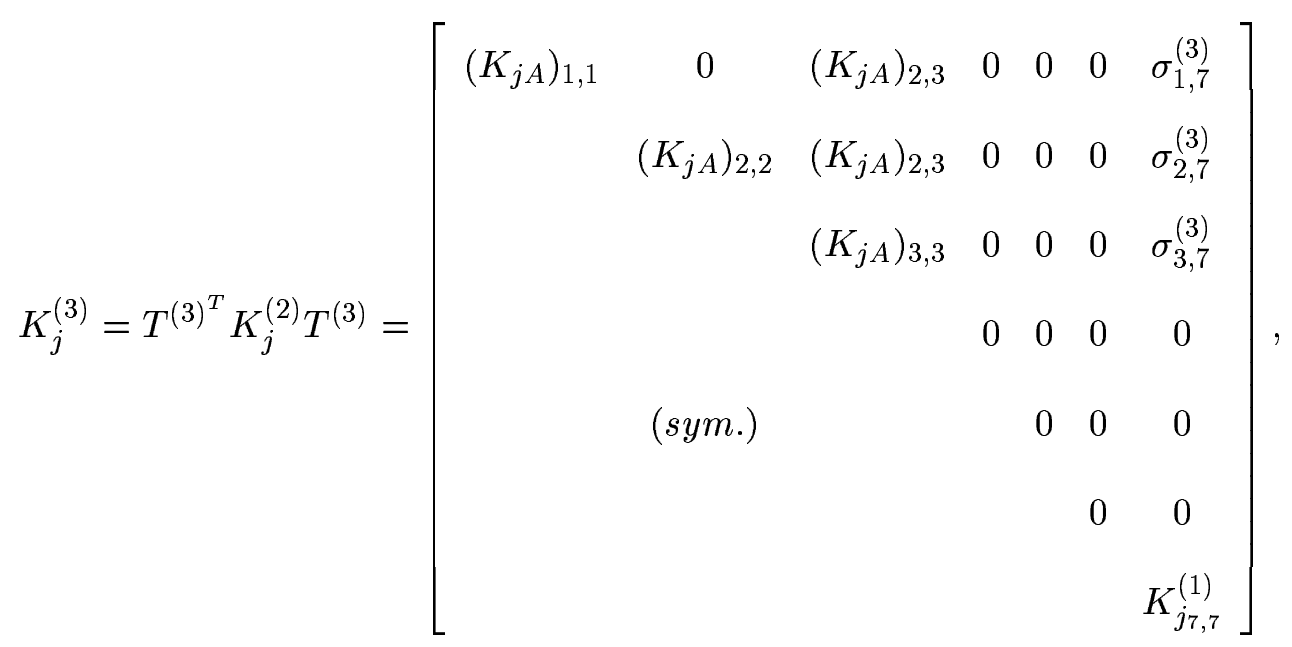

and the changed components of $K^{(3)}, M^{(3)}$, and $K_{j}^{(3)}$ are

$$
\begin{aligned}
K_{7,7}^{(3)} & =K_{(7)}^{(2)}+K_{(3,7)}^{(2)^{T}} \Psi_{(3,7)}, \\
M_{7,7}^{(3)} & =\Psi_{3,7}^{T}\left(M_{3,3}^{(2)} \Psi_{3,7}+M_{3,7}^{(2)}\right)+M_{3,7}^{(2)^{T}} \Psi_{3,7}+M_{7,7}^{(2)}, \\
M_{3,7}^{(3)} & =\Phi_{3,3}^{T}\left(M_{3,3}^{(2)} \Psi_{3,7}+M_{3,7}^{(2)}\right), \\
\left(M_{A}\right)_{i, 3} & =M_{1,3}^{(i)} \Phi_{3,3} \quad \text { for } i=1,2, \\
M_{i, 7}^{(3)} & =M_{i, 7}^{(i)}+M_{i, 7}^{(i)} \Psi_{3,7} \quad \text { for } i=1,2, \\
K_{j_{7,7}}^{(3)} & =\Psi_{3,7}^{T}\left(K_{j_{3,3}}^{(2)} \Psi_{3,7}+K_{j_{3,7}}^{(2)}\right)+K_{j_{3,7}}^{(2)^{T}} \Psi_{3,7}+K_{j_{7,7}}^{(2)}, \\
\sigma_{3,7}^{(3)} & =\Phi_{3,3}^{T}\left(K_{j_{3,3}}^{(2)} \Psi_{3,7}+K_{j_{3,7}}^{(2)}\right), \\
\left(K_{j A}\right)_{i, 3} & =\sigma_{1,3}^{(i)} \Phi_{3,3} \quad \text { for } i=1,2, \text { and } \\
\sigma_{i, 7}^{(3)} & =\sigma_{i, 7}^{(i)}+\sigma_{i, 7}^{(i)} \Psi_{3,7} \quad \text { for } i=1,2 .
\end{aligned}
$$

The submatrices $\left(M_{A}\right)_{1,3},\left(M_{A}\right)_{2,3},\left(K_{j A}\right)_{1,3}$, and $\left(K_{j A}\right)_{2,3}$ are fully transformed. 
The transformations for substructures 4 and 5 are similar to the transformation for substructures 1 and 2. The transformation for substructure 6 is similar to the transformation for substructure 3 .

The transformation of substructure 7 is given by

$$
T^{(7)}=\left[\begin{array}{ccccccc}
I_{1,1} & 0 & 0 & 0 & 0 & 0 & 0 \\
0 & I_{2,2} & 0 & 0 & 0 & 0 & 0 \\
0 & 0 & I_{3,3} & 0 & 0 & 0 & 0 \\
0 & 0 & 0 & I_{4,4} & 0 & 0 & 0 \\
0 & 0 & 0 & 0 & I_{5,5} & 0 & 0 \\
0 & 0 & 0 & 0 & 0 & I_{6,6} & 0 \\
0 & 0 & 0 & 0 & 0 & 0 & \Phi_{7,7}
\end{array}\right] .
$$

Application of $T^{(7)}$ to $K^{(6)}$ yields the fully transformed stiffness matrix

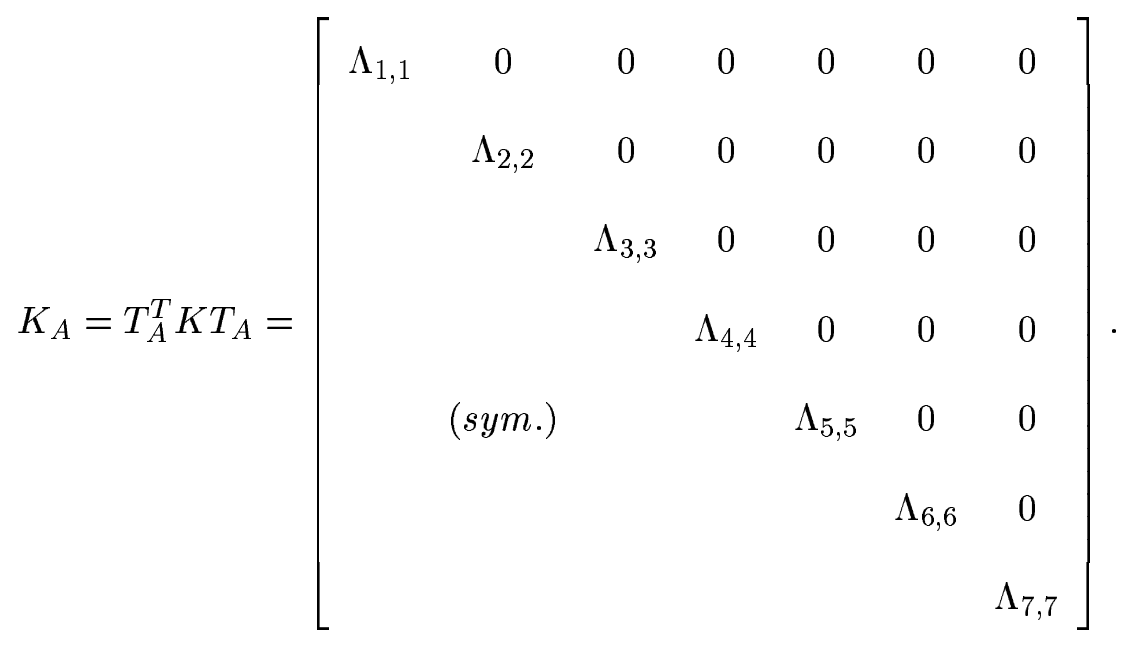


The fully transformed mass matrix $M_{A}$ in the AMLS subspace becomes

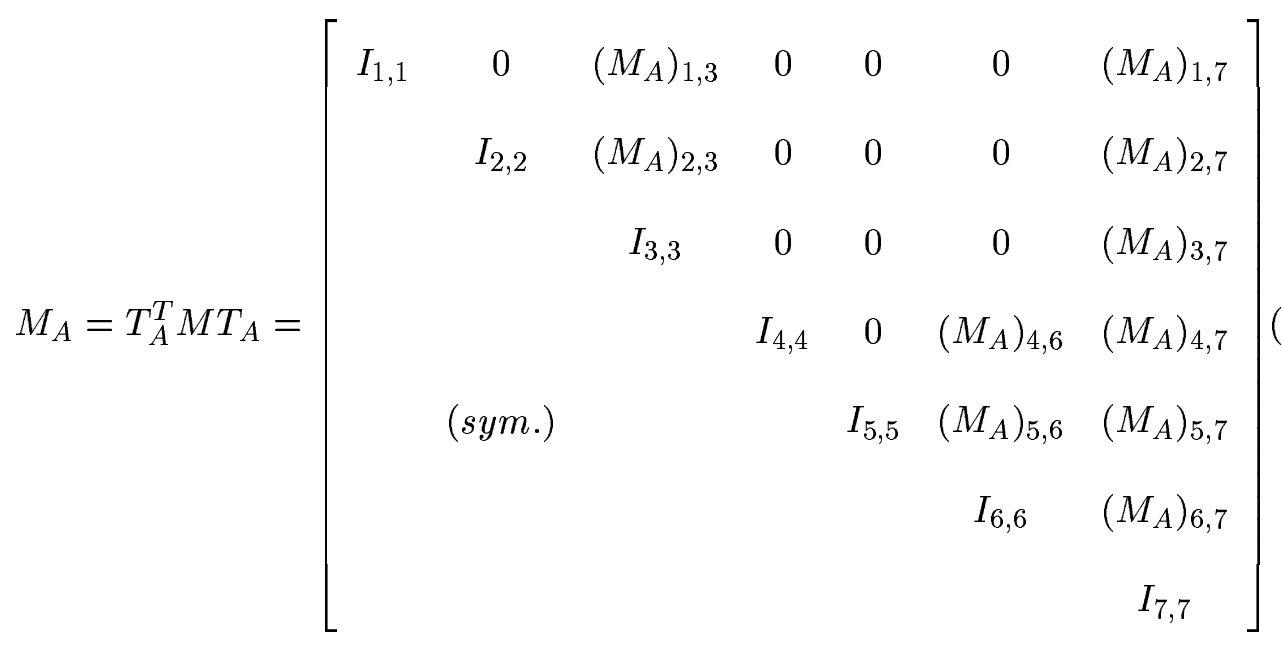

where the fully transformed mass submatrices $\left(M_{A}\right)_{i, 7}$ may be expressed as

$$
\left(M_{A}\right)_{i, 7}=M_{i, 7}^{(6)} \Phi_{7,7}, \quad \text { for } i=1,2, \ldots, 6
$$

The $K_{j}$ matrix in the AMLS subspace, $K_{j A}=T_{A}^{T} K_{j} T_{A}$, becomes

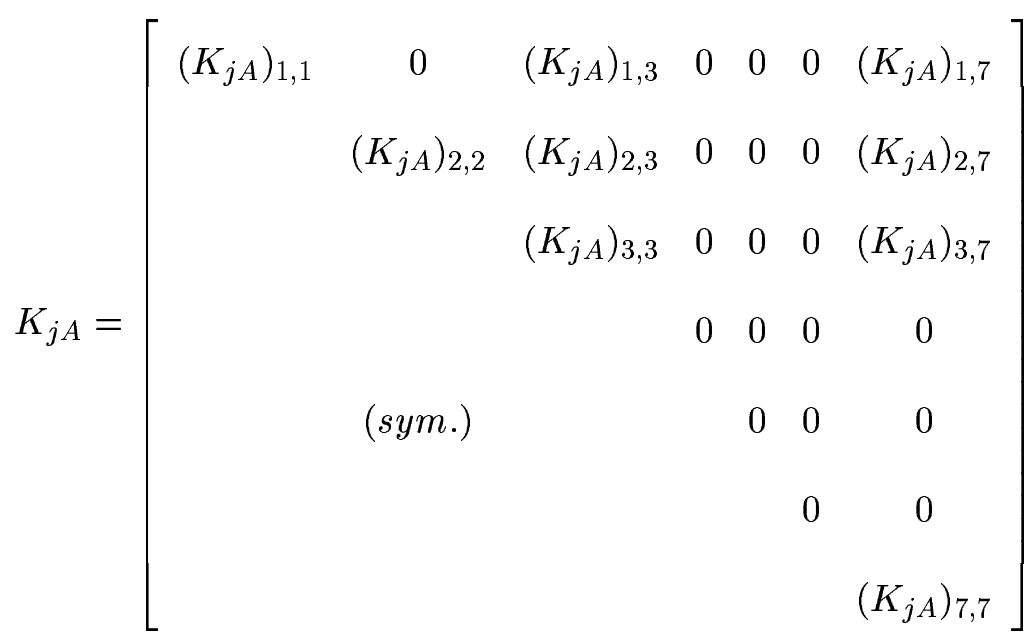

where the fully transformed mass submatrices $\left(K_{j A}\right)_{i, 7}$ may be expressed as

$$
\left(K_{j A}\right)_{i, 7}=\sigma_{i, 7}^{(6)} \Phi_{7,7}, \quad \text { for } i=1,2, \ldots, 6
$$


Finally, the transformation matrix $T_{A}$ is the overall transformation matrix and can be expressed as

$$
\begin{aligned}
T_{A}= & T^{(1)} T^{(2)} T^{(3)} \cdots T^{(7)} \\
T_{A}= & {\left[\begin{array}{ccccccc}
\Phi_{1,1} & 0 & \hat{\Psi}_{1,3} \Phi_{3,3} & 0 & 0 & 0 & \hat{\Psi}_{1,7} \Phi_{7,7} \\
0 & \Phi_{2,2} & \hat{\Psi}_{2,3} \Phi_{3,3} & 0 & 0 & 0 & \hat{\Psi}_{2,7} \Phi_{7,7} \\
0 & 0 & \Phi_{3,3} & 0 & 0 & 0 & \hat{\Psi}_{3,7} \Phi_{7,7} \\
0 & 0 & 0 & \Phi_{4,4} & 0 & \hat{\Psi}_{4,6} \Phi_{6,6} & \hat{\Psi}_{4,7} \Phi_{7,7} \\
0 & 0 & 0 & 0 & \Phi_{5,5} & \hat{\Psi}_{5,6} \Phi_{6,6} & \hat{\Psi}_{5,7} \Phi_{7,7} \\
0 & 0 & 0 & 0 & 0 & \Phi_{6,6} & \hat{\Psi}_{6,7} \Phi_{7,7} \\
0 & 0 & 0 & 0 & 0 & 0 & \Phi_{7,7}
\end{array}\right] }
\end{aligned}
$$

where $\hat{\Psi}_{i, j}$ may be expressed as

$$
\hat{\Psi}_{i, j}=\Psi_{i, j}+\sum_{k \in S_{i, j}} \Psi_{i, k} \hat{\Psi}_{k, j}
$$

where $S_{i, j}$ is the set of indices for ancestors of substructure $i$ that are the descendants of substructure $j$. Every $\hat{\Psi}_{k, j}$, in Eq. (5.28), must be calculated before $\hat{\Psi}_{i, j}$. Substituting this for the individual entries in $T_{A}$ yields the recursive relation

$$
\left(T_{A}\right)_{i, j}=\left\{\begin{array}{cc}
\Phi_{j} & \text { if } i=j \\
\hat{\Psi}_{i, j} \Phi_{j} & \text { if } i \in \mathcal{C}_{j} \\
0 & \text { otherwise }
\end{array}\right.
$$

where $\mathcal{C}_{j}$ is the set of indices for descendants of substructure $j$. 


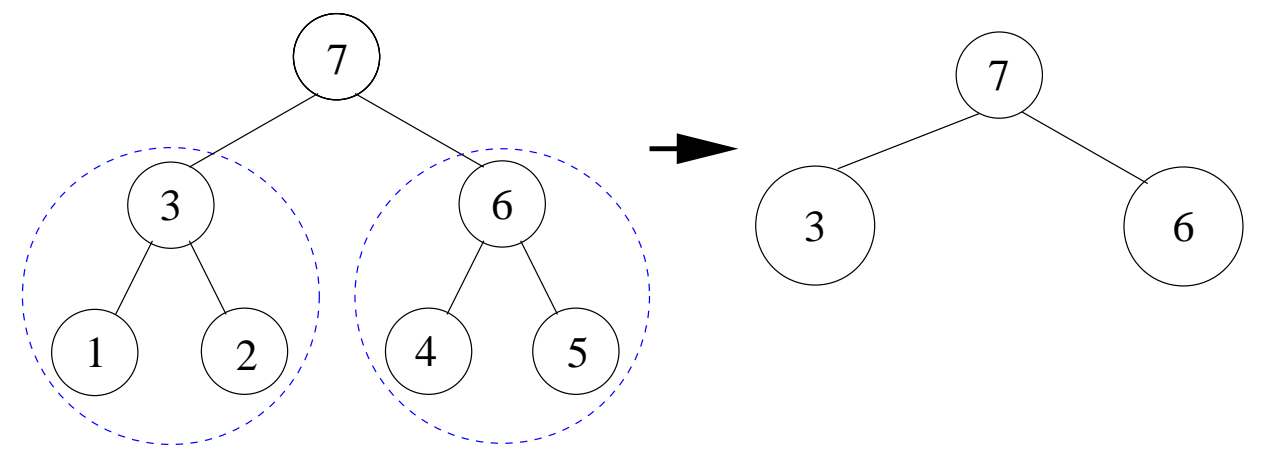

Figure 5.6: Subtree Diagram

\subsection{Phase 4: Generating the Distilled Subspace}

The first step in Phase 4 is a reduction technique called "distillation", which will be useful for FRPs also, where the known matrix sparsity structure in the $\mathcal{A}$ subspace lends itself to forming subtrees over which further reduction can be done. In the $\mathcal{A}$ subspace, $K_{A}$ is diagonal, $M_{A}$ has identity blocks on the diagonal and non-zero offdiagonal blocks, and the $K_{j}$ matrices have non-zero blocks on and off the diagonal. The $\mathcal{D}$ subspace is obtained by combining together substructures to create subtrees in the substructure tree, solving subtree eigenproblems, and truncating subtree and branch substructure eigenspaces [27]. This distillation process is best explained by the following example.

For the model plate problem, Fig. 5.6 shows substructures 1, 2, and 3 and substructures 4, 5, and 6 being combined to create subtrees 3 and 6 , respectively. The stiffness matrix $K_{A}$ is partitioned as 


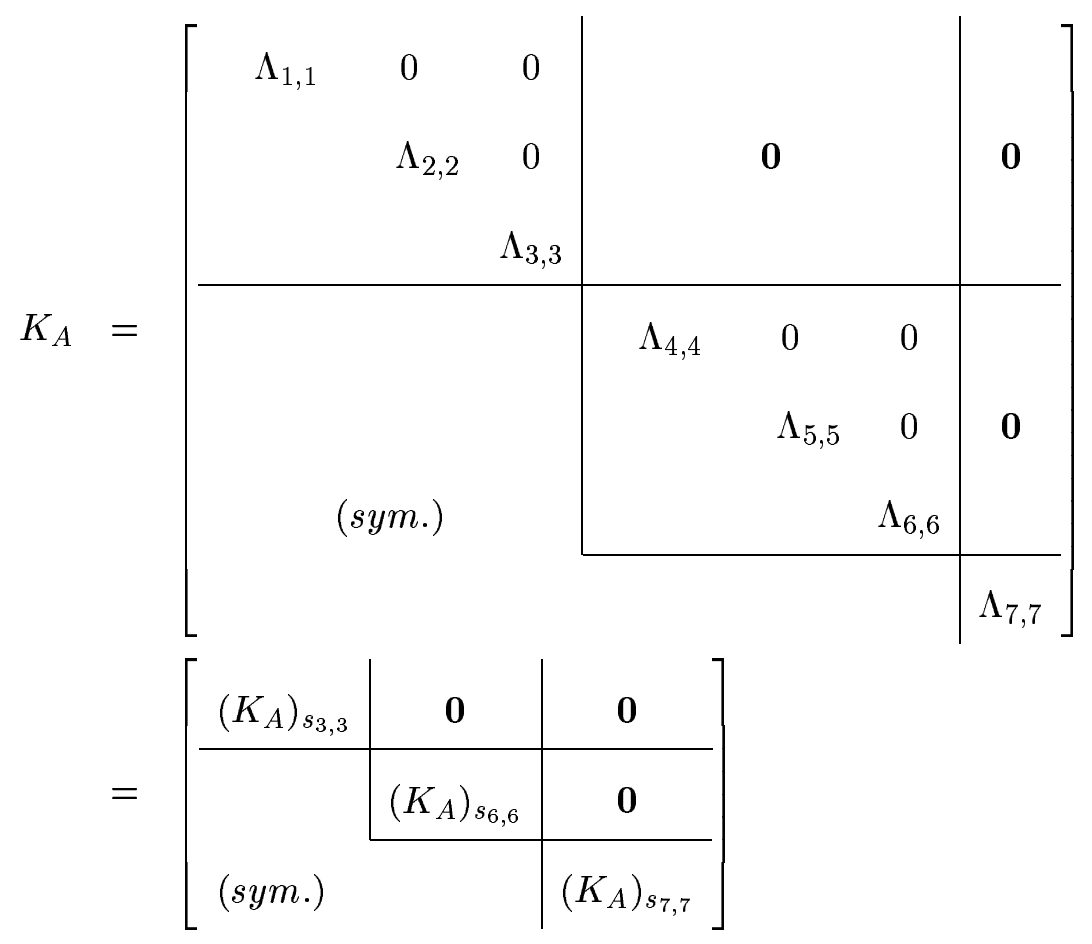

where $\left(K_{A}\right)_{s_{3,3}}$ is the subtree 3 stiffness matrix and likewise for subtree 6 . The mass matrix, $M_{A}$, is partitioned as

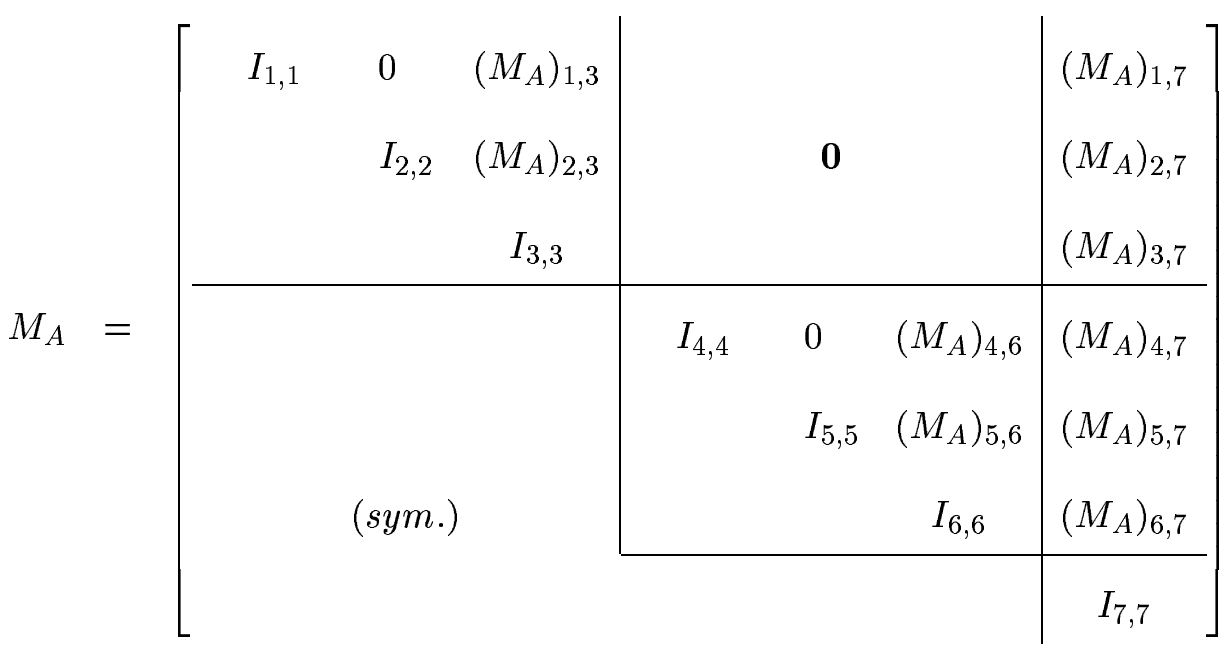


which can be written in the form

$$
M_{A}=\left[\begin{array}{c|c|c}
\left(M_{A}\right)_{s_{3,3}} & \mathbf{0} & \left(M_{A}\right)_{s_{3,7}} \\
\hline & \left(M_{A}\right)_{s_{6,6}} & \left(M_{A}\right)_{s_{6,7}} \\
\hline \text { sym. }) & & \left(M_{A}\right)_{s_{7,7}}
\end{array}\right] .
$$

The dimensions of submatrices $\left(K_{A}\right)_{s_{3,3}}$ and $\left(K_{A}\right)_{s_{6,6}}$ are the total number of substructure eigenvectors for subtrees 3 and 6 , respectively. In general, to form a subtree we accumulate substructures by moving the subtree root toward the root of the whole tree until moving it one level closer would cause the subtree size to exceed a maximum subtree size. The subscript, $s_{i, j}$, denotes the coupling between the substructure $i$ and substructure $j$. Only the $M_{A}$ and $K_{A}$ matrices are transformed to the $\mathcal{D}$ subspace in Phase 4 , and all of the $K_{j A}$ matrices are transformed in Phase $4 \mathrm{~d}$.

The eigenvalue problem for each subtree is

$$
\left(K_{A}\right)_{s_{i, i}} \Phi_{s_{i, i}}=\left(M_{A}\right)_{s_{i, i}} \Phi_{s_{i, i}} \Lambda_{s_{i, i}}
$$

and a partial eigensolution for the subtrees is computed for eigenvalues up to the distillation cutoff frequency $\omega_{D}$. The accuracy of the global eigenpairs depends on the distillation cutoff frequency $\omega_{D}$ and the target size of the subtrees. The subtrees effectively become termination points of the substructure tree. For this example, substructures $1,2,4$, and 5 are leaf substructures before distillation and subtrees 3 and 6 effectively become leaf substructures after distillation. For "branch substructures", such as substructure 7, we do not need to solve an eigenvalue problem; 
instead we just decide which substructure eigenvectors to keep by truncating the eigenvectors whose eigenvalues are above $\omega_{D}^{2}$.

The transformation matrix from the $\mathcal{A}$ subspace to the $\mathcal{D}$ subspace for the example problem is

$$
T_{D}=\left[\begin{array}{ccc}
\Phi_{s_{3,3}} & 0 & 0 \\
0 & \Phi_{s_{6,6}} & 0 \\
0 & 0 & {\left[\begin{array}{c}
I \\
0
\end{array}\right]_{s_{7,7}}}
\end{array}\right]=\left[\begin{array}{ccc}
\Phi_{s_{3,3}} & 0 & 0 \\
0 & \Phi_{s_{6,6}} & 0 \\
0 & 0 & B_{s_{7,7}}
\end{array}\right]
$$

where $\Phi_{s_{i, i}}$ are rectangular matrices that contain subtree eigenvectors. The transformed stiffness matrix $K_{D}$ is given by

$$
\begin{aligned}
& K_{D}=T_{D}^{T} K_{A} T_{D}
\end{aligned}
$$

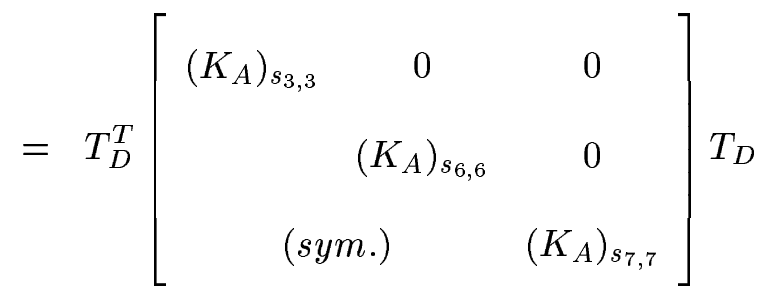

$$
\begin{aligned}
& =\left[\begin{array}{ccc}
\Lambda_{s_{3,3}} & 0 & 0 \\
\Lambda_{s_{6,6}} & 0 \\
\text { (sym. }) & \Lambda_{s_{7,7}}
\end{array}\right]
\end{aligned}
$$


Similarly, the transformed mass matrix $M_{D}$ is given by

$$
\begin{aligned}
M_{D} & =T_{D}^{T} M_{A} T_{D} \\
& =T_{D}^{T}\left[\begin{array}{rrr}
\left(M_{A}\right)_{s_{3,3}} & 0 & \left(M_{A}\right)_{s_{3,7}} \\
& \left(M_{A}\right)_{s_{6,6}} & \left(M_{A}\right)_{s_{6,7}} \\
& (\text { sym. }) & \left(M_{A}\right)_{s_{7,7}}
\end{array}\right] T_{D} \\
& =\left[\begin{array}{ccc}
I & \Phi_{s_{6,6}}^{T}\left(M_{A}\right)_{s_{7,7}} B_{s_{7,7}} \\
& I & \Phi_{s_{6,6}}^{T}\left(M_{A}\right)_{s_{7,7}} B_{s_{7,7}} \\
& &
\end{array}\right] .
\end{aligned}
$$

The properties of $M_{A}, K_{A}$, and $K_{j A}$ described at the beginning of this section are also retained by $M_{D}, K_{D}$, and $K_{j D}$.

\subsubsection{Computing the Eigenpairs}

Next, an approximate eigensolution of global eigenvalue problem

$$
K_{D} \Phi_{D}=M_{D} \Phi_{D} \Lambda_{D}
$$

is solved for those eigenpairs whose eigenvalues are less than $\omega_{g}^{2}$ where $\omega_{g}$ is the global cutoff frequency.

\subsubsection{Computing the Residual Flexibility Vectors}

To compensate for the contribution of higher frequency modes, static responses are computed by

$$
X_{s A}=\left[\operatorname{Re}\left\{K_{A}\left(\omega_{n}\right)\right\}\right]^{-1} F_{A}(0) .
$$


The matrix of static response vectors $X_{s A}$ is computed in the AMLS subspace because the cost of computing an inverse of the diagonal matrix $\operatorname{Re}\left\{K_{A}\left(\omega_{n}\right)\right\}$ is trivial.

\subsubsection{Computing the Damping Deformation Vectors}

The matrix of damping deformation vectors $X_{b A}$ is computed in the AMLS subspace from

$$
X_{b A}=\left[\operatorname{Re}\left\{K_{A}\left(\omega_{n}\right)\right\}\right]^{-1} F_{b A}
$$

where $F_{b A}$ is a matrix of force vectors created from replacing each viscous damping element by a pair of opposing forces. The computation of $X_{b A}$ is trivial for the same reason that $X_{s A}$ is.

\subsubsection{Orthogonalization of the RFVs and DDVs}

To eliminate linear dependence in the initial approximating subspace, the $X_{s A}$ and $X_{b A}$ matrices are orthogonalized against $\Phi_{A}$ and among themselves with respect to $\operatorname{Re}\left\{K_{A}\left(\omega_{n}\right)\right\}$. Because this process is identical for RFVs, DDVs, and DRVs, the explanation of this process is provided one time in Section 5.8.3 for the DRVs. In practice, it is necessary to repeat the orthogonalization process up to three times to ensure the vectors in the $X_{s A}$ and $X_{b A}$ matrices are orthogonal. The resulting product of $\Phi_{A}^{T} M_{A}\left[X_{s A} X_{b A}\right]$ is so close to null that it is ignored. 


\subsection{Phase 4d: Computing the Dynamic Response Vec- tors}

The purpose of Phase $4 \mathrm{~d}$ is to compute the DRVs on the $\mathcal{D}$ subspace, but first, the $K_{j A}$ and $F(\omega)$ matrices must be transformed to the $\mathcal{D}$ subspace.

\subsubsection{Projecting the Frequency Response Problem onto the Dis- tilled Subspace}

Continuing with the plate problem, the $K_{j A}$ matrices from Eq. (5.25) are partitioned as

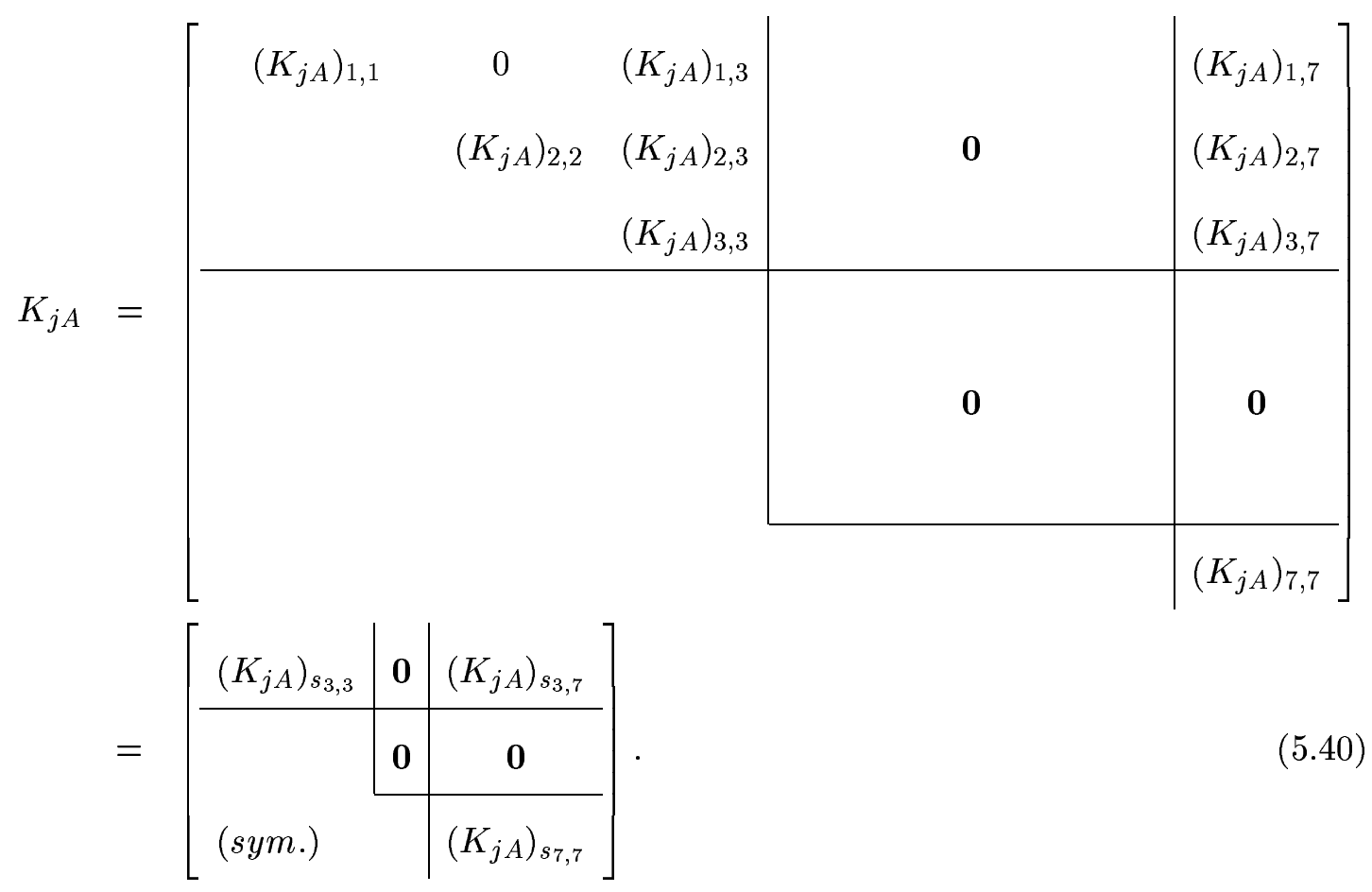

Because the $K_{j A}$ matrices only have nonzeros for substructures $1,2,3$ and 7 , the transformation process involves subtree 3, but not subtree 6, as shown in Fig. 5.7. 

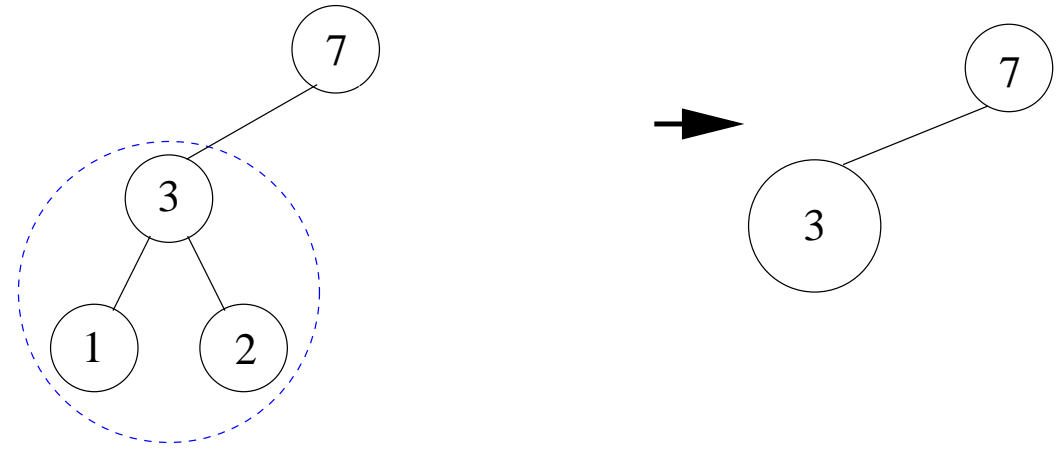

Figure 5.7: Substructure tree diagram of the distillation process for $K_{j D}$

The $K_{j A}$ matrices transformed to the $\mathcal{D}$ subspace are

$$
\begin{aligned}
K_{j D} & =T_{D}^{T} K_{j A} T_{D} \\
& =T_{D}^{T}\left[\begin{array}{ccc}
\left(K_{j A}\right)_{s_{3,3}} & 0 & \left(K_{j A}\right)_{s_{3,7}} \\
& 0 & 0
\end{array}\right] T_{D} \\
& \\
& =\left[\begin{array}{ccc}
\Phi_{s_{3,3}}^{T}\left(K_{j A}\right)_{s_{3,3}} \Phi_{s_{3,3}}^{T} & 0 & \Phi_{s_{6,6}}^{T}\left(K_{j A}\right)_{s_{7,7}} B_{s_{7,7}} \\
& 0 & 0 \\
(\text { sym. }) & & B_{s_{7,7}}\left(K_{j A}\right)_{s_{7,7}} B_{s_{7,7}}^{T}
\end{array}\right]
\end{aligned}
$$

where $T_{D}$ is from Eq. (5.34). The force matrix is transformed to the $\mathcal{D}$ subspace by

$$
F_{D}(\omega)=T_{D}^{T} T_{A}^{T} F(\omega)
$$

The last step is to transform the $B\left(\omega_{n}\right), B_{j}$, and $K_{s}\left(\omega_{n}\right)$ matrices to the $\mathcal{D}$ subspace by the same transformation process applied to the $K_{j}$ matrices. This 
transformation is defined by

$$
\begin{aligned}
B_{D}\left(\omega_{n}\right) & =T_{D}^{T} B_{A}\left(\omega_{n}\right) T_{D} \\
B_{j D} & =T_{D}^{T} B_{j A} T_{D}, \text { and } \\
K_{s D}\left(\omega_{n}\right) & =T_{D}^{T} K_{s A}\left(\omega_{n}\right) T_{D} .
\end{aligned}
$$

Finally, the interpolated FRP, Eq. (5.1), projected onto the $\mathcal{D}$ subspace is

$$
\begin{gathered}
{\left[-\omega^{2} M_{D}+(1+i \gamma) \operatorname{Re}\left\{K_{D}\left(\omega_{n}\right)\right\}+i \omega B_{D}\left(\omega_{n}\right)+i K_{s D}\left(\omega_{n}\right)\right.} \\
\left.+i \omega \sum_{j=1}^{p} h_{j}(\omega) B_{j D}+\sum_{j=1}^{q} g_{j}(\omega) K_{j D}\right] X_{r D}(\omega)=F_{D}(\omega)
\end{gathered}
$$

where $X(\omega)=T_{A} T_{D} X_{r D}(\omega)$.

\subsubsection{Computing Dynamic Response Vectors}

Computing DRVs on the $\mathcal{D}$ subspace from Eq. (5.44) can yield approximate DRVs that are close to the FE DRVs. The cost of computing DRVs on the $\mathcal{D}$ subspace can be significantly reduced if we first approximate the DRVs on a much smaller subspace called the initial approximating subspace $\mathcal{W}_{0}$. The cost of solving the FRP on the $\mathcal{W}_{0}$ subspace is significantly less because the dimension of $\mathcal{W}_{0}$ is about one-fourth the dimension of the $\mathcal{D}$ subspace, and the cost of computing a solution is on the order of the dimension cubed because the matrices are almost always fully populated.

The system matrices projected onto the initial approximating subspace $\mathcal{W}_{0 D}$ 
in the $\mathcal{D}$ subspace are given by

$$
\begin{aligned}
I & =W_{0 D}^{T} M_{D} W_{0 D} \\
\Lambda\left(\omega_{n}\right) & =W_{0 D}^{T} \operatorname{Re}\left\{K_{D}\left(\omega_{n}\right)\right\} W_{0 D}, \\
B_{W_{0}}\left(\omega_{n}\right) & =W_{0 D}^{T} B_{D}\left(\omega_{n}\right) W_{0 D}, \\
B_{j W_{0}} & =W_{0 D}^{T} B_{j D} W_{0 D}, \\
K_{s W_{0}}\left(\omega_{n}\right) & =W_{0 D}^{T} K_{s D}\left(\omega_{n}\right) W_{0 D}, \text { and } \\
K_{j W_{0}} & =W_{0 D}^{T} K_{j D} W_{0 D} .
\end{aligned}
$$

where $I$ is an identity matrix, $\Lambda$ is a diagonal matrix with the eigenvalues on the diagonal, and $W_{0 D}=\left[\Phi_{D} X_{s D} X_{b D}\right]$. The dimensions of all the matrices projected onto the $\mathcal{W}_{0}$ subspace in Eq. (5.45) are $n_{W_{0}}$ by $n_{W_{0}}$ where $n_{W_{0}}$ is the combined number of eigenvectors, RFVs, and DDVs.

Because of truncation of the eigenspace, the approximate solutions are typically inaccurate between $0.8 \omega_{\max }$ and $\omega_{\max }$. Therefore, we equally space $n_{\text {freq }} \mathrm{DRV}$ sampling frequencies in the frequency range $0.8 \omega_{\max }$ to $\omega_{\max }$. We recognize that this initial approach of selecting frequencies is somewhat arbitrary, and that a more efficient strategy would probably select sampling frequencies for DRVs adaptively based on the error in the frequency response at different frequencies. In the next section, we will only discuss how we compute DRVs at the $k^{\text {th }}$ sampling frequency, where $1 \leq k \leq n_{\text {freq }}$.

First, the force matrix at the $k^{t h}$ frequency is projected onto the initial 
approximating subspace by

$$
F_{W_{0}}\left(\omega_{k}\right)=W_{0 D}^{T} F_{D}\left(\omega_{k}\right)
$$

The dimensions of $F_{W_{0}}\left(\omega_{k}\right)$ are $n_{W_{0}}$ by $n_{L}$, where $n_{L}$ is the number of load cases. The modal solutions $Z\left(\omega_{k}\right)$ are computed from

$$
\begin{gathered}
{\left[-\omega_{k}^{2} I+(1+i \gamma) \Lambda\left(\omega_{n}\right)+i \omega_{k} B_{W_{0}}\left(\omega_{n}\right)+i K_{s W_{0}}\left(\omega_{n}\right)\right.} \\
\left.+i \omega_{k} \sum_{j=1}^{p} h_{j}\left(\omega_{k}\right) B_{j W_{0}}+\sum_{j=1}^{q} g_{j}\left(\omega_{k}\right) K_{j W_{0}}\right] Z\left(\omega_{k}\right)=F_{W_{0}}\left(\omega_{k}\right) .
\end{gathered}
$$

The dimensions of the solution matrix $Z\left(\omega_{k}\right)$ are $n_{W_{0}}$ by $n_{L}$. The solution matrix $Z\left(\omega_{k}\right)$ is then backtransformed from the initial approximating subspace to the $\mathcal{D}$ subspace by

$$
Y_{r D}\left(\omega_{k}\right)=W_{0 D} Z\left(\omega_{k}\right)
$$

The dimensions of $Y_{r D}\left(\omega_{k}\right)$ are $n_{D}$ by $n_{L}$ where $n_{D}$ is the dimension of the $\mathcal{D}$ subspace.

A matrix of residual vectors is computed from

$$
R_{D}\left(\omega_{k}\right)=A_{D}\left(\omega_{k}\right) Y_{r D}\left(\omega_{k}\right)-F_{D}\left(\omega_{k}\right)
$$

where the dimensions of $R_{D}\left(\omega_{k}\right)$ are $n_{D}$ by $n_{L}$ and the coefficient matrix $A_{D}\left(\omega_{k}\right)$ is defined as

$$
\begin{gathered}
A_{D}\left(\omega_{k}\right)=-\omega_{k}^{2} M_{D}+(1+i \gamma) \operatorname{Re}\left\{K_{D}\left(\omega_{n}\right)\right\}+i \omega_{k} B_{D}\left(\omega_{n}\right)+i K_{s D}\left(\omega_{n}\right) \\
+i \omega_{k} \sum_{j=1}^{p} h_{j}\left(\omega_{k}\right) B_{j D}+\sum_{j=1}^{q} g_{j}\left(\omega_{k}\right) K_{j D}
\end{gathered}
$$


A seminorm related to potential energy is computed for each of the $n_{L}$ residual vectors $r_{D}\left(\omega_{k}\right)$ in $R_{D}\left(\omega_{k}\right)$ to determine whether a DRV should be approximated from the residual $R_{D}\left(\omega_{k}\right)$ to enrich the approximating subspace. The energy seminorm of a residual vector is defined as

$$
\left\|r_{D}\left(\omega_{k}\right)\right\|_{K_{D}} \equiv\left[\left(\bar{r}_{D}\left(\omega_{k}\right)\right)^{T} \operatorname{Re}\left\{K_{D}\left(\omega_{n}\right)\right\}^{+} r_{D}\left(\omega_{k}\right)\right]^{1 / 2}
$$

where the overbar represents the complex conjugate. Extremely small nonzero values in diagonal matrix $\operatorname{Re}\left\{K_{D}\left(\omega_{n}\right)\right\}$ are replaced with zeros before computing the pseudoinverse in Eq. (5.51). For evaluating whether a DRV should be computed, the ratio $\left\|r_{D}\left(\omega_{k}\right)\right\|_{K_{D}} /\left\|f_{D}\left(\omega_{k}\right)\right\|_{K_{D}}$ is formed, where $f_{D}\left(\omega_{k}\right)$ is the column of matrix $F_{D}\left(\omega_{k}\right)$ corresponding to $r_{D}\left(\omega_{k}\right)$.

If the energy seminorm ratios of all of the $n_{L}$ residuals are below a user defined cutoff $\epsilon$, then no DRVs are approximated at that frequency. If any of the residuals' energy seminorm ratios are above $\epsilon$, a DRV is approximated from that residual. Simply stated, DRVs will only be computed from the residuals whose energy seminorm ratio is large enough to indicate that the approximating subspace would benefit from enrichment.

DRVs can be accurately computed from

$$
X_{r D}\left(\omega_{k}\right)=\left[A_{D}\left(\omega_{k}\right)\right]^{-1} R_{D}\left(\omega_{k}\right)
$$

or inexpensively approximated from

$$
X_{r D}\left(\omega_{k}\right)=\left[\operatorname{diag}\left\{A_{D}\left(\omega_{k}\right)\right\}\right]^{+} R_{D}\left(\omega_{k}\right) .
$$


In Eq. (5.53), only the diagonal of the coefficient matrix $A_{D}\left(\omega_{k}\right)$ is used in computing the pseudo-inverse. The dimensions of $X_{r D}\left(\omega_{k}\right)$ are $n_{D}$ by $n_{L 2}$ where $n_{L 2}$ is the number of residuals whose energy seminorm ratio is above $\epsilon$. In the next chapter, we compare DRVs computed from Eq. (5.52) and Eq. (5.53) by comparing approximate FRP solutions.

Because the matrix of DRVs $X_{r D}\left(\omega_{k}\right)$ is complex-valued and the approximating subspace only contains real-valued vectors, the real and imaginary components of the DRVs are saved as separate real-valued vectors. The last step is to transform the matrix of DRVs $X_{r D}\left(\omega_{k}\right)$ from the $\mathcal{D}$ subspace to the $\mathcal{A}$ subspace by

$$
X_{r A}\left(\omega_{k}\right)=T_{D} X_{r D}\left(\omega_{k}\right)
$$

where the dimensions of $X_{r A}$ are $n_{A}$ by $2 n_{L 2}$ for the $k^{\text {th }}$ frequency.

The process of computing DRVs starting from Eq. (5.46) through Eq. (5.54) is repeated for all $n_{f r e q}$ frequencies. Next, the matrix of DRVs $X_{r A}$ will be included in the approximating subspace after the following steps.

\subsubsection{Orthogonalization of the Dynamic Response Vectors}

To eliminate linear dependence, the DRVs are orthogonalized against the vectors contained in the initial approximating subspace and among themselves with respect to $\operatorname{Re}\left\{K_{A}\left(\omega_{n}\right)\right\}$. The orthogonalization process is completed on the $\mathcal{A}$ subspace using the block modified Gram-Schmidt algorithm (bMGS) by Björck [19]. The vectors 
of the $X_{r A}$ matrix are orthogonalized against $W_{0 A}$ with respect to $\operatorname{Re}\left\{K_{A}\left(\omega_{n}\right)\right\}$ as

$$
\left[X_{r A}\right] \leftarrow \prod_{j=1}^{n b}\left(I-W_{A 0 j} U_{j} W_{A 0 j}^{T} \operatorname{Re}\left\{K_{A}\left(\omega_{n}\right)\right\}\right)\left[X_{r A}\right]
$$

where $W_{0 A}=\left[\Phi_{A} X_{s A} X_{b A}\right]$ is partitioned into $n b$ column blocks, so $W_{A 0 j}$ is the $j^{\text {th }}$ column block of the matrix $W$. The details of how $U_{j}$ is formed are explained by $\operatorname{Kim}[27]$.

\subsubsection{Solving the Small Eigenvalue Problem}

The DRVs are orthonormalized with respect to $M_{A}$ by solving a small EVP defined by

$$
K_{X} Q_{X}=M_{X} Q_{X} \Lambda_{X}
$$

where $K_{X}=X_{r A}^{T} \operatorname{Re}\left\{K_{A}\left(\omega_{n}\right)\right\} X_{r A}$ and $M_{X}=X_{r A}^{T} M_{A} X_{r A} . \quad \Lambda_{X} \in \mathbb{R}^{n_{x} \times n_{x}}$ is a diagonal matrix containing DRV eigenvalues, $Q_{X} \in \mathbb{R}^{n_{x} \times n_{x}}$ is a matrix containing the corresponding eigenvectors in its columns, and $n_{x}$ is the number of orthonormal DRVs. The $M_{A}$-orthonormalized DRVs and their corresponding eigenvalues are

$$
X_{r A}=X_{R} Q_{R} \quad \text { and } \quad \Lambda_{A}=\Lambda_{X}
$$

\subsubsection{Computation of Modal Mass Matrix}

We use the AMLS method to efficiently compute a truncated set of eigenvectors from a reduced eigenvalue problem so we can inexpensively solve a FRP. Using the AMLS method, we can compute eigenvectors in significantly less time than other 
competitive approaches [26]. After computing the eigenvectors, we orthogonalize the eigenvectors, DRVs, DDVs, and RFVs against each other with respect to the stiffness matrix using the previously explained bMGS method. This approach does not result in the eigenvectors being orthogonal to the DRVs, DDVs, and RFVs with respect to the mass matrix. Therefore, the modal mass matrix is

$$
M_{W}=W_{A}^{T} M_{A} W_{A}=\left[\begin{array}{cc}
I & \hat{M} \\
\hat{M}^{T} & I
\end{array}\right]
$$

where $\hat{M}=\left[\Phi_{A} X_{s A} X_{b A}\right]^{T} M_{A}\left[X_{r A}\right]$. The $\hat{M}$ matrix is inexpensive to compute and its existence does not increasse the cost of solving the modal FRP.

\subsection{Phase 5: Backtransformation to the FE space}

The original purpose of Phase 5 is to transform $\Phi_{A}$ from the $\mathcal{A}$ subspace to the $\mathcal{F}$ subspace. For this research, Phase 5 has been modified to transform $B_{A}\left(\omega_{n}\right), B_{j A}$, $K_{s A}\left(\omega_{n}\right)$, and, $K_{j A}$ from the $\mathcal{A}$ subspace to the approximating subspace $\mathcal{W}$. The transformation of all of the FE matrices from the $\mathcal{A}$ subspace to the $\mathcal{W}$ subspace 
can be written as

$$
\begin{aligned}
M_{W} & =\left[\begin{array}{cc}
I & \hat{M} \\
\hat{M}^{T} & I
\end{array}\right] \\
\Lambda\left(\omega_{n}\right) & =W_{A}^{T} K_{A}\left(\omega_{n}\right) W_{A} \\
B_{W}\left(\omega_{n}\right) & =W_{A}^{T} B_{A}\left(\omega_{n}\right) W_{A} \\
B_{j W} & =W_{A}^{T} B_{j A} W_{A} \\
K_{s W}\left(\omega_{n}\right) & =W_{A}^{T} K_{s A}\left(\omega_{n}\right) W_{A}, \text { and } \\
K_{j W} & =W_{A}^{T} K_{j A} W_{A} .
\end{aligned}
$$

\subsection{Phase 6d: Solving the FRP on the Approximating Subspace}

With all of the system matrices projected onto the approximating subspace, the FRP, Eq. (5.1), projected onto the approximating subspace is

$$
\begin{gathered}
{\left[-\omega^{2} M_{W}+(1+i \gamma) \Lambda\left(\omega_{n}\right)+i \omega B_{W}\left(\omega_{n}\right)+i K_{s W}\left(\omega_{n}\right)\right.} \\
\left.+i \omega \sum_{j=1}^{p} h_{j}(\omega) B_{j W}+\sum_{j=1}^{q} g_{j}(\omega) K_{j W}\right] Y_{W}(\omega)=F_{W}(\omega)
\end{gathered}
$$

where $X(\omega)=W Y_{W}(\omega)$. Solutions are computed at all frequencies of interest and are written to disk so the FE software package can backtransform the solution to FE coordinates. 


\section{Chapter 6}

\section{Numerical Results}

In this chapter, the efficiency and accuracy of the solution algorithm presented in this dissertation are evaluated on three finite element (FE) models that have viscoelastic materials (VEMs). The parameters that directly control the accuracy and performance of the solution algorithm are determined by comparing approximate solutions computed on different approximating subspaces. We start with an approximating subspace that only contains eigenvectors to evaluate the effect of the nominal frequency on solution accuracy. Next, we include enrichment vectors in the approximating subspace called residual flexibility vectors (RFVs) and damping deformation vectors (DDVs) to improve solution accuracy. At this point, the approximating subspace that includes eigenvectors, RFVs, and DDVs is called the initial approximating subspace $\left(\mathcal{W}_{0}\right)$. After the dynamic response vectors (DRVs) are included, the approximating subspace is denoted by $\mathcal{W}$. In the end, the goal 
is to compute solutions on a small enriched approximating subspace as quickly as possible with minimal loss of accuracy.

\subsection{Parameters}

Only those parameters that have a significant impact on the accuracy and efficiency of the computational approach are discussed here. These parameters are separated into two groups depending on their effect on the total elapsed time. The parameters of Group One are

1. the nominal frequency $\omega_{n}$,

2. the number of residual flexibility vectors (RFVs), and

3. the number of damping deformation vectors (DDVs).

The parameters of Group One have little to no impact on elapsed time, but choosing them poorly can undermine the final accuracy of the approximate solution.

The approximate solutions can still be inaccurate after the parameters of Group One have been optimized. Therefore, the parameters of Group Two must be modified to improve solution accuracy. Since the parameters of Group Two can have a significant impact on the total elapsed time and solution accuracy, the tradeoff between accuracy and elapsed time is carefully analyzed. The parameters in the Group Two are

1. the global cutoff frequency $\omega_{g}$, 


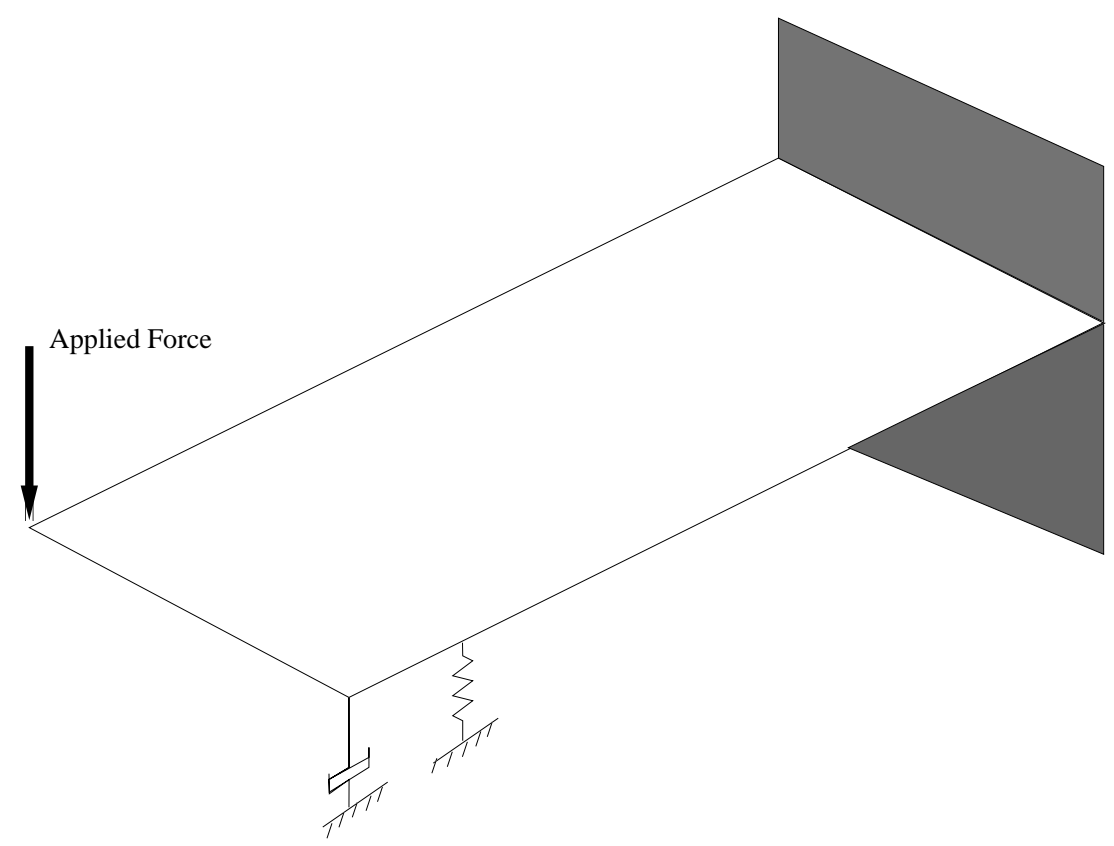

Figure 6.1: Cantilever plate model

2. the selection and type of dynamic response vectors (DRVs), and

3. the substructure cutoff frequency $\omega_{A}$.

The approach taken in this chapter is to evaluate and discuss the effects of each parameter starting from the least computationally expensive to the most. The parameters of Group One are discussed after the cantilever plate model is introduced.

\subsection{Cantilever Plate Model}

The cantilever plate model, as shown in Fig. 6.1, serves as an evaluation tool to demonstrate the effect of various parameters on the accuracy of the approximate solution. The cantilever plate is $900 \mathrm{~mm}$ long by $600 \mathrm{~mm}$ wide with a spring (100 


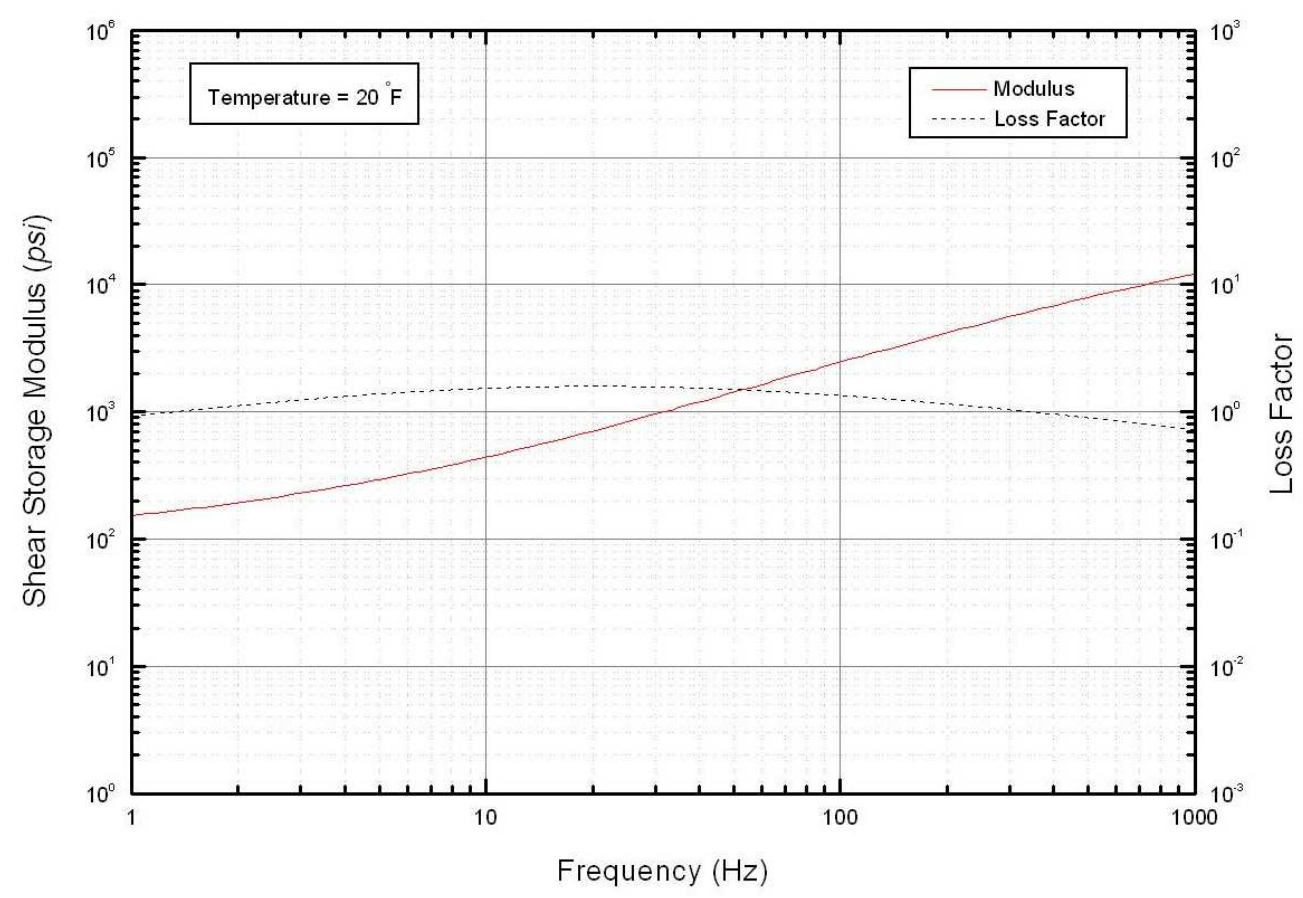

Figure 6.2: RA925 Properties

$\mathrm{N} / \mathrm{mm})$ and viscous damper $(10 \mathrm{Ns} / \mathrm{mm})$ attached near the edge of the free end of the plate. The entire cantilever plate is a constrained layer damping (CLD) sandwich of steel/VEM/steel of thickness $0.508 \mathrm{~mm} / 0.0508 \mathrm{~mm} / 0.508 \mathrm{~mm}$, respectively. The CLD sandwich is modeled using the "shell/volume/shell" FE modeling approach where the upper and lower elastic faces are modeled with shell elements and the VEM core is modeled with solid elements. The FE model is composed of 10,680 4-node shell elements and 5,340 8-node solid elements resulting in a 65,886 DOF FE model.

The Young's modulus for the upper and lower steel face sheets is 206,800 $\mathrm{N} / \mathrm{mm}^{2}$. The VEM core is called Dynalam RA-925 and is manufactured by Roush 
Industries who provided the shear modulus and loss factor data shown in Fig. 6.2. The shear modulus $G(\omega)$ is related to the Young's modulus $E(\omega)$ through the relationship $E(\omega)=2 G(\omega)(1-\nu)$ where Poisson's ratio $\nu$ is 0.485 for the VEM. The force input location is shown in Fig. 6.1, and the measurement location is the point on the plate where the viscous damper is attached, unless indicated otherwise.

The frequency range of interest for the frequency response analysis is from 0 to $800 \mathrm{~Hz}\left(\omega_{\min }=0.0\right.$ and $\left.\omega_{\max }=2 \pi \cdot 800.0 \mathrm{~Hz}\right)$. The global cutoff frequency $\omega_{g}$ is set to $1.1 \omega_{\max }$ for all computations until Section 6.4 .1 where the effects of varying $\omega_{g}$ are evaluated in terms of solution accuracy and elapsed time. From the example FRPs, we find that when $\omega_{g}$ is less than $1.1 \omega_{\max }$ the accuracy of the approximate solution decreases significantly because all modes near resonance are not included in the approximating subspace. The substructure mode cutoff frequency $\omega_{A}$ is set to $5.0 \omega_{g}$ until Section 6.4 .5 where the ratio $\omega_{A} / \omega_{g}$ is evaluated in terms of solution accuracy and elapsed time. The sampling frequencies for the interpolation approach are 1, 400, and $800 \mathrm{~Hz}$ and the interpolation functions are cubic splines for all FRPs in this chapter are defined in Section 4.1.

The direct solution of the interpolated FRP, Eq. (4.10), is represented as a solid red line in every FRF plot. Computing the direct solution at 800 frequencies takes 5 hours and 20 minutes on a single microprocessor. All approximate solutions presented in this chapter are computed from the reduced interpolated FRP, Eq. (5.60), and are represented by dashed lines of various colors in all FRF plots in this chapter. 


\subsection{Group One Parameters}

\subsubsection{Effect of the Nominal Frequency}

Figure 6.3 shows that solution accuracy increases for increasing values of the nominal frequency $\omega_{n}$. Interestingly, the approximate solution computed with $\omega_{n}$ equal to the highest excitation frequency $\omega_{\max }$ is consistently more accurate than the other solutions not just at frequencies near $\omega_{n}$, but at all frequencies. This is not too surprising, when we look more closely at what is happening in the approximation.

For the case $\omega_{n}=0.0$, the approximate solution is inaccurate because the parts of the stiffness matrix $\operatorname{Re}\left\{K\left(\omega_{n}\right)\right\}$ that represent the VEM core are generated with a shear modulus value from the rubbery or low frequency range (see Fig. 2.2) in which the VEM core has a relatively low shear modulus. For the eigenvectors of the global eigenvalue problem, Eq. (1.2), whose natural frequencies are greater than $\omega_{n}$, their natural frequencies are lower than they would be if they were computed with $\omega_{n}$ closer to their own values. If $\omega_{n}=\omega_{m a x}$, accuracy at the high end of the frequency range is better for that reason, and the accuracy at lower frequencies is retained because for them, the cutoff frequency is a higher multiple of the response frequency, so you have an abundant subspace of approximation and the truncation effects are less evident.

Figure 6.3 shows that the damping is exaggerated in the computed response for the case $\omega_{n}=0.0$, especially near $\omega_{\max }$. If the eigenvectors with the larger eigenvalues are computed with too low a value of $\omega_{n}$, the shear modulus of the VEM 

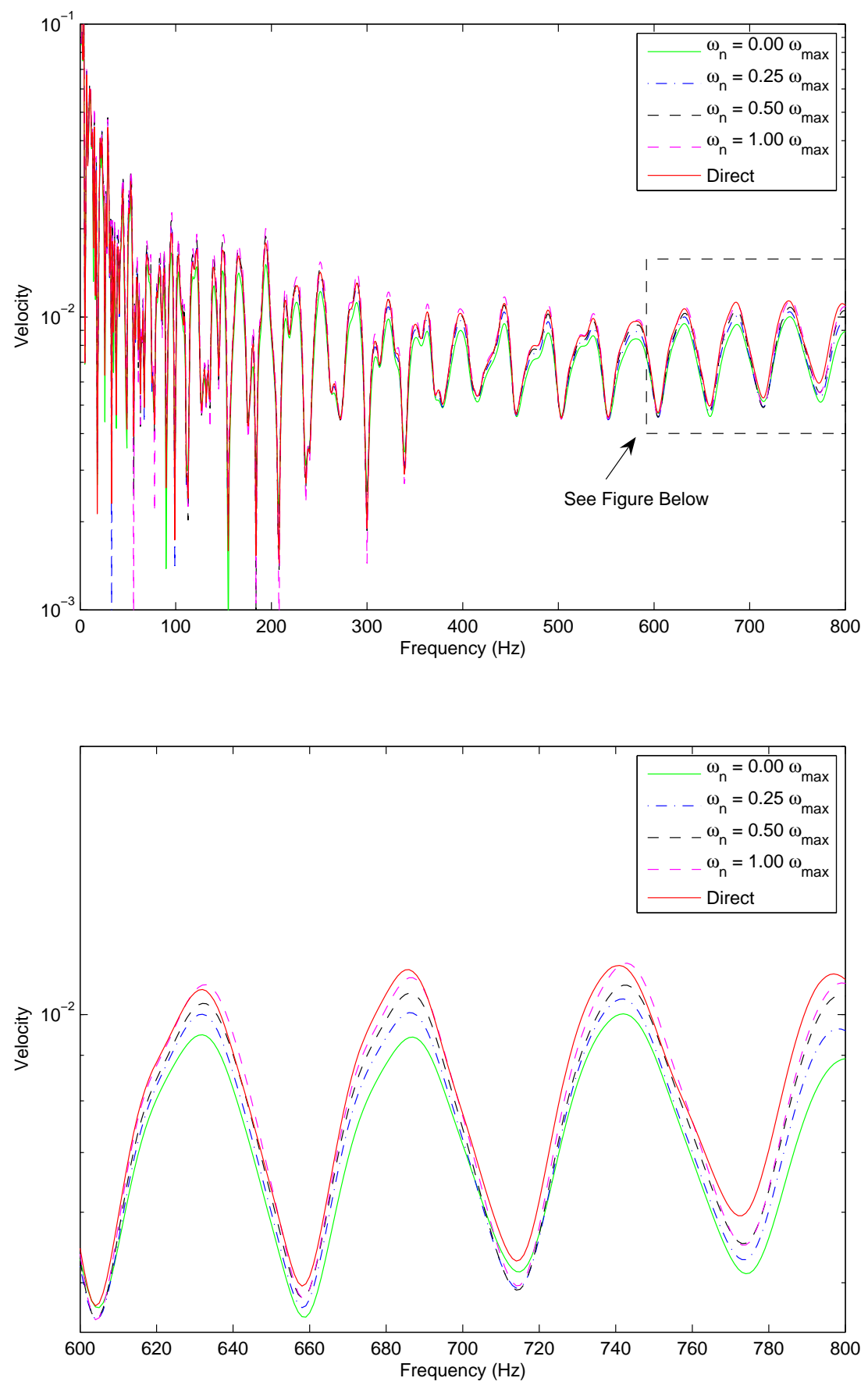

Figure 6.3: Effect of the nominal frequency $\omega_{n}$ for the cantilever plate model with inset in bottom graph 
will be too low, and the relative displacement between the upper and lower faces will be too great. Then, when the FRP is solved at higher frequencies, there will be too much shear deformation in the VEM and consequently too much dissipation of energy.

\subsubsection{Effect of the Residual Flexibility Vectors (RFVs)}

Figure 6.4 shows a dramatic increase in accuracy from including an RFV in the approximating subspace. This increase in solution accuracy is dramatic because the static response accounts for localized deformations and the eigenspace does not accurately represent the localized deformation. The FRFs in Fig. 6.4 are drivepoint FRFs, where the measurement and force input location are coincident, so the FRF shown in Fig. 6.4 has the most dramatic increase in solution accuracy. For the case of the cross-FRF, where the measurement and force input location are not coincident, Fig. 6.5 shows that the RFV's improvement in solution accuracy is negligible. Overall, RFVs increase solution accuracy at a very low computational cost and are almost always worth computing.

\subsubsection{Effect of the Damping Deformation Vectors (DDVs)}

Figure 6.5 shows that including a DDV in the approximating subspace improves solution accuracy. The number of DDVs is always equal to or less than the number of viscous dampers in the FE model, and for the cantilever plate model, we only com-

pute one DDV. The increase in solution accuracy is typically larger for FRPs where 

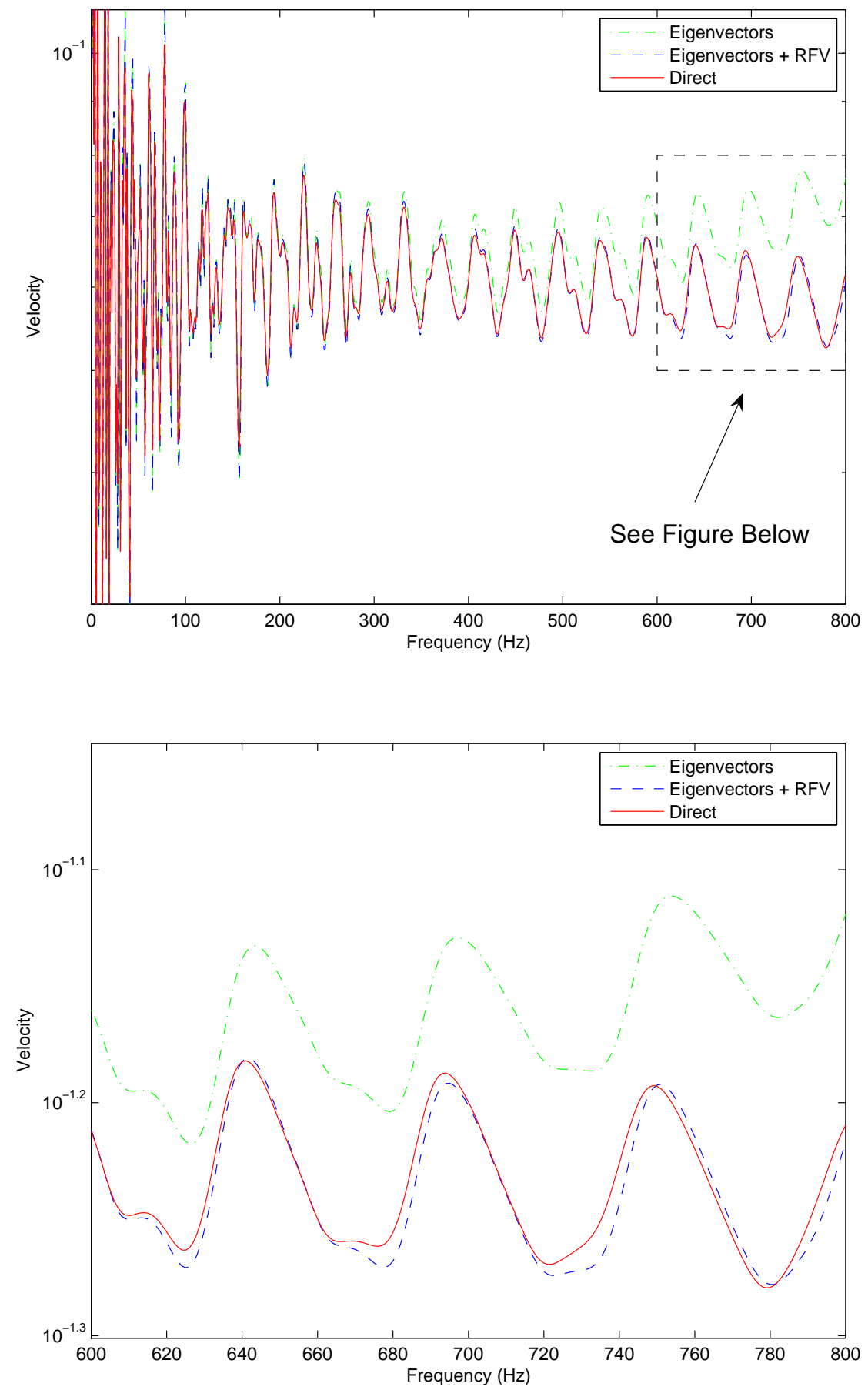

Figure 6.4: Effect of including a RFV in the approximating subspace on solution accuracy for the cantilever plate model with inset in bottom graph 

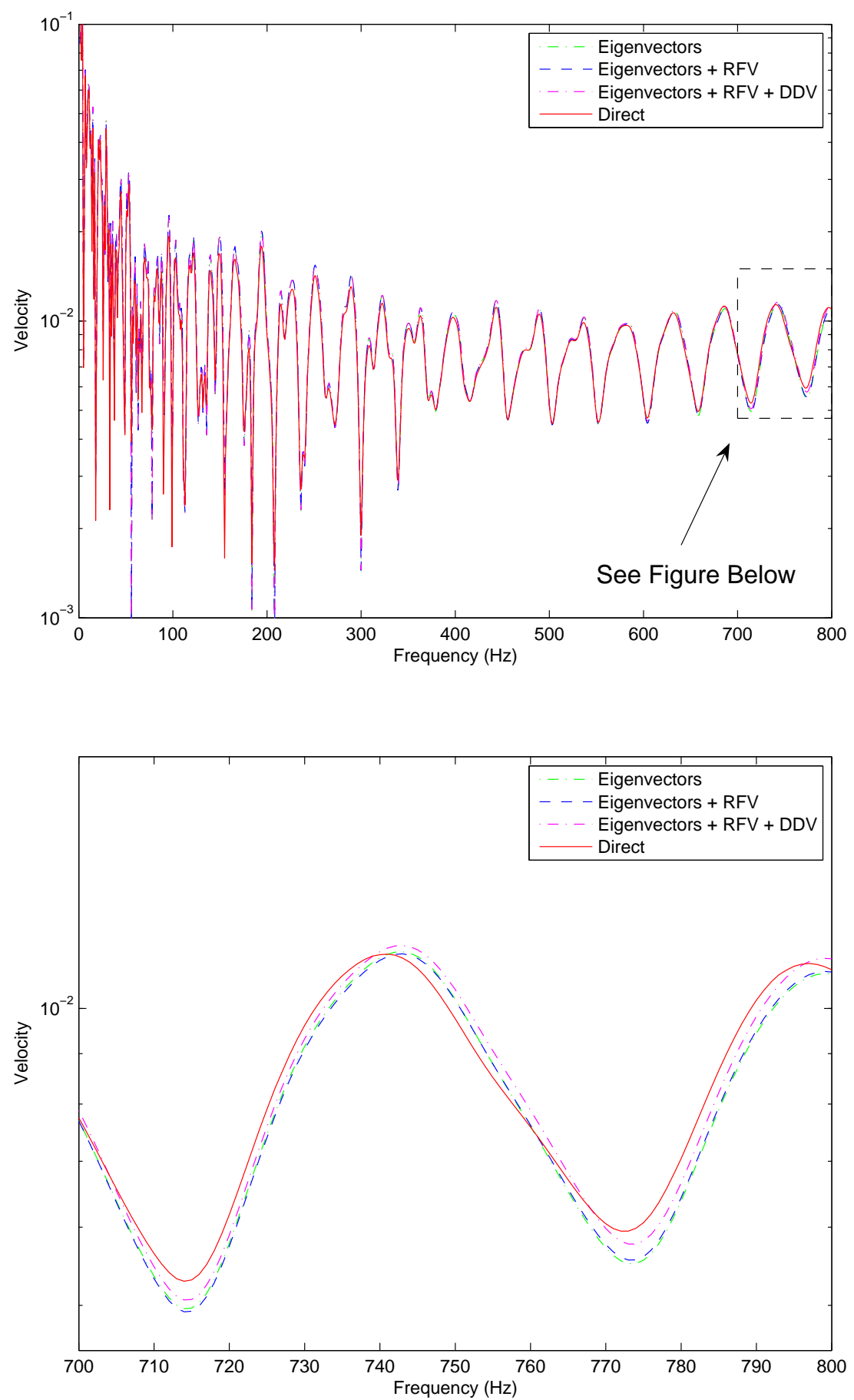

Figure 6.5: Effect of including a DDV in the approximating subspace on solution accuracy for the cantilever plate model with inset in bottom graph 
the measurement location is near the viscous damper because the viscous damper causes localized deformation in the structure. Like the RFVs, including DDVs in the approximating subspace can improve accuracy at a very low computational cost.

\subsection{Group Two Parameters}

The parameters of Group Two also increase solution accuracy but at a potentially high computational cost. The first two parameters of Group Two, the global cutoff frequency $\omega_{g}$ and the selection and type of DRVs, are explained next. The explanation of the last parameter of Group Two, substructure cutoff frequency $\omega_{A}$, follows the introduction of the quarter panel model.

\subsubsection{Effect of the Global Cutoff Frequency}

The number of eigenvectors computed from the global EVP, Eq. (1.2), depends on the global cutoff frequency $\omega_{g}$ because only the eigenvectors whose eigenvalues are less than $\omega_{g}^{2}$ are computed. The heuristic industry standard for $\omega_{g}$, when residual flexibility is used, is $1.5 \omega_{\max }$, where $\omega_{\max }$ is the highest excitation frequency. To be more efficient, we set $\omega_{g}=1.1 \omega_{\max }$ earlier in this chapter. Next, we will evaluate the effect of $\omega_{g}$ on solution accuracy and elapsed time.

Table 6.1 shows that increasing $\omega_{g}$ from $1.1 \omega_{\max }$ to $1.5 \omega_{\max }$ increases the total elapsed time $76.5 \%$ from 15 minutes and 16 seconds to 26 minutes and 57 seconds. This $76.5 \%$ increase in elapsed time is primarily due to the fact that the dimension of the approximating subspace increases $36.7 \%$ from 1,054 to 1,441 (see 

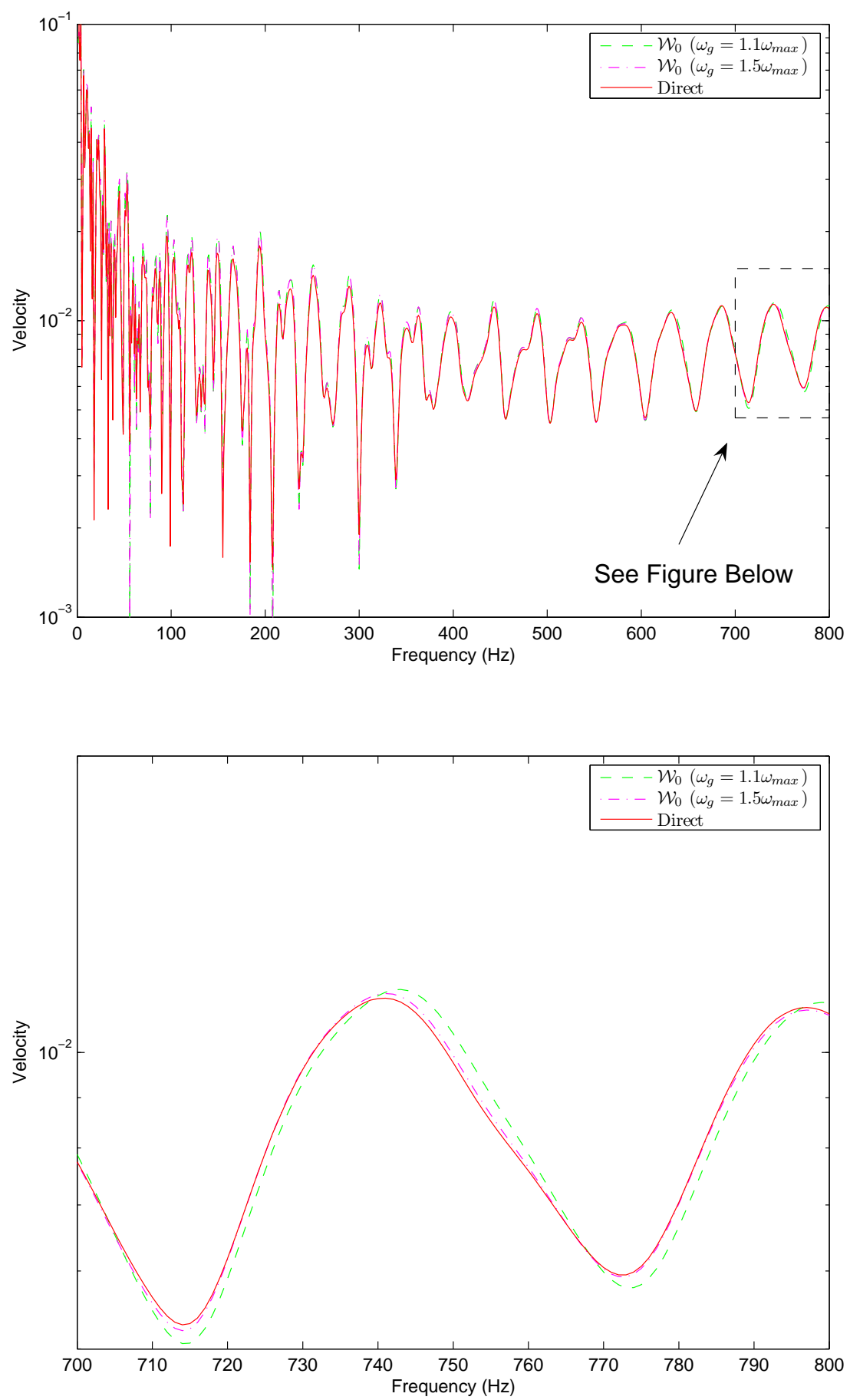

Figure 6.6: Effect of the global cutoff frequency $\omega_{g}$ on solution accuracy for the cantilever plate model with inset in bottom graph 
Table 6.1: Effect of the global cutoff frequency $\omega_{g}$ on elapsed time for all steps of the solution algorithm for the cantilever plate model

\begin{tabular}{|l||c|c|}
\hline \multirow{2}{*}{\multicolumn{1}{|c||}{ Step }} & \multicolumn{2}{c|}{ Approximate Solution } \\
\cline { 2 - 3 } & $\begin{array}{c}\mathcal{W}_{0}\left(\omega_{g}=1.1 \omega_{\max }\right) \\
(\mathrm{mm}: \mathrm{ss})\end{array}$ & $\begin{array}{c}\mathcal{W}_{0}\left(\omega_{g}=1.5 \omega_{\text {max }}\right) \\
(\mathrm{mm}: \mathrm{ss})\end{array}$ \\
\hline \hline Generate FE matrices & $03: 24$ & $03: 24$ \\
\hline Approximate eigensolution & $02: 24$ & $02: 35$ \\
\hline $\begin{array}{l}\text { Project system matrices onto } \\
\text { the approximating subspace }\end{array}$ & $00: 25$ & $00: 41$ \\
\hline $\begin{array}{l}\text { Compute solution on the } \\
\text { approximating subspace }\end{array}$ & $09: 02$ & $20: 16$ \\
\hline $\begin{array}{l}\text { Backtransform solution } \\
\text { to FE subspace }\end{array}$ & $00: 01$ & $00: 01$ \\
\hline \hline Total & $15: 16$ & $26: 57$ \\
\hline
\end{tabular}

Table 6.2: Effect of the global cutoff frequency $\omega_{g}$ for the cantilever plate model

\begin{tabular}{|l||c|c|}
\hline \multirow{2}{*}{\multicolumn{1}{|c||}{ Parameter }} & \multicolumn{2}{c|}{ Approximate Solution } \\
\cline { 2 - 3 } & $\mathcal{W}_{0}\left(\omega_{g}=1.1 \omega_{\max }\right)$ & $\mathcal{W}_{0}\left(\omega_{g}=1.5 \omega_{\max }\right)$ \\
\hline \hline Dimension of $\mathcal{A}$ subspace & 5782 & 6652 \\
\hline Dimension of $\mathcal{D}$ subspace & 3606 & 4325 \\
\hline Dimension of $\mathcal{E}$ subspace & 1052 & 1439 \\
\hline Number of RFVs & 1 & 1 \\
\hline Number of DDVs & 1 & 1441 \\
\hline $\begin{array}{l}\text { Dimension of } \\
\text { approximating subspace }\end{array}$ & 1054 & 1440 \\
\hline
\end{tabular}

Table 6.2). Table 6.1 shows that this $36.7 \%$ increase in the dimension of the approximating subspace results in an increase in the elapsed time to compute the approximate solution from 9 minutes and 2 seconds to 20 minutes and 16 seconds. This 11 minutes and 4 seconds increase in elapsed time is due to the fact that the leading term in the number of operations required for solutions computed on the $\mathcal{W}_{0}$ subspace is proportional to the dimension of the subspace cubed because the 
coefficient matrix is always fully populated due to the presence of frequency dependent materials in the FE model. Table 6.1 also shows that the time to compute the global eigenpairs and project system matrices onto the $\mathcal{W}_{0}$ subspace also increases because the AMLS, distilled, and refined subspaces are larger. Next, we will discuss how DRVs are computed for the 8 residuals whose energy seminorm ratio is above the user supplied cutoff.

\subsubsection{Effect of the Selection of Dynamic Response Vectors (DRVs)}

As explained in Chapter 5, DRVs are corrections to the approximate solutions of the reduced interpolated FRP, Eq. (4.10), at specific frequencies. The DRVs are computed on the $\mathcal{D}$ subspace at a significantly lower computational cost than computing DRVs on the $\mathcal{F}$ subspace. Also, DRVs computed on the $\mathcal{D}$ subspace are of excellent accuracy if the $\mathcal{D}$ subspace is constructed appropriately, with subtrees defined carefully and cutoff frequencies for subtree eigenvectors chosen well. In this section we will discuss the selection of frequencies at which the DRVs are computed.

Figure 6.7 shows the values of the energy seminorm ratio of the residual for 16 equally spaced DRV frequencies between 650 and $800 \mathrm{~Hz}$. It makes sense to choose DRV frequencies near $\omega_{\max }$ because generally the error from using a certain approximating subspace, especially a truncated eigenspace, increases as frequency increases. The horizontal dashed line in Fig. 6.7 represents the user supplied cutoff, and in this case, we set the cutoff to pass $50 \%$ of the energy seminorm ratios. The number of DRV frequencies and user supplied cutoff are chosen arbitrarily for this 


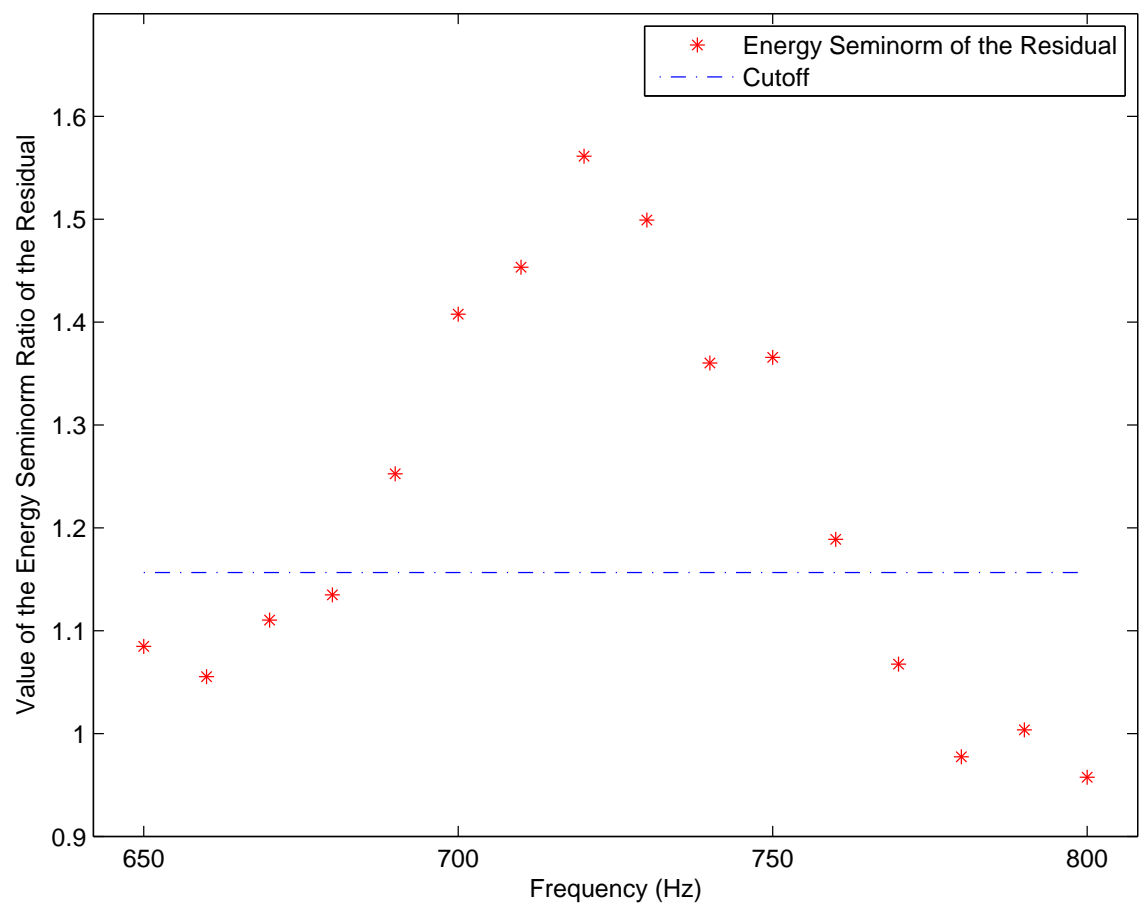

Figure 6.7: Values of the energy seminorm ratios of the residuals for the cantilever plate problem

FRP to demonstrate the general approach. In the next section, we will discuss how the 8 residuals, whose value of the energy seminorm ratio is above the user supplied cutoff, DRVs are computed.

\subsubsection{Effect of the Type of Dynamic Response Vectors (DRVs)}

DRVs can be accurately computed from Eq. (5.52) or inexpensively approximated from Eq. (5.53). Figure 6.8 shows that computing DRVs accurately from Eq. (5.52) does result in a more accurate approximate solution than inexpensively approximating DRVs from Eq. (5.53). However, Table 6.3 shows that accurately computing 
Table 6.3: Effect of including DRVs in the approximating subspace on elapsed time for all steps of the solution algorithm for the cantilever plate model

\begin{tabular}{|l||c|c|}
\hline \multicolumn{1}{|l||}{} & \multicolumn{2}{|c|}{ Approximate Solution } \\
\cline { 2 - 3 } Step & $\begin{array}{c}\mathcal{W} \text { (inexpensive) } \\
\text { (mm:ss) }\end{array}$ & $\begin{array}{c}\mathcal{W} \text { (accurate) } \\
\text { (mm:ss) }\end{array}$ \\
\hline \hline Generate FE matrices & $03: 24$ & $03: 24$ \\
\hline Approximate eigensolution & $02: 25$ & $02: 47$ \\
\hline Compute DRVs & $02: 12$ & $03: 24$ \\
\hline $\begin{array}{l}\text { Project system matrices } \\
\text { the approximating subspace }\end{array}$ & $00: 24$ & $00: 24$ \\
\hline $\begin{array}{l}\text { Compute solution on the } \\
\text { approximating subspace }\end{array}$ & $07: 31$ & $09: 29$ \\
\hline $\begin{array}{l}\text { Backtransform solution } \\
\text { to FE subspace }\end{array}$ & $00: 01$ & $00: 01$ \\
\hline \hline Total & $15: 57$ & $19: 29$ \\
\hline
\end{tabular}

DRVs from Eq. (5.52) takes $26.2 \%$ more elapsed time than inexpensively approximating the DRVs from Eq. (5.53). The effectiveness of inexpensively approximating DRVs on a more practical FRP is examined more thoroughly in Subsection 6.4.6.

Computing DRVs accurately from Eq. (5.52) is computationally intensive because of the high cost of factoring the large complex-valued coefficient matrix $A_{D}\left(\omega_{k}\right)$. To reduce the computational cost of factoring $A_{D}\left(\omega_{k}\right)$ in Eq. (5.52), a sequential version of the public domain sparse solver called MUltifrontal Massively Parallel sparse direct Solver (MUMPS) is used. Initially, we treated the $A_{D}\left(\omega_{k}\right)$ matrix as fully populated, but using MUMPS decreases the cost of factoring $A_{D}\left(\omega_{k}\right)$ by a factor of three by taking advantage of the known block sparsity pattern of $A_{D}\left(\omega_{k}\right)$. For the cantilever plate FRP, $34.9 \%$ of values in the coefficient matrix $A_{D}\left(\omega_{k}\right)$ are non-zero, and storing the fully populated coefficient matrix on the $\mathcal{D}$ 

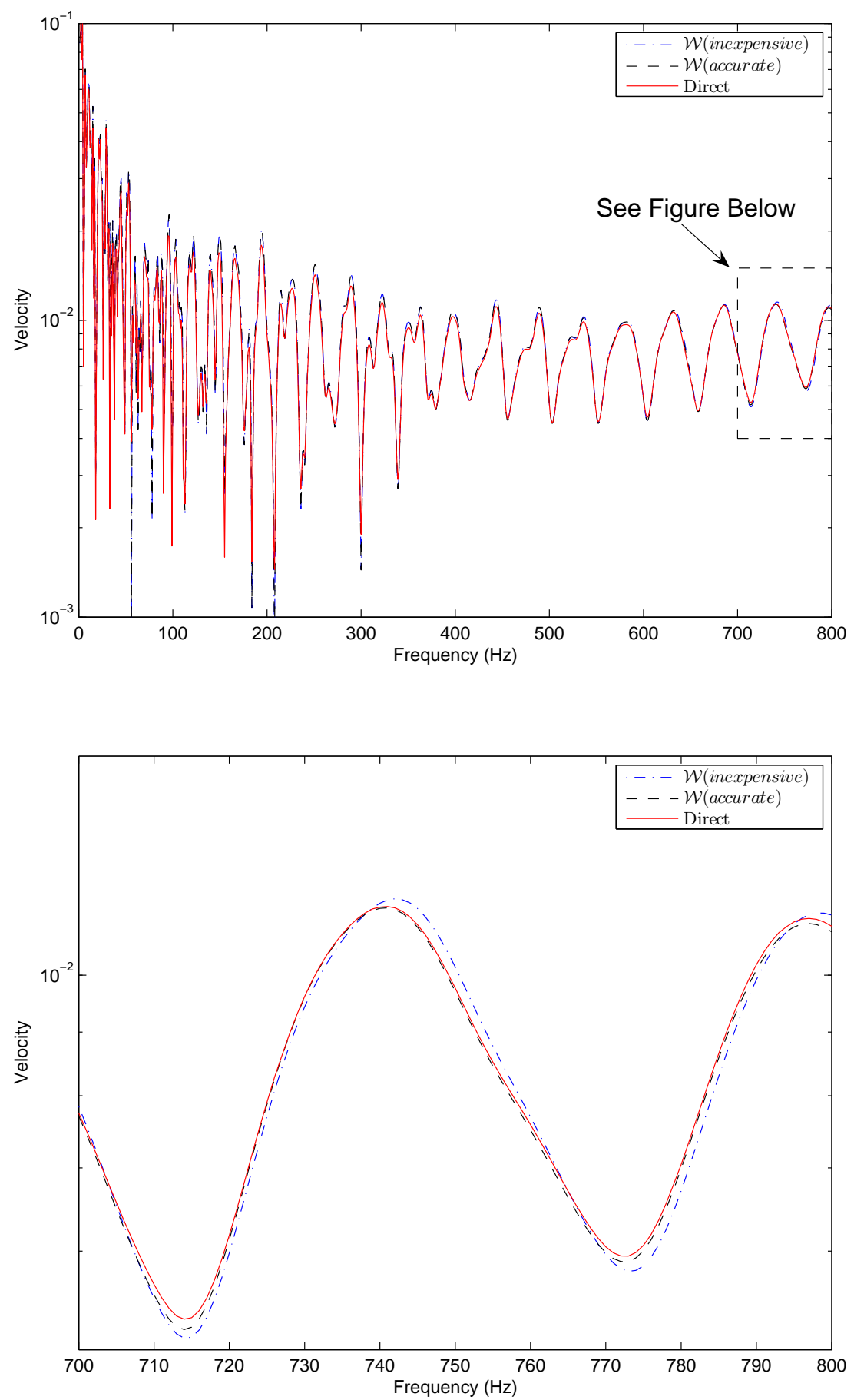

Figure 6.8: Effect of including DRVs in the approximating subspace on solution accuracy for the cantilever plate model with inset in bottom graph 
subspace takes a little over 200 Mbytes compared to a little over 50 Mbytes using a sparse format.

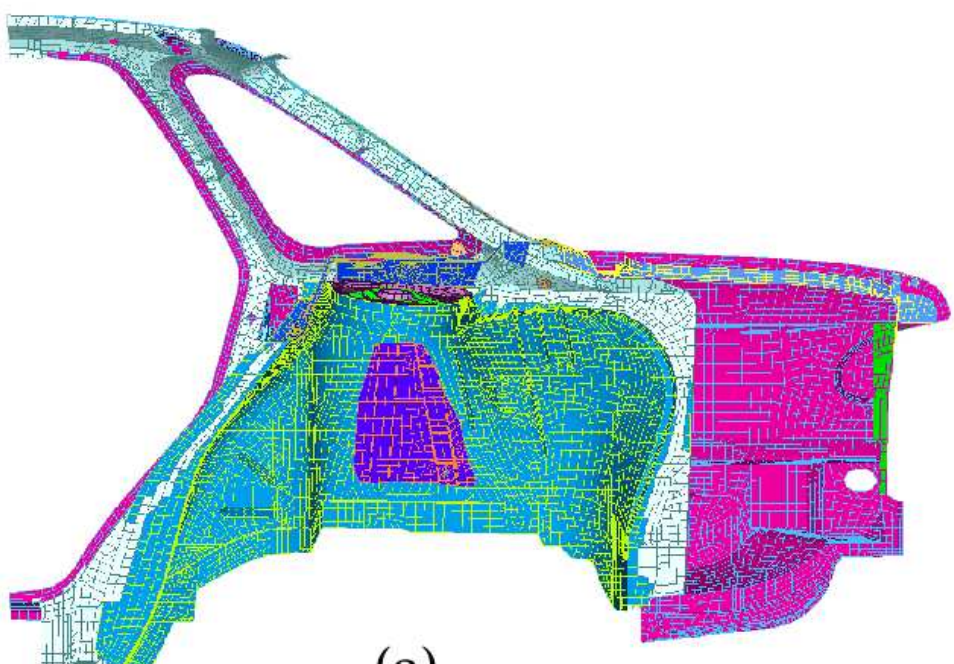

(a)

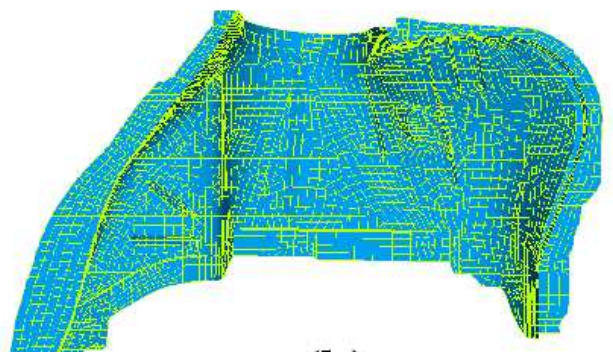

(b)

Figure 6.9: (a) FE representation of a automobile quarter panel (b) FE representation of the wheel house

\subsubsection{Quarter Panel FE Model}

Before the last parameter of Group Two is discussed, a practical application from the automotive industry is introduced. Figure 6.9(a) shows the FE representation of 
a 147,828 FE DOF quarter panel model that was developed by an automobile company. The shell elements that represent the wheel house shown in Fig. 6.9(b) were replaced with equivalent shell elements, as explained in Section 2.3, that represent a CLD configured sandwich of steel/VEM/steel. The input force is applied at the top of the shock tower, and the FE model includes a viscous damper and spring to model the shock absorber and suspension spring, respectively. Computing a direct solution of the interpolated FRP, Eq. (4.10), at 800 frequencies takes 13 hours and 46 minutes on a single microprocessor for the quarter panel FRP.

The last parameter of Group Two, the substructure cutoff frequency $\omega_{A}$, is discussed in the next section in relation to the quarter panel FE model. We could have used the cantilever plate model for this upcoming discussion, but solution accuracy is more sensitive to changes in the substructure cutoff frequency $\omega_{A}$ in the quarter panel model.

\subsubsection{Effect of the Substructure Cutoff Frequency}

As discussed in Chapter 5 , the substructure cutoff frequency $\omega_{A}$ determines the dimension of the $\mathcal{A}$ subspace, and the distillation cutoff frequency $\omega_{D}$ determines the dimension of the $\mathcal{D}$ subspace. The cutoff frequencies $\omega_{D}$ and $\omega_{A}$ can be adjusted independently; however, $\omega_{D}$ and $\omega_{A}$ are normally related to each other through the relationship $\omega_{D}=0.6 \omega_{A}$. For computing the eigensolution, setting $\omega_{D}$ to equal $0.6 \omega_{A}$ has been found by Kim [27] to yield accurate eigenpairs while minimizing elapsed time. We also studied the sensitivity of frequency response accuracy to the 
Table 6.4: Effect of substructure cutoff frequency $\omega_{A}$ on elapsed time for all steps of the solution algorithm for the quarter panel model

\begin{tabular}{|l||c|c|c|}
\hline \multicolumn{1}{|c||}{} & \multicolumn{3}{c|}{ Approximate Solution } \\
\cline { 2 - 4 } Step & $\begin{array}{c}\omega_{A}=5.0 \omega_{g} \\
(\mathrm{~mm}: \mathrm{ss})\end{array}$ & $\begin{array}{c}\omega_{A}=7.0 \omega_{g} \\
(\mathrm{~mm}: \mathrm{ss})\end{array}$ & $\begin{array}{c}\omega_{A}=9.0 \omega_{g} \\
(\mathrm{~mm}: \mathrm{ss})\end{array}$ \\
\hline \hline Generate FE matrices & $02: 51$ & $02: 51$ & $02: 51$ \\
\hline Approximate eigensolution & $06: 55$ & $07: 01$ & $07: 56$ \\
\hline Compute DRVs & $00: 42$ & $01: 34$ & $03: 03$ \\
\hline $\begin{array}{l}\text { Project system matrices onto } \\
\text { the approximating subspace }\end{array}$ & $00: 07$ & $00: 09$ & $00: 11$ \\
\hline $\begin{array}{l}\text { Compute solution on the } \\
\text { approximating subspace }\end{array}$ & $00: 35$ & $00: 36$ & $00: 37$ \\
\hline $\begin{array}{l}\text { Backtransform solution } \\
\text { to FE subspace }\end{array}$ & $00: 03$ & $00: 03$ & $00: 03$ \\
\hline \hline Total & $11: 13$ & $12: 14$ & $14: 41$ \\
\hline
\end{tabular}

choice of $\omega_{D}$ and found that the accuracy is much more sensitive to $\omega_{A}$ than $\omega_{D}$, and setting $\omega_{D}$ equal to $0.6 \omega_{A}$ always resulted in nearly all of the accuracy available from a given choice of $\omega_{A}$ being achieved.

Choosing $\omega_{A}$ equal to a default value of $5.0 \omega_{g}$ is a good starting point because this default value for $\omega_{A}$ was determined heuristically for computing accurate eigenpairs on numerous FRPs. For the cantilever plate FRP, accurate approximate solutions are obtained using the default value $\omega_{A}=5.0 \omega_{g}$. However, for the quarter panel and the soon to be discussed "body-in-white" models, the accuracy of the DRVs must be improved to obtain more accurate approximate solutions by increasing $\omega_{A}$ while maintaining $\omega_{g}$ at $1.1 \omega_{\max }$.

Figure 6.10 illustrates the effect of the substructure cutoff frequency $\omega_{A}$ on solution accuracy for the quarter panel model. As expected, approximate solution 

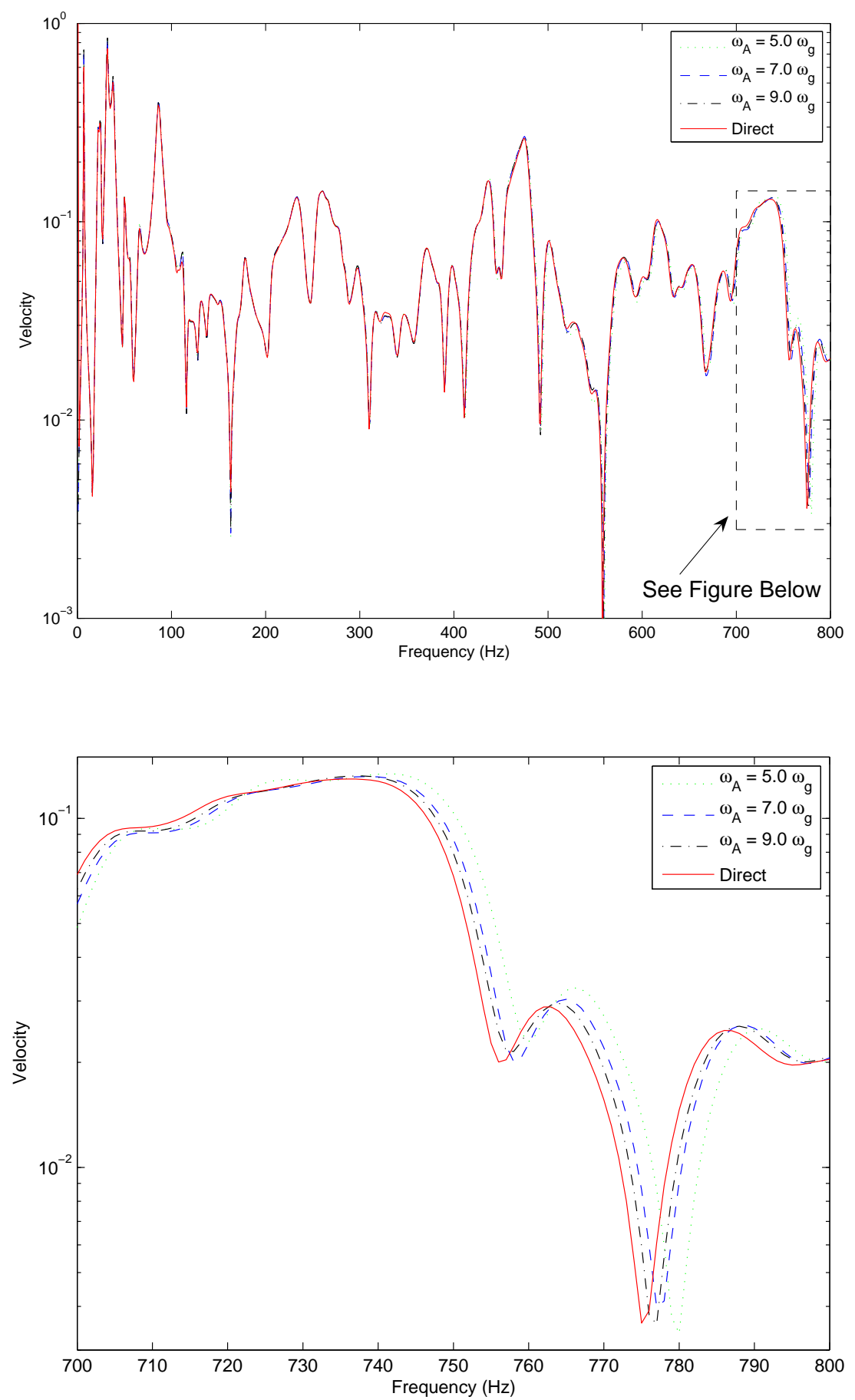

Figure 6.10: Effect of substructure cutoff frequency $\omega_{A}$ on solution accuracy for the quarter panel model with inset in bottom graph 
Table 6.5: Effect of substructure cutoff frequency $\omega_{A}$ for the quarter panel model

\begin{tabular}{|l||c|c|c|}
\hline \multirow{2}{*}{\multicolumn{1}{|c||}{ Parameters }} & \multicolumn{3}{c|}{ Approximate Solution } \\
\cline { 2 - 4 } & $\omega_{A}=5.0 \omega_{g}$ & $\omega_{A}=7.0 \omega_{g}$ & $\omega_{A}=9.0 \omega_{g}$ \\
\hline \hline Substructure cutoff frequency $\omega_{A}$ & $5.0 \omega_{g}$ & $7.0 \omega_{g}$ & $9.0 \omega_{g}$ \\
\hline Nominal frequency $\omega_{D}$ & $3.0 \omega_{g}$ & $4.2 \omega_{g}$ & $5.4 \omega_{g}$ \\
\hline Dimension of $\mathcal{A}$ subspace & 4709 & 6894 & 9362 \\
\hline Dimension of $\mathcal{D}$ subspace & 1900 & 2968 & 4300 \\
\hline Dimension of $\mathcal{E}$ subspace & 345 & 348 & 348 \\
\hline Number of RFVs & 1 & 1 & 1 \\
\hline Number of DRVs & 14 & 14 & 14 \\
\hline $\begin{array}{l}\text { Dimension of } \\
\text { approximating subspace }\end{array}$ & 360 & 363 & 363 \\
\hline
\end{tabular}

accuracy increases for larger values of $\omega_{A}$ because the DRVs are computed on larger $\mathcal{D}$ subspaces (see Table 6.5). Approximate solution accuracy increases substantially by increasing $\omega_{A}$ from $5.0 \omega_{g}$ to $7.0 \omega_{g}$, but the increase in solution accuracy is not as significant when increasing $\omega_{A}$ from $7.0 \omega_{g}$ to $9.0 \omega_{g}$. The total elapsed time only increases by $9.1 \%$ when increasing $\omega_{A}$ from $5.0 \omega_{g}$ to $7.0 \omega_{g}$ compared to a $30.1 \%$ increase when increasing $\omega_{A}$ from $5.0 \omega_{g}$ to $9.0 \omega_{g}$ (see Table 6.4). A balance between DRV accuracy and total elapsed time is achieved by setting $\omega_{A}$ to $7.0 \omega_{g}$.

\subsubsection{New Approach Applied to Quarter Panel Model}

Before introducing the last FRP, some valuable insights can be gained by looking at the cost of computing DRVs. Figure 6.11 compares approximate solutions computed on four different approximating subspaces. The increase in accuracy by including inexpensively approximated DRVs from Eq. (5.53) is almost negligible, which indicates that accurately computing the DRVs is important to the accuracy of the solution. 

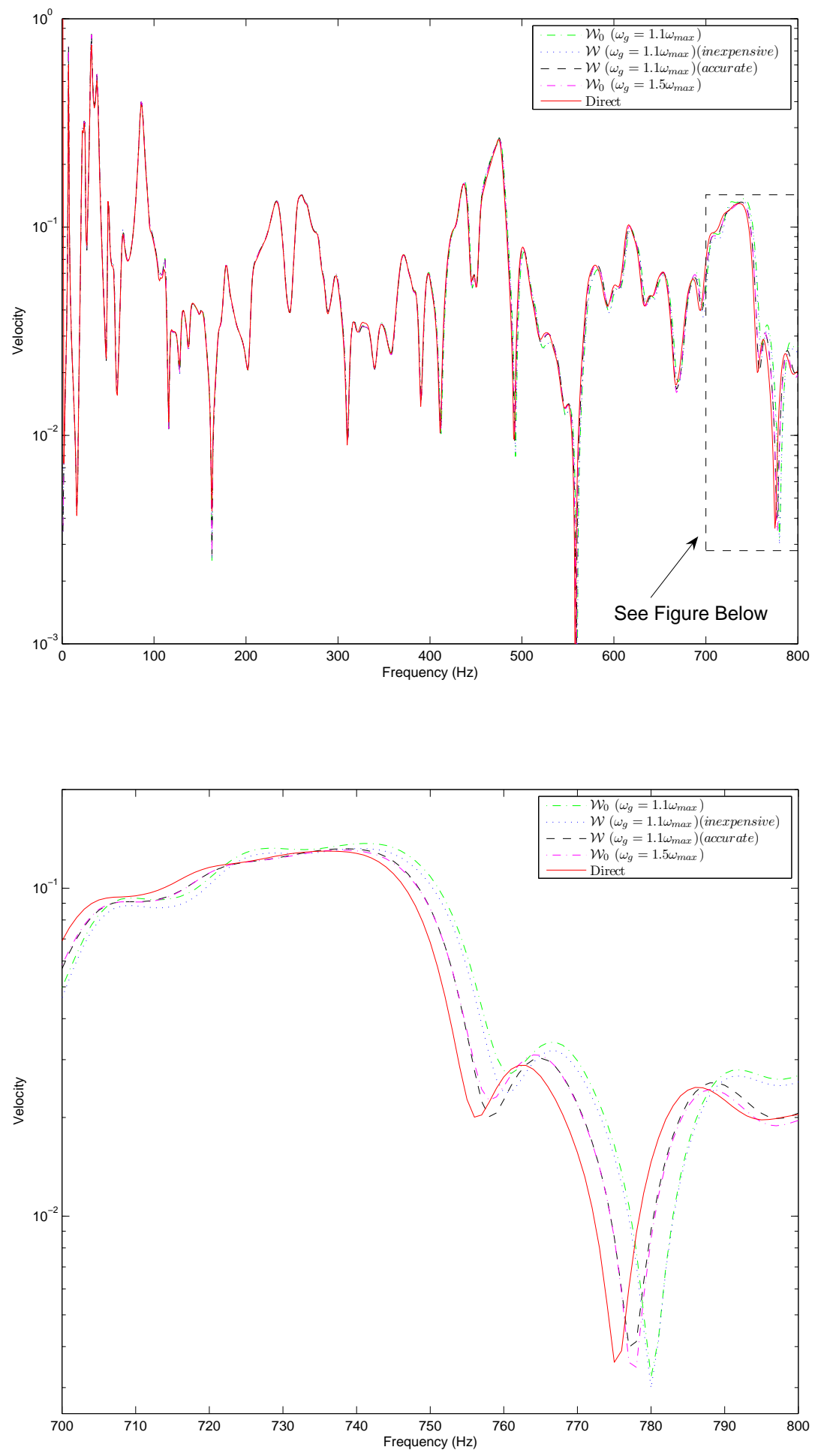

Figure 6.11: Comparison of four approximate solutions for the quarter panel model with inset in bottom graph 
Table 6.6: Comparison of the elapsed time for all steps of the solution algorithm for the quarter panel model

\begin{tabular}{|l||c|c|}
\hline \multicolumn{1}{|l||}{} & \multicolumn{2}{c|}{ Approximate Solution } \\
\cline { 2 - 3 } Step & $\begin{array}{c}\mathcal{W}_{0} \\
\omega_{g} 1.1 \omega_{\max } \\
(\mathrm{mm}: \mathrm{ss})\end{array}$ & $\begin{array}{c}\mathcal{W} \text { (inexpensive) } \\
\omega_{g}=1.1 \omega_{\max } \\
(\mathrm{mm}: \mathrm{ss})\end{array}$ \\
\hline \hline Generate FE matrices & $02: 51$ & $02: 51$ \\
\hline Approximate eigensolution & $07: 01$ & $07: 01$ \\
\hline Compute DRV & $00: 00$ & $00: 52$ \\
\hline $\begin{array}{l}\text { Project system matrices onto } \\
\text { the approximating subspace }\end{array}$ & $00: 09$ & $00: 09$ \\
\hline $\begin{array}{l}\text { Compute solution on the } \\
\text { approximating subspace }\end{array}$ & $00: 37$ & $00: 36$ \\
\hline Backtransform solution to FE subspace & $00: 03$ & $00: 03$ \\
\hline \hline Total & $10: 41$ & $11: 33$ \\
\hline
\end{tabular}

Table 6.7: Comparison of the elapsed time for all steps of the solution algorithm for the quarter panel model

\begin{tabular}{|l||c|c|}
\hline \multicolumn{1}{|l||}{} & \multicolumn{2}{c|}{ Approximate Solution } \\
\cline { 2 - 3 } \multicolumn{1}{|l|}{ Step } & $\begin{array}{c}\mathcal{W} \text { (accurate) } \\
\omega_{g}=1.1 \omega_{\max } \\
(\mathrm{mm}: \mathrm{ss})\end{array}$ & $\begin{array}{c}\mathcal{W}_{0} \\
\omega_{g}=1.5 \omega_{\max } \\
(\mathrm{mm}: \mathrm{ss})\end{array}$ \\
\hline \hline Generate FE matrices & $02: 51$ & $02: 51$ \\
\hline Approximate eigensolution & $07: 01$ & $07: 26$ \\
\hline Compute DRV & $01: 34$ & $00: 00$ \\
\hline $\begin{array}{l}\text { Project system matrices onto } \\
\text { the approximating subspace }\end{array}$ & $00: 09$ & $00: 14$ \\
\hline $\begin{array}{l}\text { Compute solution on the } \\
\text { approximating subspace }\end{array}$ & $00: 36$ & $01: 49$ \\
\hline Backtransform solution to FE subspace & $00: 03$ & $00: 03$ \\
\hline \hline Total & $12: 14$ & $12: 23$ \\
\hline
\end{tabular}

Other FRPs that have complex FE model geometry and are modally dense also exhibited the same negligible benefit of including inexpensively approximated DRVs in the approximating subspace. In the next chapter, future work is suggested that 
Table 6.8: Parameters of the solution approach for the quarter panel model

\begin{tabular}{|l||c|c|c|}
\hline \multicolumn{1}{|l||}{} & \multicolumn{3}{c|}{ Approximate Solution } \\
\cline { 2 - 4 } Parameter & $\begin{array}{c}\mathcal{W}_{0} \\
\omega_{g}=1.1 \omega_{\max }\end{array}$ & $\begin{array}{c}\mathcal{W} \\
\omega_{g}=1.1 \omega_{\max }\end{array}$ & $\begin{array}{c}\mathcal{W}_{0} \\
\omega_{g}=1.5 \omega_{\text {max }}\end{array}$ \\
\hline \hline $\begin{array}{l}\text { Substructure cutoff } \\
\text { frequency } \omega_{A}\end{array}$ & $7.0 \omega_{g}$ & $7.0 \omega_{g}$ & $5.0 \omega_{g}$ \\
\hline $\begin{array}{l}\text { Distillation cutoff } \\
\text { frequency } \omega_{D}\end{array}$ & $4.2 \omega_{g}$ & $4.2 \omega_{g}$ & $3.0 \omega_{g}$ \\
\hline Dimension of $\mathcal{A}$ subspace & 6894 & 6894 & 6808 \\
\hline Dimension of $\mathcal{D}$ subspace & 2968 & 2968 & 2920 \\
\hline Dimension of $\mathcal{E}$ subspace & 348 & 348 & 567 \\
\hline Number of RFVs & 1 & 1 & 1 \\
\hline Number of DDVs & 0 & 0 & 0 \\
\hline Number of DRVs & 0 & 16 & 0 \\
\hline $\begin{array}{l}\text { Dimension of } \\
\text { approximating subspace }\end{array}$ & 349 & 365 & 568 \\
\hline
\end{tabular}

includes evaluating iterative methods to compute DRVs as inexpensively as possible while attaining a high level of DRV accuracy.

Figure 6.11 also shows that approximate solutions of almost identical accuracy can be computed by either increasing $\omega_{g}$ or including DRVs in the approximating subspace. Therefore, we were able to achieve the same level of accuracy by replacing 215 eigenvectors with 16 DRVs. However, Table 6.7 shows that the approximate solution computed when $\omega_{g}=1.5 \omega_{\max }$ only takes 9 seconds longer. The time it takes to compute the DRVs almost outweighs the time saved solving the interpolated reduced FRP, Eq. (5.60), on a smaller approximating subspace. Therefore, the applicability of the solution approach to FRPs looks limited to modally dense FRPs, unless the cost of accurately computing DRVs or the number of DRVs can be reduced. For this example problem, the dimension of the eigenspace is in 
the hundreds, while the industrial applications motivating this research typically have thousands of modes participating in the FRP so that the FRP is much more expensive to solve. For this reason, a FRP that is much more modally dense is solved in the next section.

\section{5 "Body-in-White" Automobile FE Model}

Figure 6.12(a) shows the FE representation of a "body-in-white" automobile body structural assembly. This model has 232,296 FE DOF and is included in this chapter to demonstrate the applicability of the solution approach to a representative FRP found in the automotive industry. The number of FE DOF for this "Body-in-White" FRP is less than the several million FE DOF found in industrial FRPs, but the number of modes is getting closer to the current level of several thousand modes.

The dashboard, wheel houses, firewall, floor pan, and trunk well, shown in Fig. 6.12(b), are modified to include CLD configured VEMs. The "body-inwhite" FE model has four viscous dampers and four springs to model the shock absorbers and suspension springs, respectively. The input force is applied at the top of the driver's side front shock tower and the output measurement location is at the tip of the frame for the steering column. Computing the direct solution of the interpolated FRP, Eq. (4.10), at 800 frequencies takes 21 hours and 43 minutes on a single processor.

Figure 6.13 shows that the solution algorithm presented in this dissertation 


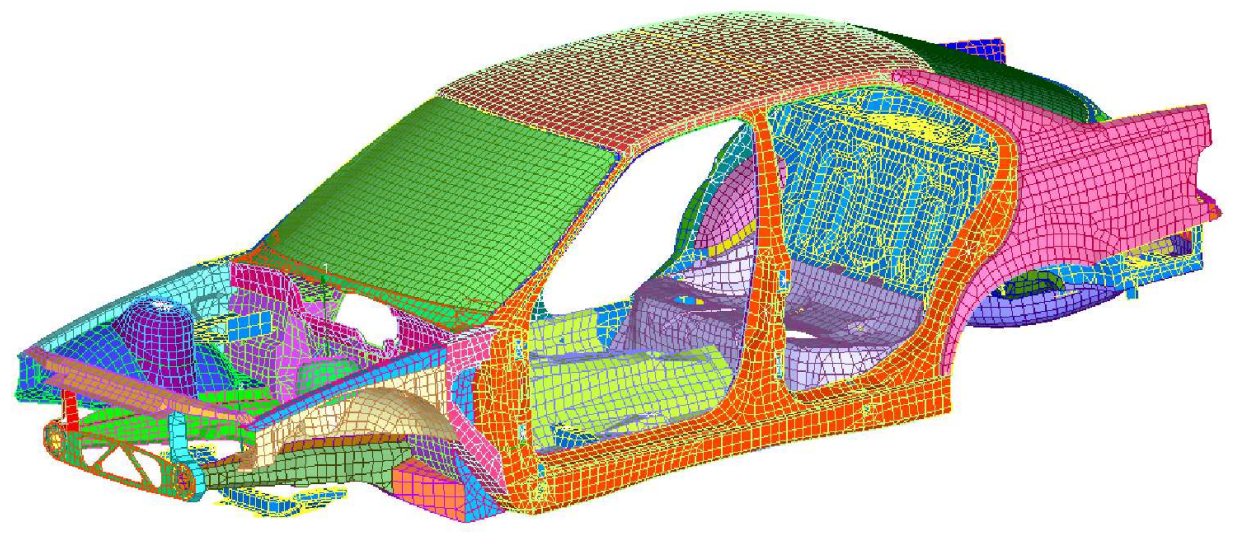

(a)
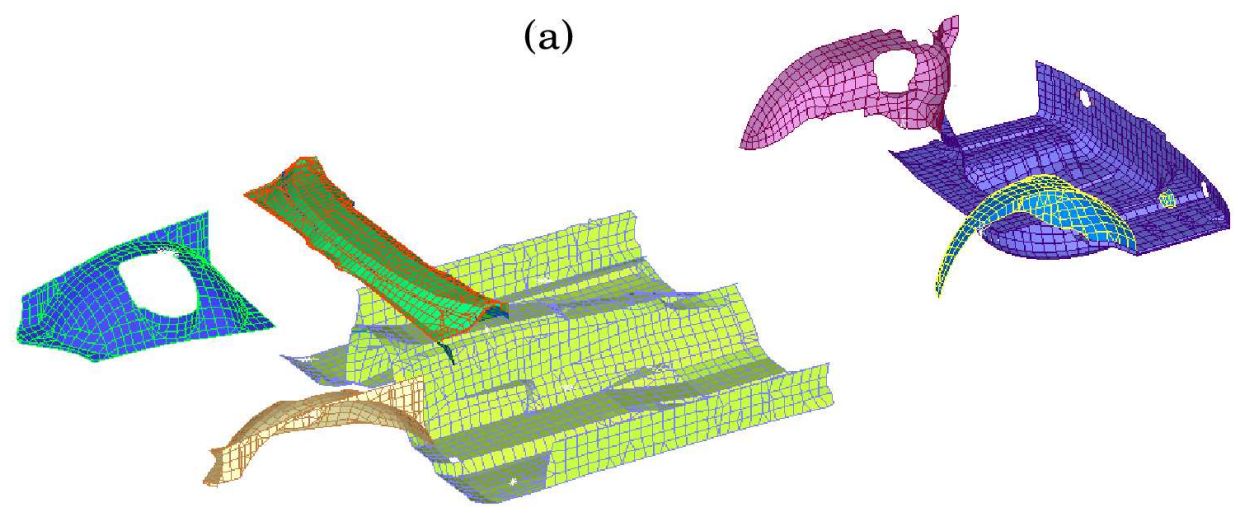

(b)

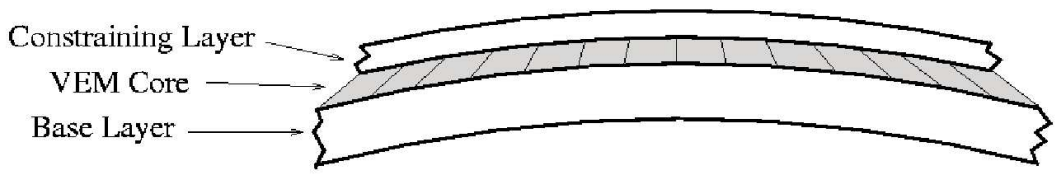

(c)

Figure 6.12: (a) FE representation of a "body-in-white" automobile body (b) FE representation of the dashboard, wheel houses, firewall, floor pan, and trunk well (c) Cross section of constrained layer damping sandwich 

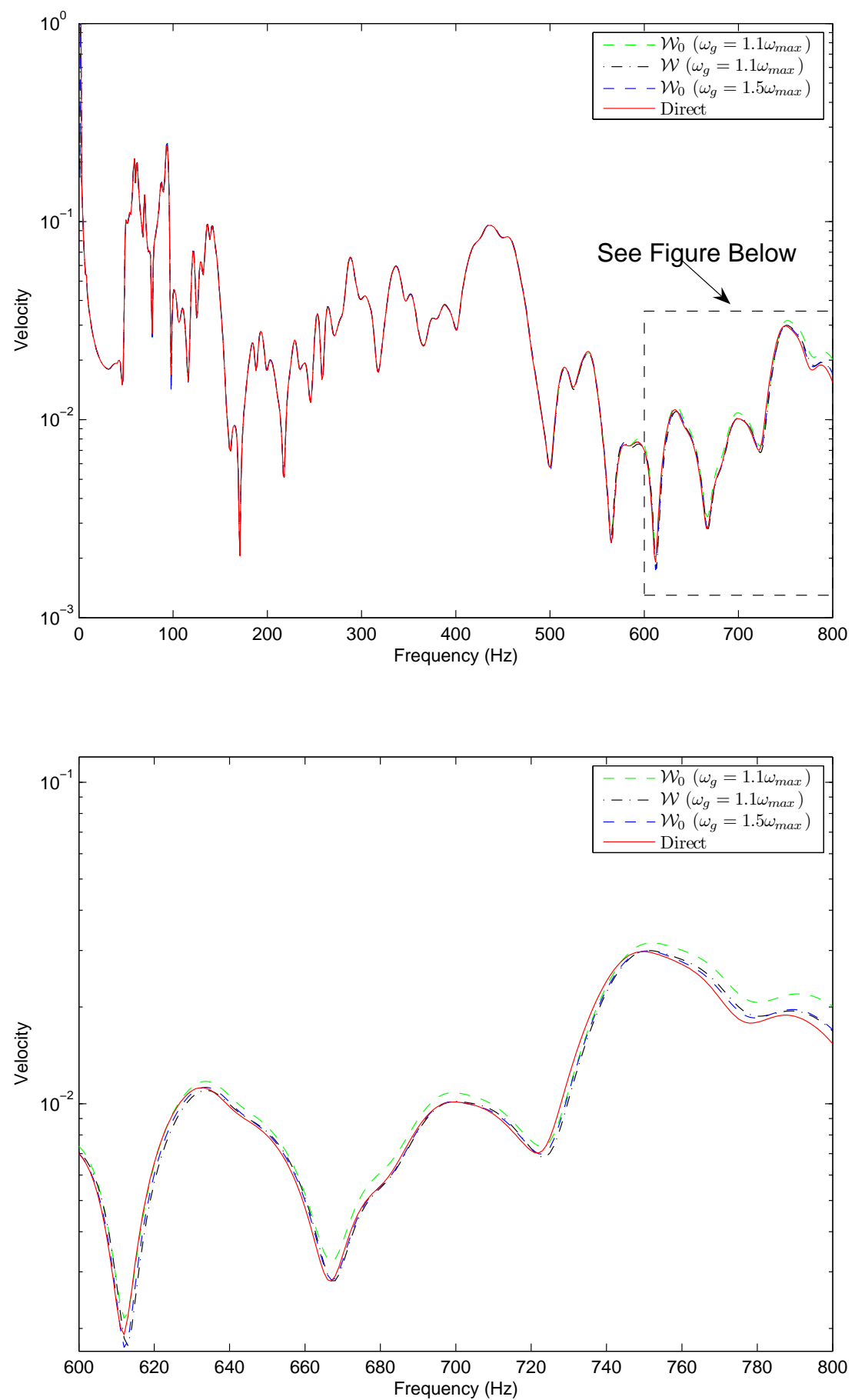

Figure 6.13: Accuracy of approximate solutions for the "body-in-white" model with inset in bottom graph 
Table 6.9: Parameters of the solution approach for the "body-in-white" model

\begin{tabular}{|l||c|c|}
\hline \multicolumn{1}{|c||}{} & \multicolumn{2}{c|}{ Approximate Solution } \\
\cline { 2 - 3 } Parameters & $\begin{array}{c}\mathcal{W} \\
\omega_{g}=1.1 \omega_{\max }\end{array}$ & $\begin{array}{c}\mathcal{W}_{0} \\
\omega_{g}\end{array} 1.5 \omega_{\max }$ \\
\hline \hline Substructure cutoff frequency $\omega_{A}$ & $7.0 \omega_{\max }$ & $5.0 \omega_{\max }$ \\
\hline Dimension of $\mathcal{A}$ subspace & 22782 & 22467 \\
\hline Dimension of $\mathcal{D}$ subspace & 13903 & 13689 \\
\hline Dimension of $\mathcal{E}$ subspace & 3334 & 5008 \\
\hline Number of RFVs & 1 & 1 \\
\hline Number of DDVs & 3 & 3 \\
\hline Number of DRVs & 16 & 0 \\
\hline $\begin{array}{l}\text { Dimension of } \\
\text { approximating subspace }\end{array}$ & 3354 & 5012 \\
\hline
\end{tabular}

Table 6.10: Comparison of the elapsed time for all steps of the solution algorithm for the "body-in-white" model

\begin{tabular}{|l||c|c|}
\hline \multicolumn{1}{|c||}{} & \multicolumn{2}{c|}{ Approximate Solution } \\
\cline { 2 - 3 } \multicolumn{1}{|l|}{ Step } & $\begin{array}{c}\mathcal{W} \\
\omega_{g}=1.1 \omega_{\max } \\
(\mathrm{hh}: \mathrm{mm}: \mathrm{ss})\end{array}$ & $\begin{array}{c}\mathcal{W}_{0} \\
\omega_{g}=1.5 \omega_{\text {max }} \\
\text { (hh:mm:ss) }\end{array}$ \\
\hline \hline Generate FE matrices & $00: 06: 06$ & $00: 06: 09$ \\
\hline Approximate eigensolution & $00: 13: 41$ & $00: 18: 05$ \\
\hline Compute DRVs & $02: 00: 15$ & $00: 00: 00$ \\
\hline $\begin{array}{l}\text { Project system matrices onto } \\
\text { the approximating subspace }\end{array}$ & $00: 09: 17$ & $00: 16: 02$ \\
\hline $\begin{array}{l}\text { Compute solution on the } \\
\text { approximating subspace }\end{array}$ & $03: 28: 38$ & $10: 54: 18$ \\
\hline Backtransform solution to FE subspace & $00: 00: 05$ & $00: 00: 05$ \\
\hline \hline Total & $05: 58: 02$ & $11: 34: 39$ \\
\hline
\end{tabular}

can achieve similar accuracy as obtained by raising the cutoff frequency for global eigenpairs. Table 6.10 shows that it takes only 5 hours and 58 minutes to compute the approximate solution on the $\mathcal{W}$ subspace with $\omega_{g}=1.1 \omega_{\max }$ compared to the 11 hours and 34 minutes it takes to compute an approximate solution on the $\mathcal{W}_{0}$ 
Table 6.11: Effect of the substructure cutoff frequency $\omega_{A}$ on solution parameters for the "body-in-white" model

\begin{tabular}{|l||c|c|c|}
\hline \multicolumn{1}{|c||}{} & \multicolumn{3}{c|}{ Approximate Solution } \\
\cline { 2 - 4 } & $\begin{array}{c}\mathcal{W} \\
\omega_{A}=5.0 \omega_{g}\end{array}$ & $\begin{array}{c}\mathcal{W} \\
\omega_{A}=7.0 \omega_{g}\end{array}$ & $\begin{array}{c}\mathcal{W} \\
\omega_{A}=9.0 \omega_{g}\end{array}$ \\
\hline \hline Dimension of $\mathcal{A}$ subspace & 18762 & 22782 & 26019 \\
\hline Dimension of $\mathcal{D}$ subspace & 11029 & 13903 & 16101 \\
\hline Dimension of $\mathcal{E}$ subspace & 3325 & 3334 & 3337 \\
\hline Number of RFVs & 1 & 1 & 1 \\
\hline Number of DDVs & 3 & 3 & 3 \\
\hline Number of DRVs & 16 & 16 & 16 \\
\hline $\begin{array}{l}\text { Dimension of } \\
\text { approximating subspace }\end{array}$ & 3345 & 3354 & 3357 \\
\hline
\end{tabular}

subspace with $\omega_{g}=1.5 \omega_{\max }$. The difference in elapsed time is primarily due to the fact that the approximating subspace is $49 \%$ larger (see Table 6.9). In the end, we were able to replace 1,674 eigenvectors with 16 DRVs and achieve the same accuracy in less time. Next, we take another look at the effect of substructure cutoff frequency $\omega_{A}$ on solution accuracy and computational cost.

Previously, we set the substructure cutoff frequency $\omega_{A}$ equal to $7.0 \omega_{g}$ as a compromise between solution accuracy and elapsed time. Table 6.12 compares elapsed time for all the steps in computing DRVs for $\omega_{A}$ equal to $5.0 \omega_{g}, 7.0 \omega_{g}$, and $9.0 \omega_{g}$. As expected, Figure 6.14 shows that solution accuracy can be increased by setting $\omega_{A}$ equal to $9.0 \omega_{g}$. However, the elapsed time for computing DRVs increases $52 \%$ compared to setting $\omega_{A}$ equal to $7.0 \omega_{g}$ (see Table 6.12 ). Figure 6.15 shows that increasing $\omega_{A}$ results in more accurate solutions than increasing the global cutoff frequency and the total elapsed time is still 4 hours and 33 minutes less. 

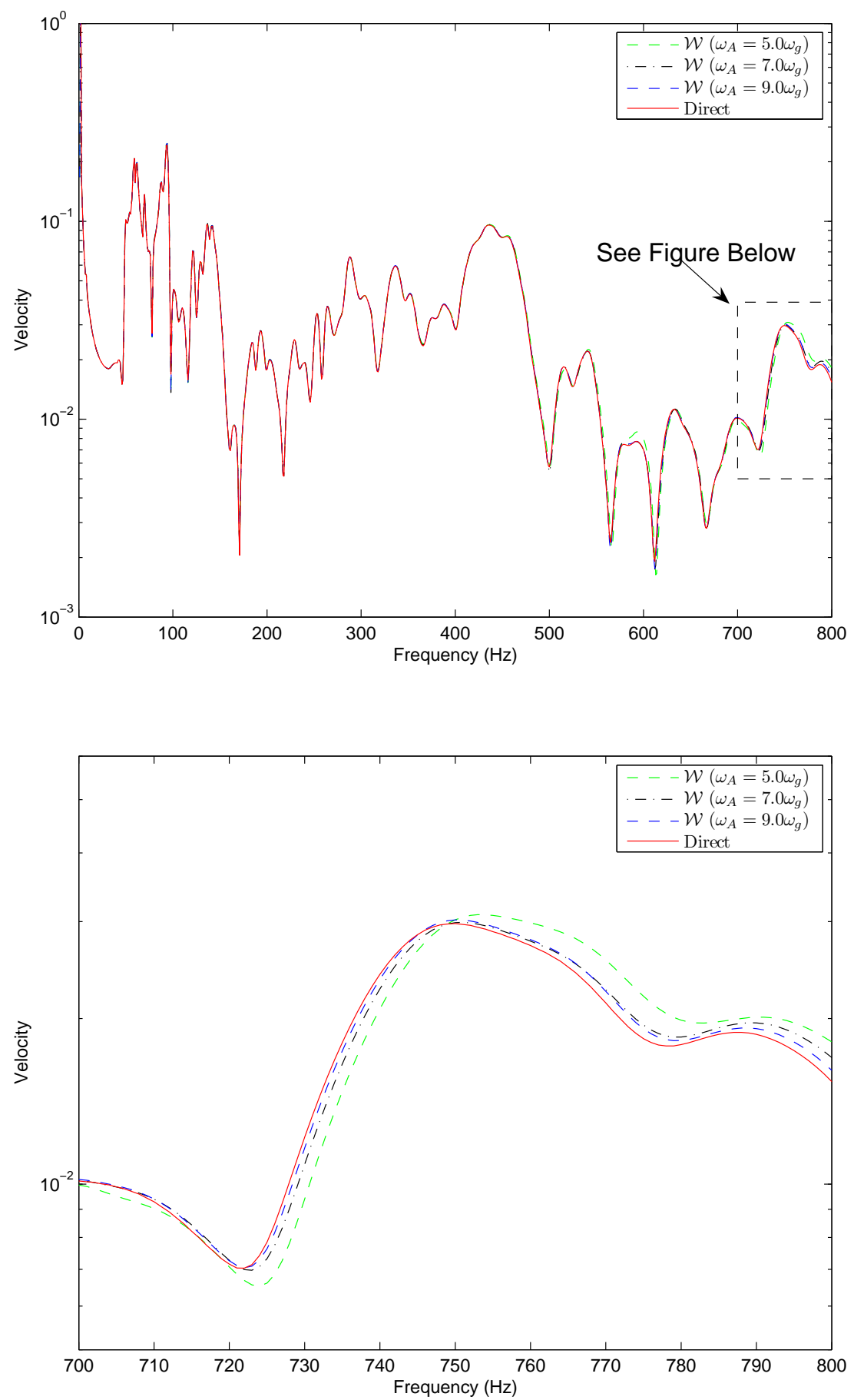

Figure 6.14: Effect of the substructure cutoff frequency $\omega_{A}$ on solution accuracy for the "body-in-white" model with inset in bottom graph 

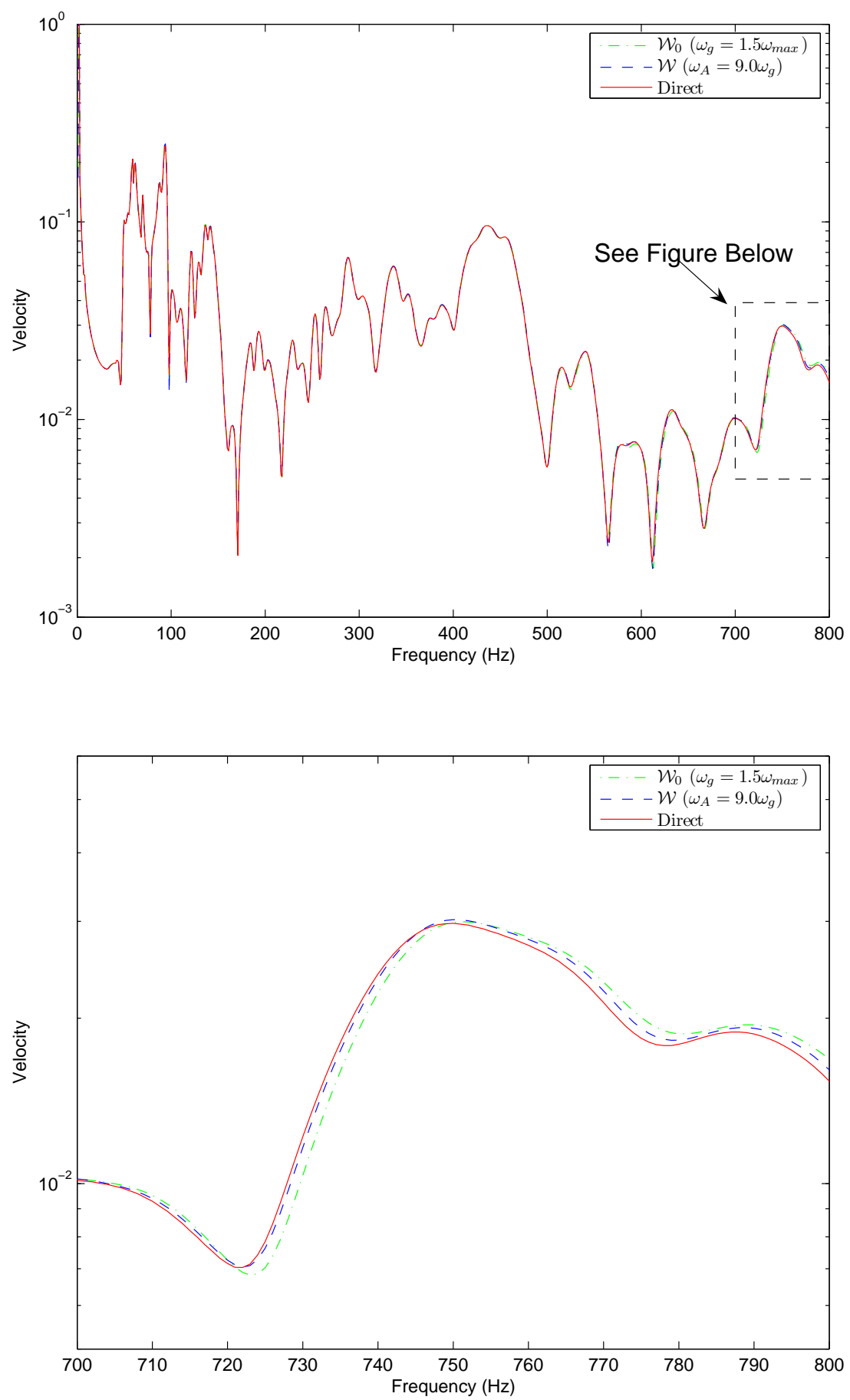

Figure 6.15: Comparison of approximate solutions for the "body-in-white" model with inset in bottom graph 
Table 6.12: Effect of the substructure cutoff frequency $\omega_{A}$ on elapsed time for the "body-in-white" model

\begin{tabular}{|l||c|c|c|}
\hline \multicolumn{1}{|c||}{} & \multicolumn{3}{c|}{ Approximate Solution } \\
\cline { 2 - 4 } Step & $\begin{array}{c}\mathcal{W} \\
\omega_{A}=5.0 \omega_{g} \\
(\mathrm{~mm}: \mathrm{ss})\end{array}$ & $\begin{array}{c}\mathcal{W} \\
\omega_{g}=7.0 \omega_{g} \\
(\mathrm{~mm}: \mathrm{ss})\end{array}$ & $\begin{array}{c}\mathcal{W} \\
\omega_{g}=9.0 \omega_{g} \\
(\mathrm{~mm}: \mathrm{ss})\end{array}$ \\
\hline $\begin{array}{l}\text { Transform } K_{j} \text { matrices } \\
\text { from the } \mathcal{A} \text { to } \mathcal{D} \text { subspace }\end{array}$ & $14: 07$ & $28: 57$ & $43: 17$ \\
\hline $\begin{array}{l}\text { Transform } K_{j} \text { matrices } \\
\text { from the } \mathcal{D} \text { to } \mathcal{W}_{0} \text { subspace }\end{array}$ & $14: 20$ & $20: 17$ & $25: 54$ \\
\hline Compute 16 solutions on $\mathcal{W}_{0}$ subspace & $02: 07$ & $02: 09$ & $02: 08$ \\
\hline Compute 8 DRVs on $\mathcal{D}$ subspace & $37: 06$ & $68: 52$ & $112: 18$ \\
\hline Backtransform DRVs to $\mathcal{A}$ subspace & $00: 03$ & $00: 06$ & $00: 08$ \\
\hline \hline Total & $77: 43$ & $120: 15$ & $183: 45$ \\
\hline
\end{tabular}

We can gain more insight into the solution approach by looking at the effect of the number of DRVs. Figure 6.16 shows that increasing the number of DRVs over 16 does not improve solution accuracy noticeably. Reducing the number of DRVs to 8 decreases solution accuracy. A near-optimal number of DRVs is expected to be between 8 and 16. It is interesting to realize that the cost of computing 8,16 , 24, and 32 initial approximations on the $\mathcal{W}_{0}$ subspace and the respective energy seminorm of their residuals is only $1.6 \%, 1.8 \%, 2.3 \%$, and $2.2 \%$ of the total elapsed time for Phase $4 \mathrm{~d}$, respectively. The average cost of transforming the $K_{j}$ matrices from the $\mathcal{D}$ to the $\mathcal{W}_{0}$ subspace is about $25 \%$ of the total elapsed time for Phase $4 \mathrm{~d}$. Simply stated, once we pay the cost of projecting the FRP into the $\mathcal{W}_{0}$ subspace, the cost computing initial approximations is so low that we can inexpensively determine the best frequencies at which to compute DRVs. Therefore, a more thorough search for the best frequencies to compute DRVs could reduce the total cost of Phase 
$4 \mathrm{~d}$ because computing DRVs accurately is the most expensive step in Phase $4 \mathrm{~d}$. This insight could lead to an adaptive approach where we would iteratively sample the accuracy of the approximate solution at different frequencies, compute DRVs at those frequencies where the approximate solution is least accurate, and include those DRVs in the approximate solution until a user defined cutoff is met. 

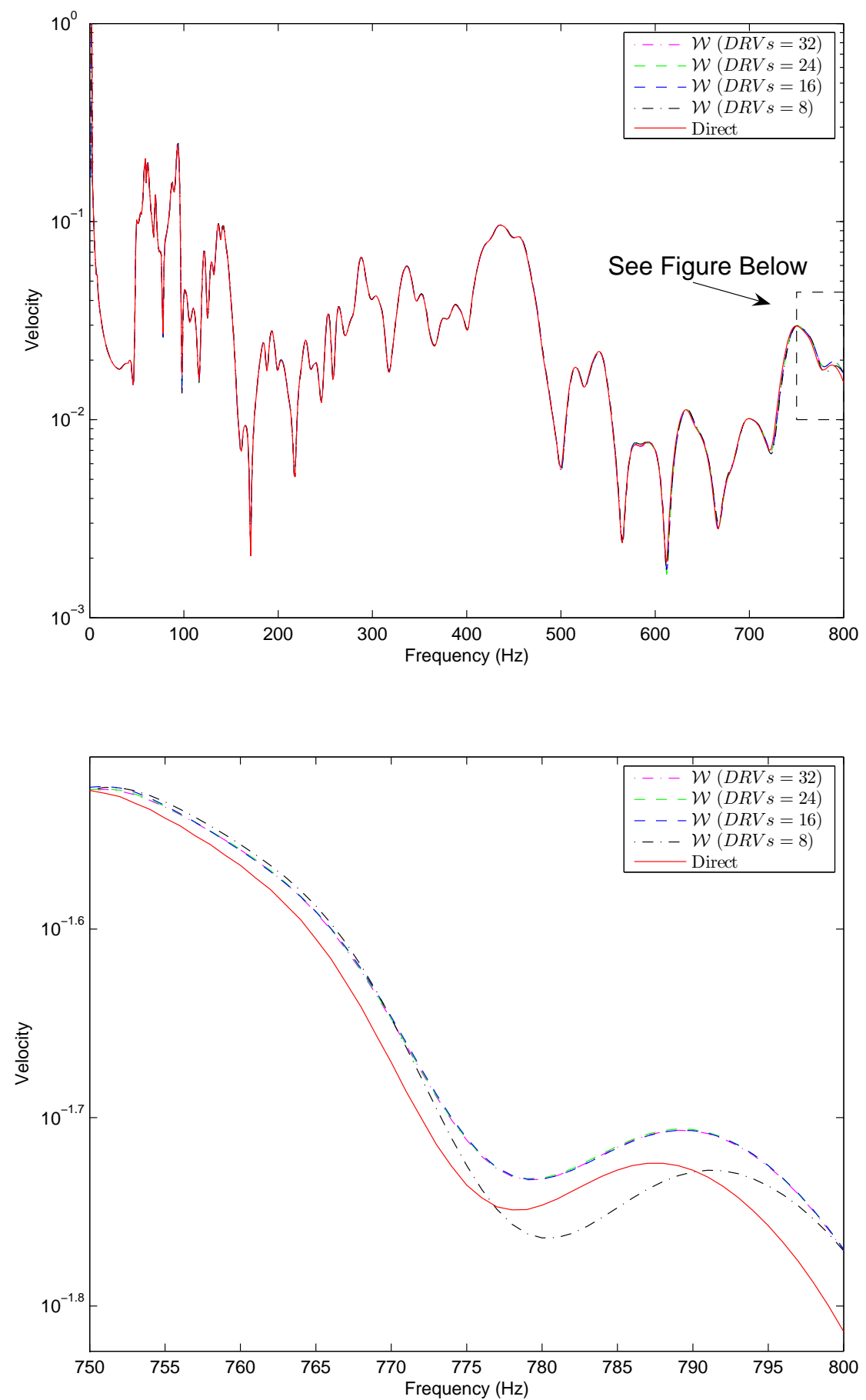

Figure 6.16: Effect of the number of DRVs on solution accuracy for the "body-inwhite" model with inset in bottom graph 


\section{Chapter 7}

\section{Conclusions and Future Work}

In this dissertation, a new solution algorithm that handles frequency dependence of stiffness and damping properties while efficiently computing frequency response functions (FRFs) of complex structures over large frequency ranges is presented. The new solution algorithm in combination with automated multilevel substructuring (AMLS) method is advantageous for solving modally dense frequency response problems (FRPs) with viscoelastic materials (VEMs). In the first section of this chapter, the development of the approach is reviewed and conclusions are drawn from the results discussed in the previous chapter. The last section discusses improvements and possible further work that could be made to enhance the solution approach. 


\subsection{Conclusions}

In Chapter 4, interpolation functions are introduced to reduce the number of finite element (FE) matrices generated to a number much smaller than the number of frequencies at which solutions are needed. We take advantage of the fact that VEM properties vary smoothly with frequency by interpolating between known values to reduce the number of $\mathrm{FE}$ matrices generated, especially if the sampling points are chosen wisely. An approximating subspace is generated on which the interpolated FRP, Eq. (4.10), can be solved efficiently. The key component in decreasing the cost of solving the reduced interpolated FRP is minimizing the dimension of the approximating subspace. Hundreds to thousands of eigenvectors are replaced with a significantly smaller number of enrichment vectors called residual flexibility vectors (RFVs), damping deformation vectors (DDVs), and dynamic response vectors (DRVs). The RFVs represent quasistatic response to loads, the DDVs represent quasistatic response to dashpot forces, and the DRVs represent corrections to approximate solutions of the FRP at select frequencies. The cost of generating the RFVs, DDVs, and DRVs is significantly reduced by computing them on the AMLS subspace $(\mathcal{A})$ and "distilled" subspace $(\mathcal{D})$.

Chapter 5 discusses how the existing Phases 2, 3, 4, and 5 of the software implementation of the AMLS method are modified to handle the additional FE matrices required for the interpolation approach. The main focus of Chapter 5 is on the addition of Phase $4 \mathrm{~d}$ to compute DRVs and Phase $6 \mathrm{~d}$ to solve the reduced 
interpolated FRP. Phase 4d starts with approximating FRP solutions on an initial approximating subspace $\mathcal{W}_{0}$ at several equally spaced frequencies near $\omega_{\max }$ where the approximate solutions are typically inaccurate. DRVs are only computed from residuals computed on the $\mathcal{D}$ subspace whose energy seminorm ratio is above a user supplied cutoff, and for the example FRPs, we set the cutoff to pass the top $50 \%$ of the ratios.

In Chapter 6, we separate the parameters of the solution approach that have a significant impact on solution accuracy and elapsed time into two groups. The parameters of Group One have little to no impact on elapsed time but can determine solution accuracy. The first parameter of Group One is the nominal frequency $\omega_{n}$. For the real-valued frequency independent eigenvalue problem, Eq. $(1.2), \omega_{n}$ is the frequency at which the real part of the frequency-dependent stiffness matrix $\operatorname{Re}\left\{K\left(\omega_{n}\right)\right\}$ is evaluated. We find that FRP solution accuracy increases for increasing values of $\omega_{n}$, so we set $\omega_{n}$ equal to $\omega_{\max }$, where $\omega_{\max }$ is the highest excitation frequency, for all FRPs. The second parameter of Group One is the number of residual flexibility vectors (RFVs). Because the number of RFVs is equal to or less than the number of load cases and the RFVs are inexpensive to compute, we choose to always include RFVs in the approximating subspace. The last parameter of Group One is the number of damping deformation vectors (DDVs). Because the number of DDVs is equal to or less than the number of viscous dampers and the DDVs are inexpensive to compute, we also choose to include DDVs in the approximating subspace. 
Since the parameters of Group Two can have a significant impact on the total elapsed time and solution accuracy, the trade off between accuracy and elapsed time is carefully analyzed. Our approach is to carefully evaluate each parameter in terms of obtaining solutions of similar accuracy to those computed on a much larger approximating subspace containing eigenvectors RFVs, and DDVs. We find that we can set the first parameter of Group Two, the global cutoff frequency $\omega_{g}$, equal to $1.1 \omega_{\max }$ to reduce the dimension of the approximating subspace by an average of $33 \%$. However, reducing $\omega_{g}$ also decreases solution accuracy typically in the frequency range between $0.8 \omega_{\max }$ and $\omega_{\max }$. To increase solution accuracy at a lower cost, we evaluate the second parameter of Group Two, the selection and type of DRVs.

The benefits of using DRVs in place of increasing the global cutoff frequency are carefully evaluated in terms of computational cost. The ratio of the number of DRVs to the number of eigenvectors replaced by DRVs is a good indicator of potential cost savings. These ratios are 1:24, 1:13, and over 1:100 for the cantilever plate, quarter panel, and "body-in-white" FRPs, respectively. As ratio of the number of DRVs to the number of eigenvectors replaced by DRVs increases, the overall percentage of elapsed time saved also increases. This increase in percentage of time saved supports the conclusion that the solution algorithm presented in this dissertation is better suited for modally dense FRPs where we can maximize the ratio of the number of DRVs to the number of eigenvectors replaced by DRVs.

The last parameter of Group Two, the substructure mode cutoff frequency 
$\omega_{A}$, is evaluated in terms of being able to select the approximate solution accuracy we want in trade for elapsed time. First, we find that accurate DRVs can be computed with $\omega_{A}$ equal to $5.0 \omega_{g}$ for the cantilever plate. However, for the quarter panel and "body-in-white" FE models, $\omega_{A}$ needs to be increased to $7.0 \omega_{g}$ to compute accurate DRVs. For the "body-in-white" FE model, we find that we can obtain more accurate solutions in less elapsed time by setting $\omega_{A}$ equal to $9.0 \omega_{g}$ than increasing the global cutoff frequency.

\subsection{Future Work}

Parallelizing the computer implementation of Phases $4 \mathrm{~d}$ and $6 \mathrm{~d}$ for shared memory multiprocessor (SMM) machines with the OpenMP Application Program Interface (API) should be the first step taken. Currently, the sequential implementations of Phase $4 \mathrm{~d}$ and $6 \mathrm{~d}$ take approximately $33 \%$ and $58 \%$ of the total elapsed time for the "body-in-white" FRP, respectively. Based on the parallelization of similar algorithms in the AMLS software implementation [27], a parallel speedup between two and three on 4 processor machine should be expected for Phases $4 \mathrm{~d}$ and $6 \mathrm{~d}$. Any speedup of Phase $4 \mathrm{~d}$ will further increase the overall percentage of elapsed time saved by including DRVs in the approximating subspace, thus making this approach more suitable for FRPs that are not as modally dense.

The majority of the steps of the computer implementation of Phases $4 \mathrm{~d}$ and $6 \mathrm{~d}$ are very also good candidates for parallelization for distributed memory multi- 
processor (DMM) machines that use the Message-Passing Interface (MPI). All of the computationally intensive steps of Phases $4 \mathrm{~d}$ and $6 \mathrm{~d}$ discussed next are good candidates because they require little to no communications between processors. In Phase $4 \mathrm{~d}$, the transformation of the expected ten to twenty $K_{j}$ matrices from the $\mathcal{A}$ to $\mathcal{D}$ and $\mathcal{W}_{0}$ subspaces could be distributed to separate processors. Also in Phase 4d, computing solutions to the FRP on the initial approximating subspace $\mathcal{W}_{0}$ at typically tens of frequencies could also be distributed to separate processors. Computing tens to hundreds of DRVs on the $\mathcal{D}$ subspace is the fourth and computationally most expensive step of Phase $4 \mathrm{~d}$. Presently, the DRVs are computed on the $\mathcal{D}$ subspace by a sequential version of the public domain sparse solver called MUltifrontal Massively Parallel sparse direct Solver (MUMPS). Finally, in Phase 6d, solving the FRP on $\mathcal{W}$ subspace at hundreds of frequencies could easily be distributed to several processors.

Approximating DRVs from the residuals on the $\mathcal{D}$ subspace using just the diagonal of the coefficient matrix resulted in accurate solutions for the simple cantilever plate but not for the practical FE models. A robust iterative approach for inexpensively approximating DRVs may result in a larger number of less accurate DRVs. However, any approach that increases the dimension of the approximating subspace should be carefully tested to ensure the cost savings in creating inexpensive DRVs are not lost in computing approximate solutions on a larger approximating subspace.

Replacing thousands of eigenvectors with tens to hundreds of DRVs could 
prove to be very beneficial in reducing the cost of solving a large class of FRPs that do not have frequency dependent material properties. There is a factorization or eigensolution cost that is proportional to the cube of the subspace dimension in the frequency independent non-proportional (non-modal) damping case that could be reduced by including DRVs in the approximating subspace. For example, if the "body-in-white" automobile body FRP presented in Ch. 6 did not have frequency dependent materials, similar reductions in the dimension of approximating subspace could be realized.

Because this research is a proof-of-concept effort, the number and location of DRV frequencies and the user-supplied cutoff were chosen arbitrarily. We showed that significant cost savings can be realized by reducing the dimension of the eigenspace and enriching the approximating subspace with carefully chosen vectors. However, in order for this solution approach to be developed to the level required for practical industrial use, a robust and efficient process of automatically selecting the number and location of DRV frequencies would need to be developed. For example, an adaptive approach could be developed to maximize the probability that we would only compute the DRVs that would do the most to improve accuracy. In this approach, we would compute DRVs in several iterations. In each iteration we would only compute the few DRVs that made the largest contribution to the accuracy of the solution for that iteration. At the end of each iteration, we would include those DRVs in the initial approximating subspace. Through this process, we would incrementally improve solution accuracy and minimize the chances of com- 
puting unnecessary DRVs. This adaptive approach would require that we change the overall structure of the code because the initial approximating subspace $\mathcal{W}_{0}$ would grow in dimension as DRVs are incrementally computed and added into the approximating subspace. Additionally, an industrial strength implementation of this solution approach would also need to have the capability to automatically handle large numbers of load cases, viscous dampers, and VEMs. 


\section{Bibliography}

[1] Adhikari, S., "Damping Models for Structural Vibration", Ph.D. Dissertation, Trinity College, Cambridge, England, 2000.

[2] Agnes, G. S., "Towards Single-element Modeling of Multi-layer Constrained Layer Damping Treatments", Proceedings of the SPIE - The International Society of Optical Engineering, vol 2445, Bellingham, WA, pp 336-347, May 1995.

[3] Akanda, A. and Goetchius, G. M., "Representation of Constrained/Unconstrained Layer Damping Treatments in FEA/SEA Vehicle System Models: A Simplified Approach", Proceedings of the 1999 Noise and Vibration Conference, Traverse City, MI, pp. 342-355, 1999.

[4] Balmès, E., "Model Reduction for Systems with Frequency Dependent Properties", International Modal Analysis Conference, Dearborn, Michigan, 1997.

[5] Balmès, E., "Parametric Families of Reduced Finite Element Models. Theory and Applications", Mechanical Systems and Signal Processing, Vol. 10, No. 4, pp. 381-394, 1996. 
[6] Balmès, E.,p Structural Dynamics Toolbox for MATLAB, http://www.sdtools.com, 2005.

[7] Balmès, E., "Superelement Representation of a Model with Frequency Dependent Properties", Int. Seminar on Modal Analysis, Leuven, pp. 1767-1778, 1996.

[8] Balmès E. and Germès, S., "Design Strategies for Viscoelastic Damping Treatment Applied to Automotive Components", International Modal Analysis Conference, Dearborn, Michigan, 2004.

[9] Balmès, E. and Germès, S., "Tools for Viscoelastic Damping Treatment Design. Application to an Automotive Floor Panel", International Seminar on Modal Analysis, Leuven, 2002.

[10] Barrett, R., Berry, M., Chan, T. F., Demmel, J., Donato, J. M., Dongarra, J., Eijkhout, V., Pozo, R., Romine, C., and Van der Vorst, H., Templates for the Solution of Linear Systems: Building Blocks for Iterative Methods, Second Ed., SIAM, Philadelphia, Pennsylvania,1994.

[11] Bennighof, J. K., "Adaptive Multi-Level Substructuring Method for Acoustic Radiation and Scattering from Complex Structures", Computational Methods for Fluid/Structure Interaction, edited by A. J. Kalinowski, vol 178 ASME, New York, pp. 25-38, 1993.

[12] Bennighof, J. K. and Kaplan, M. F., "Frequency Window Implementation of 
Adaptive Multi-level Substructuring", Journal of Vibration and Acoustics, Vol 120, No. 2, pp. 409-418, 1998.

[13] Bennighof, J. K. and Kaplan, M. F., "Frequency Sweep Analysis Using MultiLevel Substructuring, Global Modes, and Iteration", Proceedings of the $39^{\text {th }}$ AIAA/ASME/ASCE/AHS Structures, Structural Dynamics and Materials Conference, Long Beach, Paper No. 98-2015, 1998.

[14] Bennighof, J. K., Kaplan, M. F., and Muller, M. B., "Extending the Frequency Response Capabilities of Adaptive Multi-level Substructuring", Proceedings of the $41^{\text {st }}$ SDM Conference, Atlanta, Georgia, 2000.

[15] Bennighof, J. K., Kaplan M. F., Muller, M. B., and Kim, M., "Meeting the NVH Computational Challenge: Adaptive Multi-level Substructuring", Proceedings of the $18^{\text {th }}$ International Modal Analysis Conference, San Antonio, Texas, 2000.

[16] Bennighof, J. K., Kaplan, M. F., Muller, M. B., and Petesch, D. J., "Optimizing Noise and Vibration Performance Using Automated Multi-Level Substructuring", Proceedings of the $32^{\text {nd }}$ International Symposium on Automotive Technology and Automation, Vienna, Austria, 1999.

[17] Bennighof, J. K. and Kim, C. K., "An Adaptive Multi-Level Substructuring Method for Efficient Modelling of Complex Structures", Proceedings of the AIAA 33rd SDM Conference, Dallas, Texas, 1992.

[18] Beranek, L. L. (Editor) and Vér, I. L. (Editor), Noise and Vibration Control 
Engineering: Principles and Applications, New York: John Wiley and Sons, 1992.

[19] Björck, A., "Numerics of Gram-Schmidt Orthogonalization", Linear Algebra and Its Applications, vol 197, pp. 297-316, 1994.

[20] Bloemhof, H., "Optimization of Damping Treatment in the Car Design Process Using Advanced Simulation Techniques", Proceedings of the 1995 Noise and Vibration Conference, Traverse City, MI, pp 249, 1995.

[21] Craig, R. R. Jr., Structural Dynamics: An Introduction to Computer Methods, New York: John Wiley and Sons, 1981.

[22] Craig, R. R. Jr. and Bampton, M. C., "Coupling Substructures for Dynamic Analysis", AIAA Journal, vol. 6, no. 7, pp 1313-1319, 1968.

[23] Gibson, W. C. and McTavish, D. J., "Implementation of the GHM Method for Viscoelastic Materials using MATLAB and NASTRAN", Proceedings of the SPIE - The International Society of Optical Engineering, vol 2445, Bellingham, WA, pp 312-323, 1995.

[24] Johnson, C. D. and Kienholz, D. A., "Finite Element Predictions of Damping in Structures with Constrained Viscoelastic Layers ", AIAA Journal, vol. 20, no. 9, pp 1284-1290, 1982.

[25] Kaplan, M. F., "Implementation of Automated Multilevel Substructuring for 
Frequency Response Analysis of Structures", Ph.D. Dissertation, University of Texas at Austin, Texas, 2001.

[26] Kim, C. W., “ Frequency Response Analysis of a Structure with Damping and Acoustic Fluid Automated Multilevel Substructuring ", Ph.D. Dissertation, University of Texas at Austin, Texas, 2004.

[27] Kim, M., "An Efficient Eigensolution Method and Its Implementation for Large Structural Systems", Ph.D. Dissertation, University of Texas at Austin, Texas, 2004.

[28] Leo, D. J., Austin, E. M., and Beattie, C., "Constrained Substructure Approach to Optimal Strain Energy", Transactions of the ASME, vol. 123, pp 340-346, 2001.

[29] Macioce, P., "Viscoelastic Damping 101", www.roushind.com/news_downloads /white_papers/SV_Damping101.pdf, Roush Industries, Inc.

[30] McTavish, D. J., Hughes, P. C., Soucy, Y., and Graham, W. B., "Prediction and Measurement of Modeling Damping Factors for Viscoelastic Space Structures", AIAA Journal, Vol. 30, No. 5, 1992.

[31] Meirovitch, L., Elements of Vibration Analysis, 2d ed., McGraw-Hill, Inc. New York, New York, 1986.

[32] Mignery, A. L., "Quiet Steel ${ }^{\circledR}$ Body Panel Design with DAMP ${ }^{\circledR}$ - A Custom 
Preprocessor Utilizing MSC-Patran/Nastran”, Material Sciences Corporation, Laminates and Composites, Technical Report, Elk Grove Village, IL.

[33] Mignery, A. L. and Vydra, E. J., "Vibration Analysis of Metal/Polymer/Metal Laminates - Approximate Versus Viscoelastic methods", SAE Noise and Vibration Conference, Traverse City, MI, pp. 579-590, 1997.

[34] Nashif, A. D., Jones, D. I. G., and Henderson, J. P., Vibration Damping, New York: John Wiley and Sons, 1985.

[35] Park, C. H., Inman, D. J., and Lam, M. J., "Model Reduction of Viscoelastic Finite Elements", Journal of Sound and Vibration, vol 219, no 4, pp 619-637, 1999.

[36] Plouin, A. and Balmès, E., "Steel/Viscoelastic/Steel Shells Computational Methods and Experimental Validations", International Modal Analysis Conference, San Antonio, Texas, 2000.

[37] Rao, M. D., "Recent Applications of Viscoelastic Damping for Noise Control in Automobiles and Commercial Airplanes", Journal of Sound and Vibration, vol 262, pp. 457-474, 2003.

[38] Ross, D., Ungar, E. E., and Kerwin, E. M., "Damping of Plate Flexural Vibrations by Means of Viscoelastic Laminae", in Ruzicka (ed.), Structural Damping, ASME, New York, Sec. 3, 1959. 
[39] Rusovici, R. and Lesieutre, G. A., "Investigations of Viscoelastic Structure Behavior Using a Three-Dimensional Anelastic Displacement Finite Element", Smart Structures and Materials 2002: Damping and Isolation, vol 5052, pp 151$162,2003$.

[40] Sun, C. T. and Lu, Y., Vibration Damping of Structural Elements, PrenticeHall, Inc. Englewood Cliffs, New Jersey, 1995.

[41] Xu, Y., Lu, Y., and Wang, B., "Revised Modal Strain Energy Method for Finite Element Analysis of Viscoelastic Damping Treated Structures", Smart Structures and Materials 2002: Damping and Isolation, vol 4697, pp 35-42, 2002. 


\section{Vita}

Eric D. Swenson was born on April 11, 1967 in Iowa City, Iowa. He enlisted in the United States Air Force in July 1985. After completing a year-long Systems Repair Technician technical school at Lowry AFB, CO, Airman Swenson worked at the McClellan Central Labs at McClellan AFB, CA as an electronics technician. Three

years later, Sergeant Swenson was selected for the Airmen Education Commissioning Program. He went on to earn a bachelors of science from the Ohio State University in Columbus, Ohio where he graduated Magna Cum Laude with Honors in Civil Engineering in March 1993. He went to Officer Training School at Lackland AFB where he graduated as a Distinguished Graduate in July 1993.

After commissioning, Second Lieutenant Swenson was stationed at Holloman AFB, NM where he served as a civil engineer in the 49th Civil Engineer Squadron. In 1996, First Lieutenant was selected to attend the Air Force Institute of Technology (AFIT) Graduate School of Engineering to earn a masters of science degree in astronautical engineering. After graduation, he moved to Patrick AFB, FL and worked as a Titan Launch Program project engineer at Cape Canveral Air Force 
Station, FL.

After serving a two year tour at Incirlik AB, Turkey, he started a Ph.D. program at the University of Texas, Austin, TX. Major Eric Swenson married his high school sweetheart Kristine Anne Leslie in 1985 and has two sons Sean and Seth and a daughter Sydney.

Permanent Address: 3685 Meadowcourt Dr

Beavercreek, OH 45431

This dissertation was typeset with $\mathrm{LT}_{\mathrm{E}} \mathrm{X} 2 \varepsilon^{1}$ by the author.

\footnotetext{
${ }^{1} \mathrm{~L}^{\mathrm{A}} \mathrm{T}_{\mathrm{E}} \mathrm{X} 2 \varepsilon$ is an extension of $\mathrm{L}^{\mathrm{A}} \mathrm{T}_{\mathrm{E}} \mathrm{X}$. ${ }^{\mathrm{A}} \mathrm{T} \mathrm{E} \mathrm{X}$ is a collection of macros for $\mathrm{T}_{\mathrm{E}} \mathrm{X}$. $\mathrm{T}_{\mathrm{E}} \mathrm{X}$ is a trademark of the American Mathematical Society. The macros used in formatting this dissertation were written by Dinesh Das, Department of Computer Sciences, The University of Texas at Austin.
} 